\title{
Comprimentos de álgebras não associativas
}

\author{
Carlos André Gomes Silva \\ DISSERTAÇÃO APRESENTADA \\ AO \\ INSTITUTO DE MATEMÁTICA E ESTATÍSTICA \\ DA \\ Universidade de SÃo PaUlo \\ PARA \\ OBTENÇÃO DO TÍTULO \\ DE \\ MESTRE EM CIÊNCIAS \\ Programa: Matemática \\ Orientador: Prof. Dr. Rodrigo Lucas Rodrigues
}

Durante o desenvolvimento deste trabalho o autor recebeu auxílio financeiro do CNPq.

São Paulo, Agosto de 2020. 


\section{Comprimentos de álgebras não associativas}

Esta versão da dissertação contém as correções e alterações sugeridas pela Comissão Julgadora durante a defesa da versão original do trabalho, realizada por Carlos André Gomes Silva em 31/07/2020. Uma cópia da versão original está disponível no Instituto de Matemática e Estatística da Universidade de São Paulo.

Comissão Julgadora:

- Prof. Dr. Rodrigo Lucas Rodrigues - Universidade Federal do Ceará - UFC

- Profa. Dra. Ma. Isabel Hernández - Centro de Investigación en Matemáticas CONACYT, A.C. CIMAT

- Prof. Dr. Victor Hugo López Solís - Universidad Nacional Santiago Antunez de Mayolo - UNASAM 


\title{
Agradecimentos
}

\author{
Agradeço a Deus pela existência.
}

Aos meus pais, Maria Edileuza e Manoel Arnobio pelos ensinamentos e apoio durante a minha vida e aos meus irmãos Ted, Sebastião e Cícero por todo o suporte oferecido durante meu percurso no mestrado.

Ao meu orientador prof. Rodrigo Lucas Rodrigues pela oportunidade, sugestões e paciência que foram fundamentais para a conclusão deste trabalho e que me tornaram um profissional melhor.

Aos professores Dra. Ma. Isabel Hernández e Dr. Victor Hugo López Solís pelas valiosas correções, recomendações e por terem dedicado parte do seu tempo para a leitura deste trabalho.

Agradeço à minha namorada Manuela Joaquina pela parceria, compreensão e por me mostrar a importância da construção de um trabalho sólido.

Aos meus colegas de departamento e amigos que conheci durante meu percurso e que contribuíram efetivamente para a minha formação; Maria Christina, Ítalo, Elizabeth, Rafael, Alexandre, Alan, Allen, Gabriel Magalhães, Delvânia e Lara.

Ao CNPq pelo auxílio financeiro. 


\section{Resumo}

Silva, C. A. G. Comprimentos de álgebras não associativas. 2020. Dissertação (Mestrado) - Instituto de Matemática e Estatística, Universidade de São Paulo, São Paulo, 2020.

Nesta dissertação estudamos o comprimento de álgebras de dimensão finita. Estabelecemos limites superiores para seus comprimentos e exibimos propriedades construtivas dos conjuntos geradores para álgebras arbitrárias em dimensões inferiores a 5. Além disso, fornecemos uma classificação das álgebras alternativas de dimensão inferior a 6 sobre corpos perfeitos baseada em seus comprimentos.

Palavras-chave: álgebra, cadeia, comprimento, conjunto gerador, dimensão, palavra irredutível. 


\section{Abstract}

Silva, C. A. G. Lengths of non-associative algebras. 2020. Dissertação (Mestrado) - Instituto de Matemática e Estatísitca - Universidade de São Paulo, São Paulo, 2020.

In this work we study the length of finite-dimensional algebras. We establish upper bounds to their lengths and exhibit constructive properties of generating sets for arbitrary algebras of dimension smaller than 5. Furthermore a complete classification up to isomorphism is given to alternative algebras of dimension smaller than 6 over perfect fields based on its length.

Keywords: algebra, chain, length, generating set, dimension, irreducible word. 


\section{Sumário}

$\begin{array}{ll}\text { Lista de símbolos e abreviaturas } & \text { ix }\end{array}$

Introdução

1 Preliminares 1

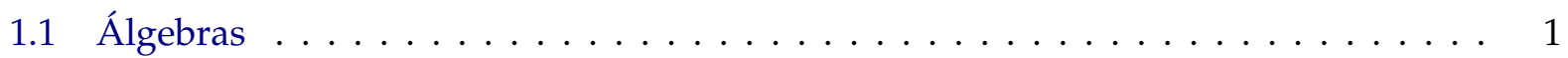

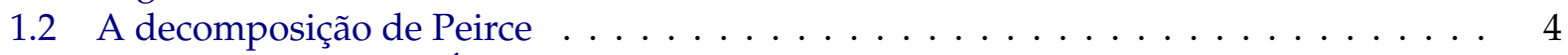

1.3 Homomorfismos de Álgebras . . . . . . . . . . . . . . . . . . . . 6

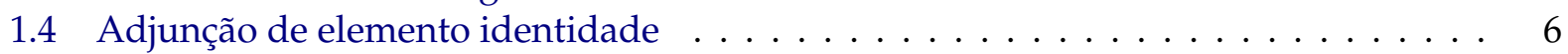

1.5 O processo de duplicação de Cayley-Dickson . . . . . . . . . . . . . . . . 7

2 O Comprimento de uma Álgebra 9

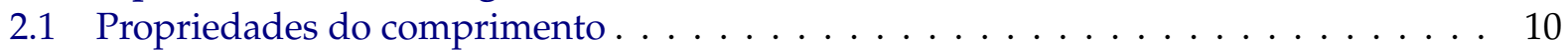

2.2 Palavras Irredutíveis . . . . . . . . . . . . . . . . . . . . . 14

2.2.1 Sequências características e suas propriedades básicas . . . . . . . . . . . . 15

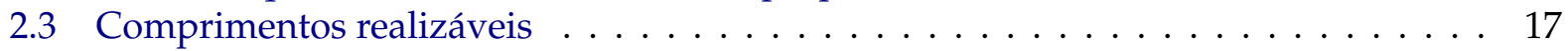

3 O comprimento de álgebras de dimensão baixa $\quad 21$

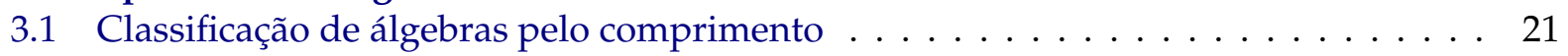

3.1 .1 Nota histórica . . . . . . . . . . . . . . . . . . . . . 21

3.1 .2 Caracterizações parciais . . . . . . . . . . . . . . . . 22

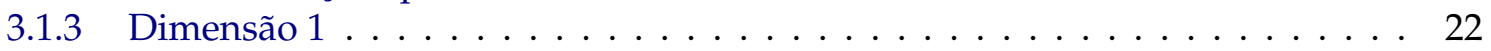

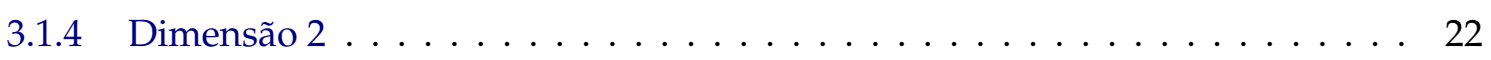

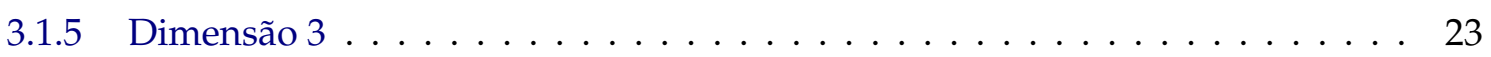

3.1 .6 Dimensão $4 \ldots \ldots \ldots \ldots \ldots \ldots \ldots \ldots \ldots \ldots$

4 O comprimento em Álgebras Associativas 31

4.1 A conjectura de Paz . . . . . . . . . . . . . . . . . . 31

4.2 Resultados Complementares . . . . . . . . . . . . . . . . . . . . . 32

4.3 Álgebras associativas de dimensões menores ou iguais a $4 \ldots \ldots \ldots$. . . . . . . . . 34

5 O comprimento em Álgebras Alternativas 37

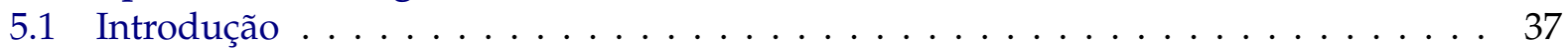

5.2 Resultados preliminares . . . . . . . . . . . . . . . . . . . 37

5.3 Classificação das álgebras de dimensão menor que $6 \ldots \ldots \ldots \ldots$. . . . . . . . . . . . . . . .

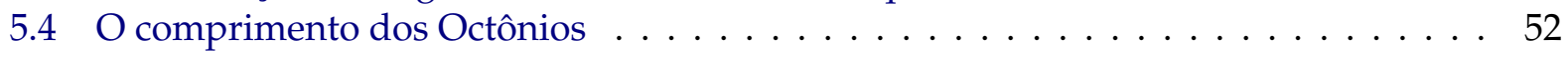

A Programas Auxiliares em linguagem C $\quad 59$

$\begin{array}{ll}\text { Referências Bibliográficas } & 67\end{array}$ 


\title{
Lista de símbolos e abreviaturas
}

\author{
$\mathbb{R} \quad$ Conjunto dos números reais \\ C Conjunto dos números complexos \\ $M_{n}(K) \quad$ Conjunto das matrizes quadradas de ordem $n$ com entradas no corpo $K$ \\ $\langle A\rangle \quad$ Espaço vetorial gerado pelo conjunto $A$ \\ $(x, y, z) \quad$ Associador dos elementos $x, y, z$, isto é, $(x y) z-x(y z)$ \\ $K x \quad$ Conjunto formado pelos elementos da forma $k . x \operatorname{com} k \in K$ \\ $\mathbb{Z}_{2} \quad$ Corpo com dois elementos \\ $K\left[x_{1}, \cdots, x_{n}\right]$ Anel dos polinômios nas variáveis $x_{1}, \cdots, x_{n}$ com coeficientes no corpo $K$.
}




\section{Introdução}

Comprimentos de álgebras é um ramo da álgebra iniciado em 1958 quando Rivlin e Spencer [25] utilizaram pela primeira vez a teoria para estudar polinômios de matrizes de ordem três e aplicaram esses resultados em mecânica de meios contínuos.

Alguns anos mais tarde, Paz [20] estabeleceu a linguagem que seria adotada e estendeu as conclusões publicadas por Rivlin e Spencer para álgebras de matrizes de ordem arbitrária. Paz foi também pioneiro no estudo do problema de limitar o comprimento da álgebra de matrizes em função da ordem, estabelecendo uma cota superior e conjecturando um resultado que seria conhecido como A Conjectura de Paz. Desde então o problema de determinar a menor cota superior para o comprimento das álgebras de matrizes tem sido um campo ativo de pesquisa.

Os trabalhos de Markova e Guterman [8],[14] contribuíram substancialmente para o desenvolvimento da teoria de comprimentos de álgebras associativas e podem ser analisados sob duas óticas; estendem os resultados obtidos por Paz para álgebras associativas, caracterizando os casos de comprimentos maximais e minimais e realizam avanços em direção ao estabelecimento de um limite superior para o comprimento de álgebras associativas.

Um outro direcionamento que consiste em fixar uma classe de álgebras e então limitar seu comprimento em função da dimensão ganhou notoriedade com os trabalhos de Guterman e Kudryavtsev [7] que definiram limites superiores para o comprimento de álgebras arbitrárias.

Dedicamos este trabalho ao estudo do comprimento de álgebras não associativas, isto é, a identidade $(x y) z=x(y z)$ não é necessariamente verdadeira, além disso as álgebras terão sempre dimensão finita. Para casos em que a dimensão é inferior a 5, abordamos o problema de determinar os comprimentos atingidos por álgebras alternativas. Revisitamos problemas na determinação do comprimento de álgebras arbitrárias e obtemos novos resultados através da adição de hipóteses como associatividade e alternatividade, sendo possível determinar o comprimento de classes de isomorfismos de álgebras.

A dissertação está dividida em cinco capítulos e um apêndice. No primeiro capítulo introduzimos noções básicas como a de álgebra e a de homomorfismo. Definimos as diversas propriedades que uma álgebra pode assumir e que serão tratadas no decorrer da dissertação. Introduzimos ainda algumas ferramentas como a adjunção de elemento identidade e a decomposição de Peirce, que serão utilizadas nos demais capítulos.

Inserimos no segundo capítulo a linguagem de comprimentos de álgebras, concatenando definições e exemplos para facilitar a compreensão e introduzimos o conceito de cadeia e de refinamento. Exibimos propriedades que serão utilizadas nos demais capítulos e fornecemos aplicações para ilustrar suas consequências.

Dedicamos o Capítulo 3 ao estudo de álgebras de comprimento inferior a 5 e estabelecemos quatro teoremas que versam sobre as propriedades dos conjuntos que realizam o comprimento da álgebra, tais teoremas se mostram fundamentais pois permitem reduzir o limitante superior 
para o comprimento de uma álgebra, como feito no Capítulo 4. A demonstração do teorema se dá de forma construtiva, isto é, supomos que uma álgebra tenha as propriedades dadas e mostramos que estas induzem propriedades nos conjuntos geradores. Quando estas propriedades forem necessárias mas não suficientes, apresentamos contraexemplos.

O Capítulo 4 é dedicado à avaliação do comprimento de álgebras associativas; e verificamos como o comprimento se relaciona com os homomorfismos sobrejetores. Determinamos ainda um limitante linear trivial para o comprimento em função da dimensão, refinamos os resultados apresentados no Capítulo 3 utilizando a associatividade e analisamos o comportamento do comprimento em álgebras associativas que são comutativas, em particular, determinamos condições necessárias e suficientes para que uma álgebra de matrizes que é comutativa tenha comprimento maximal.

Durante o Capítulo 5 nos concentramos nas álgebras alternativas que não são associativas. Após uma breve introdução com resultados preliminares como o Teorema de Artin e as identidades de Moufang, nos apoiamos no trabalho de Zhou e Goodaire [6] sobre álgebras alternativas para estudar classes de isomorfismos de álgebras alternativas em dimensão inferior a 6 . Dividimos o cálculo do comprimento em três etapas; limitar superiormente o comprimento de uma álgebra alternativa arbitrária por 3, caracterizar as bases originadas por conjuntos geradores de comprimento 3 e estudar as tabelas multiplicativas para identificar quais álgebras podem atingilos. Por fim, demonstramos que quando $K$ é um corpo perfeito, as $K$-álgebras alternativas que não são associativas de dimensão inferior a 6 possuem comprimento 2.

Finalmente, no Apêndice A fornecemos um algoritmo em linguagem $C$ que pode auxiliar na avaliação de produtos de elementos nas álgebras alternativas determinadas no Capítulo 5, bem como o processo de criação destes algoritmos. 


\section{Capítulo 1}

\section{Preliminares}

\section{1 Álgebras}

Sucintamente uma álgebra $A$ sobre um corpo $K$ é um $K$-espaço vetorial com uma operação bilinear também chamada de produto. Vamos agora introduzir algumas definições e propriedades fundamentais para o desenvolvimento do arcabouço teórico do trabalho, começando pela:

Definição 1.1 (Álgebra). Sejam $K$ um corpo e $A$ um $K$-espaço vetorial munido de uma operação binária, usualmente chamada de multiplicação, $f: A \times A \rightarrow A$. Dizemos que $A$ é uma $K$-álgebra se, para quaisquer elementos $x, y, z \in A$ e $a \in K$, as seguintes igualdades são verdadeiras:

- $f(x+y, z)=f(x, z)+f(y, z)$

- $f(z, x+y)=f(z, x)+f(z, y)$

- $f(a x, y)=a f(x, y)=f(x, a y)$

Isto é, se a função $f$ é bilinear.

Iremos denotar a imagem do par $(x, y)$ pela função $f$ apenas por $x y$ sempre que a função $f$ estiver clara pelo contexto. A seguir, vejamos alguns exemplos clássicos de álgebras.

Exemplo 1.1. O espaço vetorial real $\mathbb{R}$ de dimensão 1 munido da operação * que denota o produto usual entre dois números reais forma uma álgebra.

Exemplo 1.2. Dado um $K$-espaço vetorial $V$, o espaço vetorial formado por todos os endomorfismos de $V$ munido da operação de composição de funções forma uma álgebra.

Definição 1.2 (Dependência e Independência linear). Se $S=\left\{v_{i}: i \in I\right\}$ é um subconjunto de um $K$ espaço vetorial, dizemos que os vetores de $S$ são linearmente dependentes se existir uma família finita de escalares não nulos $\left\{a_{j}: j \in J \subset I\right\}$ tal que $\sum_{j \in J} a_{j} v_{j}=0$. Caso contrário, os vetores de $S$ são ditos linearmente independentes.

Exemplo 1.3. Os subconjuntos $S=\left\{e_{1}, \ldots, e_{n}\right\}$ e $S^{\prime}=\left\{e_{1}, e_{2}\right\}$ de vetores da base canônica de $\mathbb{R}^{n}$ são linearmente independentes enquanto o conjunto $S^{\prime \prime}=\left\{e_{1}, e_{2}, e_{1}+e_{2}\right\}$ é linearmente dependente. A principal diferença entre $S$ e $S^{\prime}$ é que apenas um deles gera o $\mathbb{R}$-espaço vetorial $\mathbb{R}^{n}$, o que pode ser melhor entendido na próxima definição. 
Definição 1.3 (Base). Dado um $K$-espaço vetorial $V$, dizemos que um subconjunto $\mathcal{B} \subset V$ é uma base para $V$ se os vetores de $\mathcal{B}$ são linearmente independentes e se este gera o espaço vetorial $V$. Analogamente, se $A$ é uma $K$-álgebra, um conjunto $\mathcal{B}$ é dito uma base de $A$ se os vetores de $\mathcal{B}$ são linearmente independentes e se $\mathcal{B}$ gera a álgebra $A$. A dimensão da álgebra é a dimensão desta vista como K-espaço vetorial.

Definição 1.4 (Álgebra Oposta). Se $A$ é uma $K$-álgebra com multiplicação $f$ então o espaço vetorial $A$ munido da operação $f_{o p}: A \times A \rightarrow A$ definida por $f_{o p}(x, y)=f(y, x)$ também forma uma álgebra, chamada de álgebra oposta e denotada por $A^{o p}$.

Definição 1.5 (Subálgebra). Um subespaço vetorial $S$ de uma álgebra $A$ munido de uma multiplicação $f$ que é fechado para a multiplicação $f$ restrita a $S \times S$ será chamado de subálgebra de A.

Definição 1.6 (Ideal de uma Álgebra). Um subespaço vetorial $I$ de uma álgebra $A$ que satisfaz $(x a) \in I$ para quaisquer $a \in A$ e $x \in I$ será chamado ideal à direita. Analogamente $I$ será chamado ideal à esquerda se satisfizer $(a x) \in I$ para quaisquer $a \in A$ e $x \in I$.

Assim como no contexto de anéis podemos atribuir regularidade às álgebras através de identidades que a operação produto possa satisfazer. A seguir definiremos as classes de álgebras que aparecem nesta dissertação.

Definição 1.7. Dada uma $K$-álgebra $A$, dizemos que

1. A é unitária se possui um elemento identidade multiplicativo, o qual será denotado por $1_{A}$;

2. A é comutativa se satisfaz $x y=y x$ para quaisquer $x, y \in A$;

3. $A$ é associativa se $(x y) z=x(y z)$ para quaisquer $x, y, z \in A$;

4. $A$ é alternativa se $(x x) y=x(x y)$ e $(y x) x=y(x x)$ para quaisquer $x, y \in A$;

5. A é flexível se $(x y) x=x(y x)$ para quaisquer $x, y \in A$;

6. A é chamada álgebra de potências associativas se toda subálgebra gerada por um elemento qualquer é associativa;

7. A é chamada álgebra de Jordan se é comutativa e se satisfaz a identidade de Jordan $(x y)(x x)=$ $x(y(x x))$.

Segue das definições apresentadas que qualquer álgebra comutativa é flexível e que associatividade e alternatividade implicam na associatividade de potências. A seguir ilustramos como algumas destas álgebras se relacionam quando são unitárias e estão definidas sobre um corpo de característica diferente de 2 .

$$
\text { Associatividade } \Rightarrow\left\{\begin{array}{l}
\text { Identidade de Jordan } \Rightarrow \text { Flexibilidade } \\
\text { Alternatividade } \Rightarrow\left\{\begin{array}{l}
\text { Flexibilidade } \\
\text { Associatividade de potências }
\end{array}\right. \\
\text { Associatividade de potências }
\end{array}\right.
$$




\section{Comutatividade $\Rightarrow$ Flexibilidade}

Podemos utilizar os conceitos acima para derivar outras propriedades para os elementos de uma álgebra e assim definir novas classes de álgebras através dessas propriedades. Fixamos um elemento $x$ de uma $K$-álgebra $A$ que é de potências associativas e definimos recursivamente as potências de $x$ por $x^{1}=x$ e $x^{i+1}=x x^{i}$, para $i=1,2, \ldots$ e desse modo as potências de um elemento qualquer estão bem definidas e então podemos introduzir a seguinte definição.

Definição 1.8. Sejam $A$ uma $K$-álgebra de potências associativas e $a \in A$. O elemento $a$ é chamado de nilpotente se existe um inteiro positivo $n$ tal que $a^{n}=0$. Quando todos os elementos de uma álgebra (resp. ideal) são nilpotentes, denominamos esta álgebra de Nilálgebra (resp. Nilideal).

Uma Zero álgebra é uma álgebra cujo produto de quaisquer dois elementos é nulo, naturalmente qualquer zero álgebra é uma nilálgebra. Toda álgebra de potências associativas $A$ possui um nilideal maximal $R(A)$ tal que o quociente $A / R(A)$ não contém nilpotentes não nulos, portanto não contém nilideais não nulos. Este ideal é chamado de Radical de $A$.

Definição 1.9 (Álgebra Semissimples). Sejam $A$ uma $K$-álgebra e $R(A)$ seu radical, dizemos que $A$ é semissimples se $R(A)=0$.

Observe que pelo que foi feito acima, se $A$ é uma $K$-álgebra alternativa e $R(A)$ é seu radical, então o radical de $A / R(A)$ é nulo, isto é, $A / R(A)$ é semissimples.

Definição 1.10 (Álgebra Separável). Uma $K$-álgebra é separável se para toda extensão $K^{\prime}$ do corpo $K$ a álgebra $A \otimes_{k} K^{\prime}$ é semissimples.

Definição 1.11 (Corpo Perfeito). Seja $K$ um corpo, dizemos que $K$ é perfeito se toda extensão finita de $K$ é separável.

Exemplo 1.4. $\mathrm{O} \mathbb{R}$-espaço vetorial $M_{n}(\mathbb{R})$ munido do produto usual entre matrizes forma uma álgebra associativa. Este é um caso particular do Exemplo 1.2 tomando $V=\mathbb{R}^{n}$. De modo geral, o K-espaço vetorial $M_{n}(K)$ munido do produto usual entre matrizes forma uma álgebra que é associativa mas, em geral, não é comutativa. Observe que utilizando o Exemplo 1.2 e fazendo corresponder a cada matriz um operador linear, provar a associatividade do produto se resume a provar a associatividade da composição de operadores.

Exemplo 1.5. Considere a $\mathbb{R}$-álgebra $A$ com base $\left\{e_{1}, e_{2}\right\}$ e tábua:

\begin{tabular}{||l|l|l|}
\hline$*$ & $e_{1}$ & $e_{2}$ \\
\hline$e_{1}$ & 0 & $e_{2}$ \\
\hline$e_{2}$ & $e_{2}$ & 0 \\
\hline
\end{tabular}

Neste caso, $A$ é uma álgebra comutativa, no entanto como $\left(e_{1}+e_{2}\right)^{2} e_{2} \neq\left(e_{1}+e_{2}\right)\left(\left(e_{1}+e_{2}\right) e_{2}\right)$ concluímos que $A$ não é associativa nem alternativa. 


\subsection{A decomposição de Peirce}

Durante esta seção iremos apresentar um método de decomposição de uma álgebra alternativa $A$ em uma soma de subespaços vetoriais. Este método será particularmente útil no Capítulo 5 onde será usado na classificação das álgebras alternativas.

Um elemento $x$ de uma álgebra $A$ é chamado de idempotente se satisfaz a igualdade $x^{2}=x$, é imediato que os elementos $1_{A}$ e 0 são idempotentes, e tais elementos são chamados de idempotentes triviais de $A$. Para o desenvolvimento do método em questão estamos interessados em idempotentes não triviais. A existência de idempotentes não triviais em uma álgebra alternativa que não é uma nilálgebra é garantida pelo lema a seguir:

Lema 1.1. [22] Qualquer álgebra de dimensão finita que é de potências associativas e que não é uma nilálgebra, contém um idempotente $e \neq 0$.

Passamos para o caso em que $A$ é uma $K$-álgebra alternativa, unitária e que não é nilálgebra com um idempotente não trivial denotado por $e$. Consideramos as funções $L_{e}: A \rightarrow A$ definida por $L_{e}(x)=$ ex e $R_{e}: A \rightarrow A$ definida por $R_{e}(x)=x e$ conhecidas como operador de multiplicação à esquerda e operador de multiplicação à direita, respectivamente.

Primeiramente, vamos mostrar que as funções definidas acima são lineares, fazendo isso para $R_{e}$ e de modo análogo o resultado será válido para $L_{e}$. Com efeito, dados $x, y \in A$ e $k \in K$, $R_{e}(x+y)=(x+y) e=x e+y e=R_{e}(x)+R_{e}(y)$ e $R_{e}(k x)=(k x) e=k(x e)=k R_{e}(x)$. Desse modo $R_{e} \mathrm{e}$ $L_{e}$ são homomorfismos de espaços vetoriais. Considere os conjuntos:

- O conjunto $A_{11}$ satisfazendo $\left\{\begin{array}{l}\left.L_{e}\right|_{A_{11}}=I d \\ \left.R_{e}\right|_{A_{11}}=I d\end{array}\right.$

- O conjunto $A_{10}$ satisfazendo $\left\{\begin{array}{l}\left.L_{e}\right|_{A_{10}}=I d \\ \left.R_{e}\right|_{A_{10}}=0\end{array}\right.$

- O conjunto $A_{01}$ satisfazendo $\left\{\begin{array}{l}\left.L_{e}\right|_{A_{01}}=0 \\ \left.R_{e}\right|_{A_{01}}=I d\end{array}\right.$

- O conjunto $A_{00}$ satisfazendo $\left\{\begin{array}{l}\left.L_{e}\right|_{A_{00}}=0 \\ \left.R_{e}\right|_{A_{00}}=0\end{array}\right.$

Afirmamos que o $K$-espaço vetorial $A$ pode ser expresso pela soma $A=A_{11}+A_{10}+A_{01}+A_{00}$, onde cada uma destas parcelas é um subespaço vetorial satisfazendo as respectivas condições apresentadas. De fato, dado $x \in A$, podemos decompô-lo em $x=e x e+(e x-e x e)+(x e-e x e)+(x-e x-x e+$ exe). Para o que falta, considere as seguintes igualdades:

$$
\left\{\begin{array}{l}
R_{e}(\text { exe })=e x e^{2}=\text { exe } \\
L_{e}(\text { exe })=e^{2} x e=\text { exe }
\end{array}\right.
$$




$$
\begin{aligned}
& \left\{\begin{array}{l}
R_{e}(e x-e x e)=(e x-e x e) e=(e x) e-(e x e) e=e x e-e x e^{2}=0 \\
L_{e}(e x-e x e)=e(e x-e x e)=e(e x)-e(e x e)=e^{2} x-e^{2} x e=e x-e x e
\end{array}\right. \\
& \left\{\begin{array}{l}
R_{e}(x e-e x e)=x e^{2}-e x e^{2}=x e-e x e \\
L_{e}(x e-e x e)=e x e-e^{2} x e=0
\end{array}\right. \\
& \left\{\begin{array}{l}
R_{e}(x-e x-x e+e x e)=x e-e x e-x e^{2}+e x e^{2}=0 \\
L_{e}(x-e x-x e+e x e)=e x-e x-e x e+e x e=0
\end{array}\right.
\end{aligned}
$$

Mostrando que exe $\in A_{11},($ ex - exe $) \in A_{10},(x e-e x e) \in A_{01},(x-e x-x e+e x e) \in A_{00}$.

Antes de mostrarmos algumas propriedades da decomposição de Peirce para álgebras alternativas, precisaremos de uma notação auxiliar. Dados três elementos $x, y$ e $z$ de uma álgebra $A$, o associador dos elementos $x, y$ e $z$, denotado por $(x, y, z)$, é definido por $(x, y, z):=(x y) z-x(y z)$. Segue da bilinearidade do produto em uma álgebra que $k(x, y, z)=(k x, y, z)=(x, k y, z)=(x, y, k z)$ para qualquer $k \in K$. Além disso, $(x+u, y, z)=(x y) z+(u y) z-x(y z)-u(y z)=(x, y, z)+(u, y, z)$, analogamente, $(x, y+u, z)=(x, y, z)+(x, u, z)$ e $(x, y, z+u)=(x, y, z)+(x, y, u)$ para quaisquer $x, y, z, u \in A$. Se os elementos $x, y$ e $z$ pertencem a uma álgebra alternativa valem ainda as igualdades:

(1) $(x, x, y)=x^{2} y-x(x y)=0$;

(2) $(x, y, y)=(x y) y-x y^{2}=0$;

(3) $(y, x, z)=-(x, y, z)$;

(4) $(x, z, y)=-(x, y, z)$.

As igualdades (1) e (2) seguem diretamente da definição de álgebra alternativa. A igualdade (3) é decorrente das igualdades $(x+y, x+y, z)=(x, x, z)+(x, y, z)+(y, x, z)+(y, y, z)=$ $(x, y, z)+(y, x, z)=0$ e a igualdade (4) segue do mesmo modo se considerarmos o associador $(x, y+z, y+z)$.

Denotaremos por $x_{i j}$ um elemento de $A_{i j}$ onde $i, j=0,1$. Observe que $\left(x_{i j} y_{j i}\right) e=\left(x_{i j}, y_{j i}, e\right)+$ $x_{i j}\left(y_{j i} e\right)=-\left(x_{i j}, e, y_{j i}\right)+x_{i j}\left(y_{j i} e\right)=-j x_{i j} y_{j i}+j x_{i j} y_{j i}+i x_{i j} y_{j i}=i x_{i j} y_{j i}$. Analogamente, $e\left(x_{i j} y_{j i}\right)=i x_{i j} y_{j i}$. Isto mostra que $A_{i j} A_{j i} \subseteq A_{i i}$, isto é, $A_{11}$ e $A_{00}$ são subálgebras de $A$. Além disso, $x_{11} y_{00}=$ $\left(e x_{11} e\right) y_{00}=e\left(x_{11}\left(e y_{00}\right)\right)=0$, desse mesmo modo $y_{00} x_{11}=0$ o que mostra que $A_{11}$ e $A_{00}$ são subálgebras ortogonais de $A$. Aplicando o mesmo raciocínio podemos concluir que $A_{i i} A_{i j} \subseteq A_{i j}$ e $A_{i j} A_{j j} \subseteq A_{i j}$. Se $i \neq j$, então $x_{i j}^{2}=0$. Com efeito, $0=\left(x_{10}, e, x_{10}\right)=\left(x_{10} e\right) x_{10}-x_{10}\left(e x_{10}\right)=-x_{10}^{2}$, enquanto $0=\left(x_{01}, e, x_{01}\right)=\left(x_{01} e\right) x_{01}-x_{01}\left(e x_{01}\right)=x_{01}^{2}$.

Na próxima tabela, ilustramos como os subespaços da decomposição de Peirce para álgebras alternativas interagem em relação à inclusão. 


\begin{tabular}{||c|c|c|c|c||}
\hline$\subseteq$ & $A_{11}$ & $A_{10}$ & $A_{01}$ & $A_{00}$ \\
\hline$A_{11}$ & $A_{11}$ & $A_{10}$ & 0 & 0 \\
\hline$A_{10}$ & 0 & $A_{01}$ & $A_{11}$ & $A_{10}$ \\
\hline$A_{01}$ & $A_{01}$ & $A_{00}$ & $A_{10}$ & 0 \\
\hline$A_{00}$ & 0 & 0 & $A_{01}$ & $A_{00}$ \\
\hline
\end{tabular}

\subsection{Homomorfismos de Álgebras}

Intuitivamente um homomorfismo de álgebras é uma função entre duas álgebras que preserva as suas operações, ou seja, preserva a linearidade de espaço vetorial e a bilinearidade da multiplicação. A definição a seguir formaliza essa intuição.

Definição 1.12. Dadas $(A, \cdot)$ e $(B, *)$ duas $K$-álgebras, uma função $H: A \rightarrow B$ é chamada homomorfismo de álgebras se satisfizer as seguintes propriedades para quaisquer $u, v \in A$ e $k \in K$ :

1. $H$ é linear, isto é, $H(k u+v)=k H(u)+H(v)$;

2. $H$ preserva a multiplicação, ou seja, $H(u \cdot v)=H(u) * H(v)$.

Quando $H$ for injetivo, sobrejetivo ou bijetivo dizemos tratar-se respectivamente de um monomorfismo de álgebras, epimorfismo de álgebras ou isomorfismo de álgebras.

Dadas $(A, \cdot)$ e $(B, *)$ duas álgebras e $H$ um homomorfismo, se $A$ tem alguma das propriedades da Definição 1.7 então a álgebra $H(A)$ também a terá.

Exemplo 1.6. O homomorfismo trivial é a função $\theta: A \rightarrow B$ que satisfaz $\theta(v)=0$, para todo $v \in A$ e é um exemplo de homomorfismo que não é sobrejetivo ou injetivo a menos que $B$ ou $A$ possuam apenas o elemento 0 respectivamente.

Exemplo 1.7. Considerando o conjunto $\mathbb{R}$ como uma álgebra sobre si mesmo e fixando $\lambda \in \mathbb{R}$ temos um homomorfismo $\hat{\lambda}: \mathbb{R} \rightarrow \mathbb{R}$ definido por $\hat{\lambda}(v)=\lambda v$. Para $\lambda \neq 0$ este homomorfismo é um isomorfismo.

\subsection{Adjunção de elemento identidade}

Na Definição 1.7 vimos que nem toda álgebra possui identidade. O processo a seguir permite dotar de elemento identidade uma álgebra arbitrária.

Dada uma álgebra arbitrária $A$ sobre um corpo $K$, consideramos $K$ como uma $K$-álgebra sobre si mesmo e, assim, o elemento identidade $1_{K}$ de $K$ gera esta álgebra. Podemos então escrever: $K=K \cdot 1_{K}$. Consideramos agora a soma $A^{\#}=A \oplus K \cdot 1_{K}$ ou, simplesmente $A^{\#}=A \oplus K$, de duas $K$-álgebras e definimos suas operações da seguinte forma: Para quaisquer $\left(a_{1}, k_{1}\right),\left(a_{2}, k_{2}\right) \in A^{\#}$,

(1) - Soma de vetores em $A^{\#}:\left(a_{1}, k_{1}\right)+\left(a_{2}, k_{2}\right)=\left(a_{1}+a_{2}, k_{1}+k_{2}\right)$;

(2) - Produto por um escalar $\alpha \in K: \alpha\left(a_{1}, k_{1}\right)=\left(\alpha a_{1}, \alpha k_{1}\right)$; 
(3) - Multiplicação em $A^{\#}:\left(a_{1}, k_{1}\right)\left(a_{2}, k_{2}\right)=\left(a_{1} a_{2}+k_{2} a_{1}+k_{1} a_{2}, k_{1} k_{2}\right)$.

Observe que assim $A$ se torna uma subálgebra de $A^{\#}$ e esta última não ganha propriedades adicionais além de um elemento identidade, a saber, $1_{A^{\#}}=\left(0,1_{K}\right)$ e ter sua dimensão acrescida de um. Outras propriedades da adjunção de um elemento identidade e consequências fogem ao escopo deste trabalho, entretanto um tratado mais aprofundado pode ser consultado em [26]. Finalizamos esta subseção com um exemplo desta construção.

Exemplo 1.8. Considere a $\mathbb{R}$-álgebra gerada por $\{x\}$, denotada por $X$ com multiplicação definida pela relação $x^{2}=0$. Considere o $\mathbb{R}$-espaço vetorial $X \oplus \mathbb{R}$ com as operações definidas como nos itens (1),(2) e multiplicação definida por:

$$
\left(a_{1} x, r_{1}\right)\left(a_{2} x, r_{2}\right)=\left(r_{2} a_{1} x+r_{1} a_{2} x, r_{1} r_{2}\right)
$$

Desse modo, o elemento $(0,1)$ é tal que $(a x, r)(0,1)=(a x, r)$ e $(0,1)(a x, r)=(a x, r)$, isto é, este é o elemento identidade da multiplicação.

\subsection{O processo de duplicação de Cayley-Dickson}

A partir de uma $K$-álgebra unitária $A$ com uma involução $x \mapsto \bar{x}$ satisfazendo $x+\bar{x}, x \bar{x} \in K$ para todo $x \in A$ e fixando um elemento $a \in K$ tal que $a \neq 0$, podemos construir uma nova álgebra com involução que contenha A como uma subálgebra da seguinte forma:

(1) O espaço vetorial sobre o qual $(A, a)$ está definida é o espaço $A \oplus A$;

(2) A adição e multiplicação por escalar são definidas por: $\left(a_{1}, a_{3}\right)+\left(a_{2}, a_{4}\right)=\left(a_{1}+a_{2}, a_{3}+a_{4}\right)$ enquanto $k\left(a_{1}, a_{3}\right)=\left(k a_{1}, k a_{3}\right)$ para todo $k \in K$ e $a_{1}, a_{2}, a_{3}, a_{4} \in A$;

(3) A multiplicação é dada por: $\left(a_{1}, a_{3}\right)\left(a_{2}, a_{4}\right)=\left(a_{1} a_{2}+a a_{4} \overline{a_{3}}, \overline{a_{1}} a_{4}+a_{2} a_{3}\right)$.

Esta nova álgebra também possui uma involução, a saber, $\overline{\left(a_{1}, a_{2}\right)}=\left(\overline{a_{1}},-a_{2}\right)$.Vamos ilustrar este processo com um exemplo.

Exemplo 1.9. Considere o conjunto dos números reais como uma $\mathbb{R}$-álgebra munida das operações usuais e com a involução dada por $x \mapsto x$. Fixando $a=-1$, vamos construir a álgebra $(\mathbb{R},-1)$. O espaço vetorial sobre o qual definiremos as operações será $\mathbb{R} \oplus \mathbb{R}$ com as operações de adição de vetores e multiplicação por escalar usuais. A multiplicação é dada por $\left(x_{1}, y_{1}\right)\left(x_{2}, y_{2}\right)=\left(x_{1} x_{2}-y_{2} y_{1}, x_{1} y_{2}+x_{2} y_{1}\right)$ uma vez que a involução escolhida satisfaz $\bar{x}=x$. Para o que falta, vamos identificar a álgebra obtida com a $\mathbb{R}$-álgebra dos números complexos $\mathbb{C}$, isto é, provaremos que estas álgebras são isomorfas. Considere o morfismo $H: \mathbb{C} \rightarrow(\mathbb{R},-1)$ dado por $H(x+i y)=(x, y)$. Pela unicidade da expressão de um elemento $x \in \mathbb{C}$ em relação a base $\{1, i\}$ concluímos que $H$ está bem definido. É de verificação imediata que $H$ é linear e injetiva. Dados $u=x_{1}+i y_{1}$ e $v=x_{2}+i y_{2}$, temos que

$$
H(u) H(v)=H\left(x_{1}+i y_{1}\right) H\left(x_{2}+i y_{2}\right)=\left(x_{1}, y_{1}\right)\left(x_{2}, y_{2}\right)=\left(x_{1} x_{2}-y_{2} y_{1}, x_{1} y_{2}+x_{2} y_{1}\right)=H(u v) .
$$

Então $H$ é um homomorfismo injetivo entre $\mathbb{R}$-álgebras de dimensão finita, portanto é também sobrejetivo e consequentemente determina um isomorfismo entre $\mathbb{C}$ e $(\mathbb{R},-1)$. 


\section{Capítulo 2}

\section{O Comprimento de uma Álgebra}

Neste capítulo vamos denotar uma álgebra de dimensão finita sobre um corpo $\mathrm{K}$ (ou simplesmente uma K-álgebra) por $A$. Se $A$ for unitária, indicaremos o elemento identidade de $A$ por $1_{A}$. Além disso, dados dois conjuntos $A$ e $B$ de uma álgebra, o conjunto $\{a b: a \in A, b \in B\}$ será representado por $A \cdot B$.

Definição 2.1. Seja $S$ um subconjunto finito de uma $K$-álgebra $A$, uma palavra em $S$ é um produto finito de elementos de $S$, o comprimento de uma palavra é o número de fatores neste produto. Convencionamos ainda $1_{A}$ como uma palavra de comprimento zero.

Definição 2.2. Dado um subconjunto $S$ de uma $K$-álgebra $A$, o conjunto de todas as palavras em $S$ cujo comprimento não excede o valor $i$ será denotado por $S_{i}$; se a álgebra possuir elemento identidade convencionamos que $S_{0}=\left\{1_{A}\right\}$ ainda que $1_{A} \notin S$, caso contrário $S_{0}=\varnothing$. Vamos denotar o espaço vetorial gerado pelo conjunto $S_{i}$, ou seja, $\operatorname{span}\left\{S_{i}\right\}=\left\langle S_{i}\right\rangle$ por $\mathcal{L}_{i}(S)$, e por $\mathcal{L}(S)$ a união $\bigcup_{i \geq 0} \mathcal{L}_{i}(S)$

Decorre da Definição 2.2 que $\mathcal{L}_{0}(S)=\left\langle 1_{A}\right\rangle=K \cdot 1_{A}$ se a $K$-álgebra $A$ possuir identidade $1_{A}$ e $\mathcal{L}_{0}(S)=\langle\varnothing\rangle=\{0\}$ caso contrário.

Definição 2.3. Um subconjunto finito $S$ de uma $K$-álgebra $A$ será chamado de conjunto gerador quando $\mathcal{L}(S)=A$.

Uma álgebra de dimensão finita sempre possui um conjunto gerador, basta tomar uma base para o espaço vetorial sobre o qual está definida.

Exemplo 2.1. Sejam $A$ uma $K$-álgebra unitária e $S$ um subconjunto finito. Dada uma palavra $w$ em $S$, temos que $l(\alpha w)=l(w)$ para todo $\alpha \in K$.

Exemplo 2.2. Considerando $\mathbb{C}$ como álgebra sobre o corpo dos números reais munido do produto usual, o subconjunto $S=\{i\}$ satisfaz $S_{0}=\{1\}$ e $S_{1}=\{1, i\}$, o que implica $\mathcal{L}(S)=\mathbb{C}$ e $S$ é um conjunto gerador de $\mathbb{C}$.

Decorre da Definição 2.2 que $\mathcal{L}_{i}(S) \subseteq \mathcal{L}_{i+1}(S)$ para qualquer subconjunto $S$ de uma $K$-álgebra $A$. Em particular, pela finitude da dimensão de $A$ a cadeia de $K$-espaços vetoriais dada por $\mathcal{L}_{0}(S) \subseteq \mathcal{L}_{1}(S) \subseteq \ldots \subseteq \mathcal{L}_{n}(S) \subseteq \ldots$ estabiliza, isto é, existe um índice $k$ tal que $\mathcal{L}_{k}(S)=\mathcal{L}_{p}(S)$ para todo $p \geq k$. A existência de tal índice $k$ possibilita a seguinte definição. 
Definição 2.4. Dado um conjunto gerador $S$ de uma $K$-álgebra $A$, o comprimento do conjunto $S$, denotado por $l(S)$, é o menor inteiro não negativo $n$ tal que é válida a relação $\mathcal{L}_{n}(S)=A$, considerados como espaços vetoriais.

Exemplo 2.3. O comprimento do conjunto $S$ no Exemplo 2.2 é 1 pois $\mathcal{L}_{1}(S)=\mathbb{C}$ e $\mathcal{L}_{0}(S) \neq \mathbb{C}$.

Definição 2.5. Seja $S$ um conjunto gerador de uma $K$-álgebra $A$ de dimensão finita $m$. Dizemos que a cadeia de $S$ é a sequência de inteiros não negativos $a_{0} \rightarrow a_{1} \rightarrow \ldots \rightarrow a_{n}$, onde $a_{i}=\operatorname{dim} \mathcal{L}_{i}(S)$, $0 \leq i \leq n=l(S)$.

Retornando ao Exemplo 2.2, a cadeia do conjunto gerador $S$ é dada por $1 \rightarrow 2$ uma vez que $\operatorname{dim} \mathcal{L}_{0}(S)=1$ e $\operatorname{dim} \mathcal{L}_{1}(S)=2$.

Definição 2.6. O comprimento de uma álgebra $A$ é o maior inteiro, dentre todos os comprimentos de conjuntos geradores de $A$, isto é, $l(A)=\max \{l(S): \mathcal{L}(S)=A\}$.

Exemplo 2.4. Considerando a álgebra do Exemplo 2.2, vamos determinar seu comprimento. Já determinamos a existência de um conjunto $S$ tal que $l(S)=1$, suponha que exista um subconjunto $R$ satisfazendo $l(R)>1$. Como $R_{0}=\{1\}$, para que $\operatorname{dim} \mathcal{L}_{1}(R) \leq 1$ devemos ter que os vetores de $R_{1}$ são linearmente dependentes, neste caso, múltiplos de 1 o que implica $\operatorname{dim} \mathcal{L}_{n}(R)<2$ para todo $n>1$ portanto $R$ não é gerador, concluímos assim que $l(A)=1$.

Definição 2.7. Seja $R$ um subconjunto finito de uma $K$-álgebra $A$. Dizemos que $R$ é refinado se é linearmente independente e se está livre de elementos pertencentes a $K \cdot 1_{A}$.

Para obter o refinamento de um subconjunto finito $R$, inicialmente tomamos $R^{1}=R \backslash(R \cap$ $\left.K .1_{A}\right)$. Se $R^{1}$ for um conjunto linearmente independente, então $R^{1}$ é um refinamento de $R$. Se existir um vetor $v_{1} \in R^{1}$ que é combinação linear dos demais, fazemos $R^{2}=R^{1} \backslash\left\{v_{1}\right\}$. Repetimos o mesmo procedimento com $R^{2}$ e prosseguimos iterativamente até obter um conjunto linearmente independente $R^{n} \subseteq R$, que será um conjunto refinado.

Como este processo pode depender da escolha de elementos a serem retirados, um refinamento $R^{\prime}$ de um conjunto $R$ nem sempre é único mas todos são minimais em relação à cardinalidade, possuem a mesma cadeia que o conjunto original e além disso, vale a relação $a_{1}=\left|R^{\prime}\right|+a_{0}$.

Exemplo 2.5. Considerando $A=\mathbb{C}$ como álgebra sobre o corpo dos números reais e $S=\{2,1+i, i\}$, vamos determinar um refinamento de $S$. Na primeira etapa retiramos o elemento $\{2\}$ do conjunto $S$ pois a álgebra é unitária, e obtemos $S^{1}=\{1+i, i\}$. Como os vetores $1+i$ e $i$ são linearmente independentes o processo termina na etapa 1 e podemos concluir que $S^{\prime}=\{1+i, i\}$ é um refinamento de $S$.

\subsection{Propriedades do comprimento}

Proposição 2.1. Sejam $m<n$ inteiros positivos e $S$ um subconjunto qualquer. São equivalentes as seguintes afirmações:

1. $\mathcal{L}_{m}(S)=\mathcal{L}_{n}(S)$; 
2. $\operatorname{dim} \mathcal{L}_{m}(S)=\operatorname{dim} \mathcal{L}_{n}(S)$.

Demonstração. Se $\mathcal{L}_{m}(S)=\mathcal{L}_{n}(S)$, então claramente $\operatorname{dim} \mathcal{L}_{m}(S)=\operatorname{dim} \mathcal{L}_{n}(S)$. Reciprocamente, se $\operatorname{dim} \mathcal{L}_{m}(S)=\operatorname{dim} \mathcal{L}_{n}(S)$, então como $\mathcal{L}_{m}(S)$ e $\mathcal{L}_{n}(S)$ são subespaços vetoriais e $\mathcal{L}_{m}(S) \subseteq \mathcal{L}_{n}(S)$, podemos concluir que $\mathcal{L}_{m}(S)=\mathcal{L}_{n}(S)$.

Corolário 2.1. Se $\operatorname{dim} \mathcal{L}_{0}(S)=\operatorname{dim} \mathcal{L}_{1}(S)=1$ então $\mathcal{L}(S)=K \cdot 1_{A}$.

Demonstração. Se $\operatorname{dim} \mathcal{L}_{0}(S)=\operatorname{dim} \mathcal{L}_{1}(S)=1$, então $K \cdot 1_{A}=\mathcal{L}_{0}(S)=\mathcal{L}_{1}(S)$. Desse modo, $S$ consiste apenas de elementos da forma $\alpha 1_{A} \operatorname{com} \alpha \in K$. Assim, $\mathcal{L}_{n}(S)=K \cdot 1_{A}$ para todo $n>0$.

Proposição 2.2. [7] Sejam $R$ e $S$ subconjuntos finitos de uma $K$-álgebra $A$ tais que $\mathcal{L}_{1}(R) \subseteq \mathcal{L}_{1}(S)$. Então $\mathcal{L}_{k}(R) \subseteq \mathcal{L}_{k}(S)$ para todo inteiro positivo $k$.

Demonstração. A prova será feita por indução no índice $k$. O caso base $k=1$ é válido por hipótese. Suponha agora que $\mathcal{L}_{k}(R) \subseteq \mathcal{L}_{k}(S)$ para $1 \leq k \leq n-1$ e vejamos que $\mathcal{L}_{n}(R) \subseteq \mathcal{L}_{n}(S)$. Pelas nossas definições sabemos que: $\mathcal{L}_{n}(R)=\operatorname{span}\left(\bigcup_{i=1}^{n-1} \mathcal{L}_{i}(R) \cdot \mathcal{L}_{n-i}(R)\right)$. Assim, como $\mathcal{L}_{i}(R) \subseteq$ $\mathcal{L}_{i}(S)$ e $\mathcal{L}_{n-i}(R) \subseteq \mathcal{L}_{n-i}(S)$ para $1 \leq i \leq n-1$, pela hipótese de indução concluímos que $\mathcal{L}_{n}(R) \subseteq$ $\operatorname{span}\left(\bigcup_{i=1}^{n-1} \mathcal{L}_{i}(S) \cdot \mathcal{L}_{n-i}(S)\right)=\mathcal{L}_{n}(S)$

Corolário 2.2. [7] Sejam $R$ e $S$ subconjuntos geradores de uma $K$-álgebra $A$ tais que $\mathcal{L}_{1}(R) \subseteq \mathcal{L}_{1}(S)$, então $l(R) \geq l(S)$.

Demonstração. Aplicando a Proposição 2.2 sabemos que $\mathcal{L}_{l(S)-1}(R) \subseteq \mathcal{L}_{l(S)-1}(S) \neq A$. Assim, como ambos os conjuntos são geradores e $\mathcal{L}_{l(S)}(R) \subseteq \mathcal{L}_{l(S)}(S)=A$, temos que $l(R) \geq l(S)$.

Corolário 2.3. [7] Se $R$ e $S$ são subconjuntos finitos de uma $K$-álgebra $A$, tais que $\mathcal{L}_{1}(R)=\mathcal{L}_{1}(S)$, então $\mathcal{L}_{k}(R)=\mathcal{L}_{k}(S)$ para todo inteiro positivo $k$.

Aplicando duas vezes a Proposição 2.2, temos que o seguinte resultado é válido.

Corolário 2.4. [7] Sejam $R \subset A$ um subconjunto gerador e $S \subset A$ um subconjunto finito de uma $K$-álgebra $A$ tais que $\mathcal{L}_{1}(R)=\mathcal{L}_{1}(S)$ então $S$ também é gerador de $A$ e $l(R)=l(S)$.

Corolário 2.5. [7] Se $S$ é um conjunto gerador de uma $K$-álgebra $A$ e $S^{\prime}$ é um refinamento de $S$, então $l(S)=l\left(S^{\prime}\right)$.

Demonstração. É uma consequência imediata do Corolário 2.4, pois $\mathcal{L}_{1}(S)=\mathcal{L}_{1}\left(S^{\prime}\right)$.

Proposição 2.3. [7] Sejam $A$ uma $K$-álgebra não unitária e $A^{\#}=A \oplus K \cdot 1$ a álgebra unitária obtida pelo processo de adjunção de elemento identidade. Se $S=\left\{\left(a_{1}, f_{1}\right), \ldots,\left(a_{n}, f_{n}\right)\right\}$ é um subconjunto gerador de $A^{\#}$, então o conjunto $R=\left\{\left(a_{1}, 0\right), \ldots,\left(a_{n}, 0\right)\right\}$ também é gerador e a igualdade $l(S)=$ $l(R)$ é válida.

Demonstração. Pelo Corolário 2.4, basta mostrar que $\mathcal{L}_{1}(S)=\mathcal{L}_{1}(R)$. Seja $x \in S_{1}$. Se $x=(0,1)$, então $x \in R_{1}$, por definição; caso contrário, existem $a_{j} \in A$ e $f_{j} \in K$, para algum $j \in\{1, \ldots, n\}$ tais que $s=\left(a_{j}, f_{j}\right)=\left(a_{j}, 0\right)+f_{j}(0,1) \in \mathcal{L}_{1}(R)$. Isso mostra que $S \subset \mathcal{L}_{1}(R)$ e então $\mathcal{L}_{1}(S) \subseteq \mathcal{L}_{1}(R)$. Reciprocamente, dado $r \in R_{1}$, se $r=(0,1)$, então já sabemos que $r \in S_{1}$; do contrário, existe $a_{j} \in A$, para algum $1 \leq j \leq n$, tal que $r=\left(a_{j}, 0\right)=\left(a_{j}, f_{j}\right)-f_{j}(0,1) \in \mathcal{L}_{1}(S)$, argumentamos de modo similar ao caso anterior e concluímos que $\mathcal{L}_{1}(R) \subseteq \mathcal{L}_{1}(S)$. 
Proposição 2.4. [7] Se $A$ é uma álgebra não unitária e $A^{\#}$ é a álgebra unitária obtida pelo processo de adjunção de elemento identidade, então $l(A)=l\left(A^{\#}\right)$.

Demonstração. A desigualdade $l(A) \leq l\left(A^{\#}\right)$ é uma consequência imediata da Proposição 2.3. Para provar a outra desigualdade, seja $S_{1}=\left\{b_{1}, \ldots, b_{k}\right\}$ um conjunto gerador tal que $l\left(S_{1}\right)=l\left(A^{\#}\right)$. Por definição $b_{i}=\left(a_{i}, f_{i}\right)$ onde $a_{i} \in A$ e $f_{i} \in K$. Consideramos agora o conjunto $P=\left\{\left(a_{1}, 0\right), \ldots,\left(a_{k}, 0\right)\right\}$, observe que, em virtude da Proposição 2.3, a igualdade $l(P)=l\left(S_{1}\right)$ é verdadeira. Denote por $S=\left\{a_{1}, \ldots, a_{k}\right\}$. Observe agora que

$$
\mathcal{L}_{m}(S)=\left\langle S_{m}\right\rangle \simeq\left\langle\left\{(v, 0): v \in S_{m}\right\}\right\rangle=\left\langle P_{m} \backslash P_{0}\right\rangle
$$

e, pela definição de $P_{0}$, temos que $\mathcal{L}_{m}(P)=\left\langle P_{m} \backslash P_{0}\right\rangle \oplus\left\langle P_{0}\right\rangle$. Consequentemente $\operatorname{dim} \mathcal{L}_{m}(P)=$ $\operatorname{dim} \mathcal{L}_{m}(S)+1$ para qualquer $m \geq 1$. Deste modo, para $m=l\left(S_{1}\right)$ temos que $\operatorname{dim} \mathcal{L}_{m}(S)=\operatorname{dim} \mathcal{L}_{m}(P)-$ $1=\operatorname{dim}\left(A^{\#}\right)-1=\operatorname{dim} A, \operatorname{assim} l\left(A^{\#}\right)=l(P)=l(S) \leq l(A)$, concluindo a demonstração.

Lema 2.1. [7] Sejam $S$ um subconjunto finito qualquer de uma álgebra $A$ e $n$ um inteiro positivo. $\operatorname{Se} \operatorname{dim} \mathcal{L}_{n}(S)=\operatorname{dim} \mathcal{L}_{n+1}(S)=\cdots=\operatorname{dim} \mathcal{L}_{2 n}(S)$, então $\operatorname{dim} \mathcal{L}_{n}(S)=\operatorname{dim} \mathcal{L}_{n+k}(S)$ para qualquer inteiro positivo $t$.

Demonstração. A prova será por indução no acréscimo $t$ do índice $n$. O caso base $t \leq n$ é válido pelas hipóteses da proposição. Suponhamos que o resultado seja válido para quaisquer $t \leq n+$ $k, k \geq 0$ e vamos verificá-lo para $t=n+k+1$.

Se $s$ é uma palavra de comprimento $n+k+1$ então $s$ pode ser representada como um produto de palavras de comprimentos menores que $l(s)$ e maiores que 1 , isto é, $s=\left(s_{1}\right)\left(s_{2}\right)$, onde $1 \leq$ $l\left(s_{1}\right) \leq l(s)$ e $1 \leq l\left(s_{2}\right) \leq l(s)$. Afirmamos que $s_{1}$ e $s_{2}$ são elementos de $\mathcal{L}_{n}(S)$. Com efeito, se o comprimento de uma palavra é menor ou igual a $n$, tal palavra já é um elemento de $\mathcal{L}_{n}(S)$. Pela definição de $\mathcal{L}_{n}(S)$, se o comprimento de uma palavra é maior do que $n$, mas estritamente do que $n+k+1$, então tal palavra pertence a $\mathcal{L}_{m}(S)$ para algum $n<m<n+k+1$, que pela hipótese de indução coincide com $\mathcal{L}_{n}(S)$. Desse modo, $s \in \mathcal{L}_{n}(S) \cdot \mathcal{L}_{n}(S) \subseteq \mathcal{L}_{2 n}(S)=\mathcal{L}_{n}(S)$. Portanto $\mathcal{L}_{n+k+1}(S) \subseteq \mathcal{L}_{n}(S)$. Assim, como a inclusão reversa é sempre válida, segue que $\mathcal{L}_{n+k+1}(S)=\mathcal{L}_{n}(S)$, o que conclui a demonstração.

Para mostrar a necessidade das igualdades $\operatorname{dim} \mathcal{L}_{n}(S)=\operatorname{dim} \mathcal{L}_{n+1}(S)=\cdots=\operatorname{dim} \mathcal{L}_{2 n}(S)$ utilizamos o exemplo a seguir.

Exemplo 2.6. Considere a álgebra $A$ gerada por $\left\{1_{A}, e_{1}, e_{2}, e_{3}\right\}$ com a tábua de multiplicação:

\begin{tabular}{||c|c|c|c|c||}
\hline$*$ & 1 & $e_{1}$ & $e_{2}$ & $e_{3}$ \\
\hline 1 & 1 & $e_{1}$ & $e_{2}$ & $e_{3}$ \\
\hline$e_{1}$ & $e_{1}$ & $e_{2}$ & 0 & 0 \\
\hline$e_{2}$ & $e_{2}$ & 0 & $e_{3}$ & 0 \\
\hline$e_{3}$ & $e_{3}$ & 0 & 0 & 0 \\
\hline
\end{tabular}

Neste caso temos que $S=\left\{e_{1}\right\}$ é um conjunto gerador e que $\mathcal{L}_{1}(S)=\left\langle 1, e_{1}\right\rangle, \mathcal{L}_{2}(S)=\left\langle 1, e_{1}, e_{2}\right\rangle, \mathcal{L}_{3}(S)=$ $\left\langle 1, e_{1}, e_{2}\right\rangle, \mathcal{L}_{4}(S)=\left\langle 1, e_{1}, e_{2}, e_{3}\right\rangle=A$. Portanto $\operatorname{dim} \mathcal{L}_{4}(S)=4>\operatorname{dim} \mathcal{L}_{3}(S)=\mathcal{L}_{2}(S)$. 
Para valores maiores de $n$ podemos estender o caso do exemplo anterior como será feito no Exemplo 2.9, comprovando que é impossível reduzir as hipóteses do Lema 2.1.

Em vista de que o Lema 2.1 será usado exaustivamente no Capítulo 3, vamos ilustrar a sua importância com algumas aplicações.

Aplicação 1. Uma álgebra de dimensão 3 não pode ter comprimento 5.

Demonstração. Com efeito, seja $A$ uma álgebra tal que $\operatorname{dim} A=3$ e $l(A)=5$. Deste modo, existe um conjunto gerador $S$ de $A$ tal que 5 é o maior inteiro positivo que satisfaz a relação $\mathcal{L}_{5}(S)=A$. Se $S=\varnothing$ ou $S=\{0\}$ então $S$ não é um conjunto gerador de $A$. Desse modo, $S$ possui pelo menos um elemento não nulo. Neste caso, $\operatorname{dim} \mathcal{L}_{1}(S) \geq 1$. Se $\operatorname{dim} \mathcal{L}_{1}(S)=1$, então pelo Lema $2.1, \operatorname{dim} \mathcal{L}_{2}(S) \geq 2$ e $\operatorname{dim} \mathcal{L}_{4}(S) \geq 3$, o que é uma contradição pois neste caso, $\mathcal{L}_{4}(S)=A$. Se $\operatorname{dim} \mathcal{L}_{1}(S)=2$, então pelo Lema 2.1, $\operatorname{dim} \mathcal{L}_{2}(S) \geq 3$, o que implicaria em $\mathcal{L}_{2}(S)=A$, o que novamente é uma contradição. Pelos mesmos argumentos, sabemos que $\operatorname{dim} \mathcal{L}_{1}(S)<3$. Portanto, não existe uma álgebra de dimensão 3 e comprimento 5 .

Aplicação 2. Sejam $A$ uma $K$-álgebra unitária de dimensão $n \geq 1$ e $S$ um conjunto gerador refinado de $A$. Então $l(S) \leq 2^{n-2}$. Em particular, $l(A) \leq 2^{n-2}$.

Demonstração. Quando $n=1$, vale que $A=K \cdot 1_{A}$ e então $\operatorname{dim} \mathcal{L}_{0}(S)=1$ para qualquer subconjunto S. Segue dessa observação que $l(A)=0 \leq \frac{1}{2}$.

Suponha $n>1$. Se existisse um conjunto gerador $S$ satisfazendo $l(S)>2^{n-2}$, então

$$
n>a_{2^{n-2}}=a_{1}+\sum_{j=1}^{n-2}\left(a_{2^{j}}-a_{2^{j-1}}\right) \geq a_{1}+\sum_{j=1}^{n-2} 1=a_{1}+n-2,
$$

o que implicaria $2>\operatorname{dim} \mathcal{L}_{1}(S)$, um absurdo.

Uma consequência imediata da Aplicação 2 é que $l(A) \leq 2^{n-1}$ se $A$ não possuir elemento identidade e $l(A) \leq 2^{n-2}$ caso contrário.

Aplicação 3. Sejam $A$ uma $K$-álgebra unitária de dimensão $n>2$ que é de potências associativas, mas não é alternativa, e $S$ um conjunto gerador refinado de $A$. Então $l(S) \leq 2^{n-3}$. Em particular, $l(A) \leq 2^{n-3}$.

Demonstração. Se existir um subconjunto gerador $S$ de $A$ tal que $l(S)>2^{n-3}$, então

$$
n>a_{2^{n-3}}=a_{1}+\sum_{j=1}^{n-3}\left(a_{2^{j}}-a_{2^{j-1}}\right) \geq a_{1}+\sum_{j=1}^{n-3} 1=a_{1}+n-3
$$

isto é, $\operatorname{dim} \mathcal{L}_{1}(S)<3$. Portanto, $\operatorname{dim} \mathcal{L}_{1}(S)=2$, o que significa que $\mathcal{L}_{1}(S)=\langle 1, x\rangle$, para algum $x \in A$. Consequentemente, $S=\{x\}$ e como $A$ é uma álgebra de potências associativas, podemos concluir que $A$ é uma álgebra associativa, o que é uma contradição, pois $A$ não é uma álgebra alternativa.

Corolário 2.6. Toda álgebra de potências associativas, unitária, com dimensão $n>2$ e que possui um conjunto gerador de comprimento maior que $2^{n-3}$ é associativa. 
Novamente, aplicando o mesmo raciocínio, podemos concluir que se $\operatorname{dim} A>2$ e $A$ satisfaz as hipóteses da Aplicação 3, então $l(A) \leq 2^{n-2}$ se $A$ não possui elemento identidade e $l(A) \leq 2^{n-3}$ caso contrário.

Aplicação 4. Sejam $A$ uma $K$-álgebra unitária de dimensão $n>3$ que é alternativa, mas não é associativa, e $S$ um subconjunto gerador refinado de $A$. Então $l(S) \leq 2^{n-4}$. Em particular, $l(A) \leq 2^{n-4}$.

Demonstração. Se existir um subconjunto gerador $S$ de $A$ tal que $l(S)>2^{n-4}$, então

$$
n>a_{2^{n-4}}=a_{1}+\sum_{j=1}^{n-4}\left(a_{2^{j}}-a_{2^{j-4}}\right) \geq a_{1}+\sum_{j=1}^{n-4} 1=a_{1}+n-4
$$

isto é, $\operatorname{dim} \mathcal{L}_{1}(S)<4$. Portanto, $\operatorname{dim} \mathcal{L}_{1}(S) \in\{2,3\}$, o que significa que $\mathcal{L}_{1}(S)=\langle 1, x\rangle$ ou $\mathcal{L}_{1}(S)=$ $\langle 1, x, y\rangle$, para alguns $x, y \in A$. Consequentemente, $S=\{x, y\}$ e como $A$ é uma álgebra alternativa, pelo Teorema 5.1, a subálgebra gerada por dois elementos é associativa, podemos concluir que $A$ é uma álgebra associativa, o que é uma contradição.

Corolário 2.7. Toda álgebra unitária, alternativa, com dimensão $n>3$ e que possui um conjunto gerador de comprimento maior que $2^{n-4}$ é associativa.

Novamente, quando a álgebra estiver nas hipóteses da Aplicação 4 e $\operatorname{dim} A>3$, podemos concluir que $l(A) \leq 2^{n-3}$ se $A$ não possui elemento identidade e $l(A) \leq 2^{n-4}$ caso contrário.

\subsection{Palavras Irredutíveis}

Definição 2.8. Uma palavra $w$ de comprimento $n$ de um conjunto gerador $S \subset A$ é irredutível, se para todo inteiro $m, 0 \leq m<n$, valer que $w \notin \mathcal{L}_{m}(S)$.

Exemplo 2.7. A palavra $w=\left(e_{1} e_{1}\right)\left(e_{1} e_{1}\right)=e_{3}$ de comprimento 4 do conjunto gerador $S$ considerado no Exemplo 2.6 é uma palavra irredutível pois, $e_{3} \notin \mathcal{L}_{m}(S), 0 \leq m \leq 3$. Além disso, $w$ é o produto de outras duas palavras irredutíveis pois, $e_{2}=e_{1} e_{1} \notin \mathcal{L}_{n}(S)$ para $n=1$, o que não é uma casualidade como veremos no próximo resultado.

Lema 2.2. [7] Uma palavra irredutível de comprimento maior que um é o produto de duas palavras irredutíveis de comprimentos não nulos.

Demonstração. Já sabemos que uma palavra qualquer de comprimento maior que um e, assim, em particular, uma palavra irredutível pode ser escrita como um produto de duas palavras $s$ e $t$ de comprimentos $a>0$ e $b>0$, respectivamente.

Assuma que $s$ não seja uma palavra irredutível. Desse modo, existe um número inteiro $0 \leq a^{\prime}<a$ tal que $s \in \mathcal{L}_{a^{\prime}}(S)$. Assim, $w$ é uma palavra de comprimento $a+b$ tal que $w \in \mathcal{L}_{a^{\prime}+b}(S)$, o que contradiz a irredutibilidade da palava $w$. Portanto, s é uma palavra irredutível. Pelos mesmos argumentos, $t$ também é uma palavra irredutível e o lema está provado.

A análise da existência de palavras irredutíveis é uma ferramenta importante que usaremos inúmeras vezes no decorrer desta dissertação. Uma ilustração inicial de sua relevância pode ser comprovada no seguinte resultado. 
Exemplo 2.8. Se $A$ uma álgebra não unitária de dimensão 8 , então $A$ não possui subconjunto gerador $S$ tal que $\operatorname{dim} \mathcal{L}_{0}(S)=0, \operatorname{dim} \mathcal{L}_{1}(S)=n, \operatorname{dim} \mathcal{L}_{2}(S)=n+1, \operatorname{dim} \mathcal{L}_{3}(S)=n+1$ e $\operatorname{dim} \mathcal{L}_{4}(S)=8$, para qualquer $0<n<6$.

Com efeito, se tal conjunto gerador existisse, como $\operatorname{dim} \mathcal{L}_{3}(S)=n+1 \leq 6$ e $\operatorname{dim} \mathcal{L}_{4}(S)=8$, haveriam pelo menos duas palavras linearmente independentes em $\mathcal{L}_{4}(S)$ que pelo Lema 2.2 teriam que ser escritas como produto de duas palavras de comprimento 2 , pois como $\operatorname{dim} \mathcal{L}_{2}(S)=\operatorname{dim} \mathcal{L}_{4}(S)$ não existiriam palavras irredutíveis de comprimento 3. Entretanto, como $\operatorname{dim} \mathcal{L}_{2}(S)=\mathcal{L}_{1}(S)+1$, somente existiria uma palavra irredutível de comprimento 2 , o que tornaria impossível haverem duas palavras irredutíveis distintas de comprimento 4 .

\subsubsection{Sequências características e suas propriedades básicas}

Nesta seção vamos introduzir uma técnica primordial para considerações futuras. Salvo menção contrária, todas as álgebras consideradas nesta seção serão unitárias.

Definição 2.9. Sejam $A$ uma $K$-álgebra com elemento identidade $1_{A}$, de dimensão $n$ e $S$ um conjunto gerador. A sequência característica de $S$ em $A$ é uma sequência monótona não decrescente de números naturais $\left(m_{0}, \ldots, m_{p}\right)$ construída da seguinte forma:

(i) $m_{0}=0$;

(ii) Denotando $s_{1}=\operatorname{dim} \mathcal{L}_{1}(S)-1$, definimos $m_{1}=\cdots=m_{s_{1}}=1$;

(iii) Se os elementos $m_{1}, \ldots, m_{r}$ já estão construídos e os conjuntos $\mathcal{L}_{1}(S), \ldots, \mathcal{L}_{k}(S)$ já foram considerados, prosseguimos indutivamente considerando $s_{k}=\operatorname{dim} \mathcal{L}_{k}(S)-\operatorname{dim} \mathcal{L}_{k-1}(S)$ e adicionamos os termos $m_{r+1}=\ldots=m_{r+s_{k}}=s_{k}$ na sequência.

Em outras palavras, para construir a sequência característica, começamos em $m_{0}=0$ e para cada $k=1, \ldots, l(A)$ nós adicionamos $\operatorname{dim} \mathcal{L}_{k}(S)-\operatorname{dim} \mathcal{L}_{k-1}(S)$ elementos iguais a $k$.

Vamos retomar o Exemplo 2.2 e determinar a sequência característica do conjunto $S$ lá considerado. Começamos em $m_{0}=0$ e, após isso, calculamos $s_{1}=\operatorname{dim} \mathcal{L}_{1}(S)-1=1$, adicionando um único termo cujo valor é 1 à sequência. Portanto, a sequência característica de $S$ é dada por $(0,1)$.

Sempre é possível determinar a sequência característica de um conjunto gerador $S$ conhecendo sua cadeia. Considere, por exemplo, a cadeia $1 \rightarrow 3 \rightarrow 4 \rightarrow 5$. Por um lado, se a álgebra cuja cadeia está determinada é unitária, prosseguimos de acordo com a Definição 2.9: $m_{0}=0$, $s_{1}=a_{1}-1=2 \operatorname{assim} m_{1}=m_{2}=1, s_{2}=a_{2}-a_{1}=1$ então $m_{3}=2$ e por fim $s_{3}=a_{3}-a_{2}=1$ e $m_{4}=3$. Construímos assim a sequência característica $(0,1,1,2,3)$ de $S$. Por outro lado, se a álgebra em questão não for unitária, realizamos a adjunção de elemento identidade, o que incrementa em um o valor de cada elemento $a_{i}$ presente na cadeia de $S$ e prosseguimos como no exemplo anterior.

Lema 2.3. [7] Sejam $A$ uma álgebra tal que $\operatorname{dim} A=n>2$ e $S$ um conjunto gerador de $A$. As seguintes afirmações são verdadeiras.

i) O inteiro positivo $k$ aparece na sequência característica o mesmo número de vezes que a quantidade de palavras irredutíveis linearmente independentes de comprimento $k$; 
ii) Para qualquer termo $m_{h}$ da sequência característica de $S$ existe uma palavra irredutível em $\mathcal{L}(S)$ de comprimento $m_{h}$;

iii) Se existir uma palavra irredutível em $\mathcal{L}(S)$ de comprimento $k$, então $k$ está presente na sequência característica de $S$.

Demonstração. i) As palavras irredutíveis de comprimento no máximo $k$ formam uma base para $\mathcal{L}_{k}(S)$, e assim, a quantidade de palavras linearmente independentes de comprimento $k$ é dada por $\operatorname{dim} \mathcal{L}_{k}(S)-\operatorname{dim} \mathcal{L}_{k-1}(S)$. As afirmações ii) e iii) decorrem de i).

Lema 2.4. [7] Sejam $A$ uma álgebra tal que $\operatorname{dim} A=n>2$ e $S$ um conjunto gerador de $A$. Então a sequência característica de $S$ contém $n$ termos e $l(S)=m_{n-1}$.

Demonstração. Por definição, para cada $k=1, \ldots, l(S)$ na $k$-ésima etapa adicionamos $\left(\operatorname{dim} \mathcal{L}_{k}(S)-\right.$ $\left.\operatorname{dim} \mathcal{L}_{k-1}(S)\right)$ termos à sequência característica. Assim, o número total de termos é

$$
1+\left(\operatorname{dim} \mathcal{L}_{1}(S)-1\right)+\cdots+\left(\operatorname{dim} \mathcal{L}_{l(S)}(S)-\operatorname{dim} \mathcal{L}_{l(S)-1}(S)\right)=n,
$$

pois a soma é telescópica e $S$ é um conjunto gerador. Além disso, pela definição de comprimento de $S$, o valor $k$ maximal tal que $\operatorname{dim} \mathcal{L}_{k}(S)-\operatorname{dim} \mathcal{L}_{k-1}(S)>0$ é $l(S)$. Portanto, pela Definição 2.9, concluímos que $m_{n-1}=l(S)$.

Lema 2.5. [7] Sejam $A$ uma álgebra tal que $\operatorname{dim} A=n>2, S$ um conjunto gerador de $A$ e $\left(m_{0}, \ldots, m_{n-1}\right)$ a sequência característica de $S$. Então para cada $h$ satisfazendo $m_{h} \geq 2$ existem índices $0<t_{1} \leq t_{2}<h$ tais que $m_{h}=m_{t_{1}}+m_{t_{2}}$.

Demonstração. Pelo item i) do Lema 2.3, cada termo $m_{h}$ da sequência característica corresponde a uma palavra irredutível de comprimento $m_{h}$, que vamos denotar por $w_{m_{h}}$. Pelo Lema 2.2, cada palavra irredutível de comprimento $m_{h} \geq 2$ pode ser escrita como o produto de duas palavras não necessariamente distintas de comprimentos menores. Desse modo, $w_{m_{h}}=w_{k_{1}} w_{k_{2}}$, onde $w_{k_{1}}$ e $w_{k_{2}}$ são palavras irredutíveis tais que $l\left(w_{k_{1}}\right)=k_{1}<m_{h}$ e $l\left(w_{k_{2}}\right)=k_{2}<m_{h}$. Por um lado, se $k_{1} \leq k_{2}$, então pelo item iii) do Lema 2.3, existem índices $0<t_{1} \leq t_{2}<h$ tais que $m_{t_{1}}=k_{1}$ e $m_{h_{2}}=k_{2}$. Por outro lado, se $k_{1}>k_{2}$, então novamente pelo item iii) do Lema 2.3, existem índices $0<t_{1} \leq t_{2}<h$ tais que $m_{t_{1}}=k_{2}$ e $m_{t_{2}}=k_{1}$. Em ambos os casos, pela aditividade do comprimento de palavras, temos que $l\left(w_{m_{h}}\right)=l\left(w_{k_{1}}\right)+l\left(w_{k_{2}}\right)=k_{1}+k_{2}=m_{t_{1}}+m_{t_{2}}=m_{t_{2}}+m_{t_{1}}$, o que conclui a demonstração.

Lema 2.6. [7] Sejam $A$ uma $K$-álgebra $\operatorname{com} \operatorname{dim} A=n>2, S$ um conjunto gerador e $\left(m_{0}, m_{1}, \cdots, m_{n-1}\right)$ a sequência característica de $S$. Então para cada inteiro $k \geq 0$, vale que $\operatorname{dim} \mathcal{L}_{k}(S)=\max \left\{t: m_{t} \leq\right.$ $k\}+1$.

Demonstração. Vamos proceder por indução em $k$. Para $k=0$, a afirmação é válida trivialmente, pois $\operatorname{dim} \mathcal{L}_{0}(S)=1=m_{0}+1$. Assumimos que a afirmação seja verdadeira para $k=q$, isto é, $\operatorname{dim} \mathcal{L}_{q+1}(S)=\operatorname{dim} \mathcal{L}_{q}(S)=\max \left\{t: m_{t} \leq q\right\}+1$. Para $k=q+1$, é claro que $\operatorname{dim} \mathcal{L}_{q+1}(S)=\left(\operatorname{dim} \mathcal{L}_{q+1}(S)\right.$ $\left.-\operatorname{dim} \mathcal{L}_{q}(S)\right)+\operatorname{dim} \mathcal{L}_{q}(S)$. Agora, pela Definição 2.9, o somando $\left(\operatorname{dim} \mathcal{L}_{q+1}(S)-\operatorname{dim} \mathcal{L}_{q}(S)\right)$ é igual ao número $N_{0}$ de termos iguais a $(q+1)$ na sequência característica. Além disso, pela hipótese de indução, $N_{1}=\operatorname{dim} \mathcal{L}_{q}(S)$ é o incremento por 1 da última posição em que $m_{t} \leq q$. Pela Definição 2.9 a soma $N_{0}+N_{1}$ é igual ao incremento por 1 da última posição em que $m_{t} \leq q+1$, ou seja, $\max \left\{t: m_{t} \leq q+1\right\}+1$. 
Teorema 2.1. [7] Se A é uma K-álgebra de dimensão $n>2$, S é um conjunto gerador de A e $\left(m_{0}, \ldots, m_{s}\right)$ é a sua sequência característica. Então para todo inteiro positivo $h \leq s$ vale que $m_{h} \leq 2^{h-1}$.

Demonstração. Provaremos por indução em $h$. O caso base $h=1$ é trivial, pois $m_{1}=1 \leq 2^{0}$. Suponha que o resultado seja válido para quaisquer inteiros positivos $k$ tais que $h \leq k<s$. Vamos prová-lo para $h=k+1 \leq s$. Pelo Lema 2.5 temos que $m_{k+1}=m_{t_{1}}+m_{t_{2}}$ com $0<t_{1} \leq t_{2}<k+1$. De acordo com as hipóteses $m_{t_{1}}+m_{t_{2}} \leq 2^{t_{1}-1}+2^{t_{2}-1} \leq 2^{k-1}+2^{k-1}=2^{k}$, concluindo a prova.

Teorema 2.2. [7] Se A é uma K-álgebra com elemento identidade e dimensão $n>2$, então $l(A) \leq 2^{n-2}$.

Demonstração. Pelo Lema 2.4, se $S$ é um conjunto gerador de $A$, então $l(S)$ é igual ao último elemento da sequência característica de $S$,o qual é $\operatorname{dim} A-1=n-1$. Assim, decorre do Teorema 2.1 que $l(S)=m_{h} \leq 2^{(n-1)-1}=2^{n-2}$. Portanto, $l(A)=\max \{l(S): \mathcal{L}(S)=A\} \leq 2^{n-2}$.

Exemplo 2.9. Vamos mostrar que o limite superior obtido no Teorema 2.1 não pode ser melhorado. De fato, considere a álgebra $A$ gerada por $\left\{e_{1}, \ldots, e_{n}\right\}$, com o produto definido por: $e_{k}^{2}=e_{k+1}$, para $1 \leq k \leq n-2, e_{n-1}^{2}=0, e_{q} e_{p}=0$, para $1 \leq p \neq q \leq n-1$. Neste caso $S=\left\{e_{1}\right\}$ é um conjunto gerador tal que $l(S)=2^{n-2}$.

Encerramos esta seção destacando que as álgebras construídas no exemplo 2.9 são comutativas, o que mostra que a cota também não é menor na classe das álgebras comutativas.

\subsection{Comprimentos realizáveis}

Nesta seção, iremos nos concentrar em comprimentos que não podem ser atingidos por uma álgebra que não é unitária e que possui dimensão $n$. Por conveniência, assumiremos que os conjuntos geradores são refinados.

Pela Aplicação 2, sabemos que o comprimento nunca excede o valor de $2^{n-1}$. Durante o resto da seção assumiremos $p>2^{n-2}$, isto é, $p \in\left(2^{n-2}, 2^{n-1}-1\right]$. Note que, em uma álgebra de dimensão $n$, para que uma cadeia satisfaça $a_{2^{n-2}}<n$ devemos ter necessariamente $a_{1}=1$. Com efeito, temos que $n>a_{2^{n-2}}=a_{1}+\sum_{i=1}^{n-2}\left(a_{2^{j}}-a_{2^{j-1}}\right) \geq a_{1}+n-2$ e então $2>a_{1}$, ou seja, $a_{1}=1$.

Seja $a_{0}=0 \rightarrow a_{1}=1 \rightarrow a_{2}=2 \rightarrow \cdots \rightarrow a_{p}=n$ com $p>2^{n-2}$ uma cadeia de um conjunto gerador. Para cada $k=1,2, \ldots, n$ podemos destacar o termo de menor índice $i$ que satisfaz a igualdade $a_{i}=k$ e utilizá-lo para construir uma sequência. No caso mencionado obtemos a sequência $\left\{a_{1}, a_{2}, \ldots, a_{p}\right\}$.

Destacamos que nem toda sequência $\left\{a_{1}, a_{2}, \ldots, a_{p}\right\}$ com $p>2^{n-2}$ pode ser obtida de uma cadeia de um conjunto gerador, um exemplo trivial é a sequência $\left\{a_{1}, a_{2}, a_{8}\right\}$ cuja cadeia é dada por $a_{0}=0 \rightarrow a_{1}=1 \rightarrow a_{2}=2 \rightarrow a_{3}=2 \rightarrow a_{4}=2 \rightarrow \cdots \rightarrow 2 \rightarrow 2 \rightarrow a_{8}=3$ que viola o Lema 2.1. Desse modo, utilizamos o Lema 2.1 e o fato de que $a_{1}=1$ para estabelecer condições necessárias sobre uma sequência $\left\{a_{i_{1}}, a_{i_{2}}, \ldots, a_{i_{m}}\right\}$ para que esta tenha sido obtida de uma cadeia de um conjunto gerador.

(1) Os índices satisfazem as relações $1=i_{1}<i_{2}<\cdots<i_{m}$ e $i_{j} \leq 2\left(i_{j-1}\right)$; 
(2) A sequência não possui mais que $n$ termos;

(3) Se $j>1$, então $i_{j}=i_{q}+i_{l} \operatorname{com} l, q<j$ onde $i_{l}$ e $i_{q}$ são índices presentes na sequência.

Uma consequência importante das propriedades (1),(2) e (3) é a seguinte: Fixamos uma álgebra $A$ com dimensão $n>3$. Se $\left\{a_{i_{1}}, a_{i_{2}}, \ldots, a_{i_{m}}\right\}$ com $i_{m}>2^{n-2}$ é uma sequência obtida a partir da cadeia de um conjunto gerador $S$ e $\left(0,2^{n-1}\right]=(0,1] \cup(1,2] \cup \cdots \cup\left(2^{n-3}, 2^{n-2}\right] \cup\left(2^{n-2}, 2^{n-1}\right]$ é uma partição do intervalo $\left(0,2^{n-1}\right]$, então a sequência $\left\{a_{i_{1}}, a_{i_{2}}, \ldots, a_{i_{m}}\right\}$ satisfaz as condições $(1),(2),(3)$ e cada um dos intervalos disjuntos acima contém um único índice $i_{j} \in\left\{i_{1}, \ldots, i_{m}\right\}$.

A sequência satisfaz as condições (1),(2),(3) por construção. Vamos mostrar que cada intervalo contém pelo menos um índice. Com efeito, o índice $i_{1}=1$ pertence ao intervalo $(0,1]$ e por (1) este índice sempre está presente na cadeia. Suponha que não existe um índice pertencente ao intervalo $\left(2^{n-q}, 2^{n-q+1}\right]$ com $3 \leq q \leq n$. Tomamos o maior índice $r$ que satisfaz $r<2^{n-q}$. Por maximalidade e pelo fato de que não há índices em $\left(2^{n-q}, 2^{n-q+1}\right]$, o sucessor do índice $r$, denotado por $r^{\prime}$, deve satisfazer $r^{\prime}>2^{n-q+1}$, caso contrário, os índices da sequência seriam limitados por $2^{n-p+1} \mathrm{e}$ assim $i_{m} \leq 2^{n-p+1}$, um absurdo. Isto mostra que $r^{\prime}>2^{n-q+1}=2\left(2^{n-q}\right)>2 r$, violando a condição (3).

Suponha agora que há pelo menos dois índices no intervalo $\left(2^{n-t}, 2^{n-t+1}\right]$. A quantidade de intervalos restantes cujo extremo inferior é menor que $2^{n-t}$ na união descrita é $p+1$ onde $p=n-t$ pois precisamos incluir o intervalo $(0,1]$, isto é, são contabilizados $n-t+1$ índices. Repetimos o procedimento para contabilizar a quantidade de intervalos cujo extremo superior é maior que $2^{n-t+1}$, o que corresponde a $p^{\prime}$ onde $p^{\prime}=n-1-(n-t+1)$. Uma vez que cada intervalo contém pelo menos um índice, a sequência deve possuir pelo menos um total de $p+p^{\prime}=n-1-(n-t+$ 1) $+n-t+1=n-1$ índices que não pertencem ao intervalo $\left(2^{n-t}, 2^{n-t+1}\right]$, que possui dois índices e então a sequência possui $n-1+2=n+1$ termos, contrariando (2). Isto mostra que cada intervalo necessariamente possui um único índice pertencente à sequência. Esta discussão prova o lema a seguir.

Lema 2.7. Sejam $A$ uma álgebra com dimensão $n>2, i_{m}$ um inteiro satisfazendo $i_{m}>2^{n-2}$ e $\left\{a_{i_{1}}, a_{i_{2}}, \ldots, a_{i_{m}}\right\}$ uma sequência que satisfaz (1), (2) e (3). Então cada intervalo na forma $(0,1]$ ou $\left(2^{n-k-1}, 2^{n-k}\right]$ com $1 \leq k \leq n-1$ necessariamente contém um único índice $i_{j}$ pertencente a sequência.

Uma consequência imediata do Lema 2.7 é que o índice $i_{m}$ está limitado superiormente pela soma de um índice pertencente a $\left(2^{n-4}, 2^{n-3}\right]$ com um índice pertencente a $\left(2^{n-3}, 2^{n-2}\right]$, ou seja, $i_{m} \leq 2^{n-2}+2^{n-3}=3.2^{n-3}$ ou $i_{m}=2^{n-1}$. Isto mostra que uma álgebra de dimensão $n$ não pode ter comprimento pertencente ao intervalo $\left[3.2^{n-3}+1,2^{n-1}-1\right]$. Usaremos um raciocínio similar para extrair informações sobre os comprimentos situados no intervalo $\left(2^{n-2}, 3.2^{n-3}\right]$.

Quando uma sequência satisfaz as propriedades (1), (2), (3) dizemos que esta é uma sequência válida. Através das propriedades (1),(2) e (3) podemos determinar alguns comprimentos que não podem ser atingidos como mostra o argumento a seguir. Se não existe uma sequência válida $\left\{a_{i_{1}}, a_{i_{2}}, \ldots, a_{i_{m}}\right\}$ de $n$ termos com $i_{m}>2^{n-2}$ que satisfaz as propriedades (1),(2),(3) então não existe uma álgebra com dimensão $n$ cujo comprimento é $i_{m}$. Suponha que tal álgebra exista e fixe um conjunto gerador $S$ de comprimento $i_{m}$. Construímos a cadeia de $S$ e dela extraímos uma sequência válida que possui $n$ termos e que por construção deve satisfazer (1),(2),(3), uma 
contradição. Para provar o principal resultado desta seção precisaremos ainda do lema a seguir.

Lema 2.8. Sejam $n \in \mathbb{N} \operatorname{com} n>3$. Se $j \in \mathbb{N}$ é ímpar e satisfaz $2^{n-2}+3 \leq j \leq 2^{n-1}$, então não existe uma sequência válida de $n$ termos cujo último termo tenha índice $j$.

Demonstração. Provaremos por indução em $n$.

Caso base, $n=4$ : Observe que 7 é o único número que satisfaz as hipóteses do teorema. Suponha que $\left\{a_{1}, a_{2}, a_{i}, a_{7}\right\}$ seja uma sequência válida. Segue de (1) que $i \in\{p \in \mathbb{N}: p \leq 4 e 7 \leq 2 p\}$, isto é , $i=4$. Como a sequência $\left\{a_{1}, a_{2}, a_{4}, a_{7}\right\}$ viola a condição (3), concluímos que não há uma sequência válida.

Hipótese de indução, $n=k-1$ : Suponha que não existe uma sequência válida com $k-1$ termos cujo último termo tenha índice ímpar $j$ satisfazendo $2^{k-2}>j \geq 2^{k-3}+3$.

Passo indutivo, $n=k$ : Seja $j$ um número ímpar satisfazendo $2^{k-1}>j \geq 2^{k-2}+3$. Suponha que exista uma sequência válida $\left\{a_{1}, a_{2}, \ldots, a_{j}\right\}$ com $k$ termos. Devemos ter $j=i_{1}+i_{2}$, com $i_{1}, i_{2}<j$. Como $j$ é ímpar, podemos supor sem perda de generalidade que $i_{1}$ é ímpar. Pelo menos um dos números $i_{1}, i_{2}$ deve pertencer ao intervalo $\left(2^{k-3}, 2^{k-2}\right]$, do contrário, a soma seria limitada por $2^{k-2}$ que é menor que $2^{k-2}+3$ e pelo Lema 2.7 apenas um deles pode pertencer a este intervalo.

Agora, vamos analisar os casos $i_{1}>i_{2}$ e $i_{2}>i_{1}$. Se $i_{1}>i_{2}$ então temos que $i_{1} \in\left(2^{k-3}, 2^{k-2}\right]$. Nesse caso, deve haver uma sequência válida $\left\{a_{1}, \ldots, a_{i_{2}}, \ldots, a_{i_{1}}\right\}$ com $k-1$ termos, ou seja, uma sequência válida de $k-1$ termos cujo último termo possui índice ímpar $i_{1}>2^{k-3}$ e pela hipótese de indução concluímos que $i_{1}<2^{k-3}+3$. Podemos supor que $i_{2} \in\left(2^{k-4}, 2^{k-3}\right]$ pois se $i_{2} \leq 2^{k-4}$ então $i_{1}+$ $i_{2}<\left(2^{k-3}+3\right)+2^{k-4}<2^{n-2}+3 \leq j$, uma contradição. Trataremos então do caso em que $i_{1}>i_{2}$ com $i_{1} \in\left(2^{k-3}, 2^{k-2}\right]$ e $i_{2} \in\left(2^{k-4}, 2^{k-3}\right]$. Novamente, deve haver uma sequência válida $\left\{a_{1}, \ldots, a_{i_{2}}, a_{i_{1}}\right\}$ com $k-1$ termos e pela hipótese de indução obtemos $i_{1}<2^{k-3}+3$, consequentemente $i_{1}+i_{2}<$ $2^{k-3}+3+2^{k-3}=2^{k-2}+3 \leq j$, absurdo. Isto mostra que devemos ter $i_{2}>i_{1}$. Novamente aplicando a hipótese de indução em $i_{1}$ obtemos que este deve ser limitado por $2^{n-4}+3$ mas pertence ao intervalo $\left(2^{n-4}, 2^{n-3}\right]$, isto é, $i_{1}=2^{n-4}+1$. Como a sequência deve respeitar a propriedade (1) o índice $i_{2}$ está limitado pelo valor $2\left(2^{n-4}+1\right)$ e então $i_{1}+i_{2} \leq 2^{n-4}+1+2\left(2^{n-4}+1\right)=3.2^{n-4}+3<$ $2^{n-2}+3 \leq j$, novamente um absurdo. Isto mostra que nenhuma sequência pode apresentar um índice ímpar superior a $2^{n-2}+3$ como proposto e o lema está provado.

Proposição 2.5. Seja $A$ uma $K$-álgebra de dimensão $n>3$. Então $l(A) \notin\left[3 \cdot 2^{n-3}+1,2^{n-1}-1\right]$. Além disso, se $l(A) \in\left[2^{n-2}+3,3.2^{n-3}\right]$ então $l(A)$ não pode ser um número ímpar.

Demonstração. Pelo Lema 2.7 concluímos que $l(A) \notin\left[3.2^{n-3}+1,2^{n-1}-1\right]$. Suponha agora que existe uma álgebra de dimensão $n>3$ que possui um conjunto gerador $S$ cujo comprimento é o número ímpar $j$ que satisfaz $2^{n-2}+3 \leq j \leq 3.2^{n-3}$. Extraímos da cadeia de $S$ uma sequência válida de $n$ termos cujo primeiro termo é 1 e o último é $j$, contrariando o Lema 2.8 . 


\section{Capítulo 3}

\section{O comprimento de álgebras de dimensão baixa}

\subsection{Classificação de álgebras pelo comprimento}

\subsubsection{Nota histórica}

A relevância da classificação de objetos em álgebra está refletida na existência de teoremas que qualificam grupos como, por exemplo, os Teoremas de Sylow e o Teorema fundamental dos grupos abelianos finitos, e se estende até a classificação de módulos artinianos e noetherianos. A caracterização de álgebras, assim como de qualquer outra estrutura, requer o uso de invariantes tais como índice de nilpotência e dimensão. No entanto, propriedades como associatividade e comutatividade também podem ser levadas em consideração. Os trabalhos [12] e [16] ilustram a importância do estudo da nilpotência na classificação das álgebras de Lie e Malcev e nas próximas seções nos concentraremos na associatividade como instrumento de estudo das álgebras.

Classificar as álgebras associativas de dimensão arbitrária sobre um corpo qualquer é ainda um problema em aberto, apesar do estudo deste problema ser datado de 1881 quando Peirce [21] descreveu álgebras associativas de dimensão baixa exibindo uma imensa quantidade de casos a serem tratados, fato este que tem análogo na determinação do comprimento de uma álgebra não associativa. Em particular, as possibilidades para os valores das potências de um elemento aumenta de forma não linear. Para dimensões menores que três é sabido que as álgebras alternativas coincidem com as associativas porém, isto não ocorre para dimensões maiores. $O$ trabalho de Goodaire e Zhou em [6] é definitivo na classificação de álgebras alternativas de dimensão menor que 6 sobre um corpo perfeito, admitindo ainda, extensões para corpos mais gerais, decorrentes do trabalho de classificação de álgebras nilpotentes feito por Badalov em [2].

Os próximos parágrafos oferecem demonstrações construtivas de propriedades estruturais de uma álgebra qualquer para que esta tenha um comprimento fixado a priori. O objetivo deste capítulo é prover um apanhado de resultados gerais sobre álgebras de comprimentos fixados e munir o leitor dos resultados necessários para os próximos capítulos. 


\subsubsection{Caracterizações parciais}

Novamente denotaremos por $A$ uma K-álgebra arbitrária de dimensão finita e $S$ um conjunto gerador refinado de $A$. Começaremos esta seção com o seguinte resultado:

Proposição 3.1. Se $A$ é uma $K$-álgebra com dimensão $n \geq 2$, então são válidas as seguintes afirmações:

1. Se $A$ não é unitária, então $l(A)=1$ se, e somente se, para quaisquer $x, y \in A$ vale a relação $x y \in\langle x, y\rangle$;

2. Se $A$ é unitária, então $l(A)=1$ se, e somente se, para quaisquer $x, y \in A$, temos que $x y \in$ $\langle 1, x, y\rangle$.

Demonstração. As demonstrações dos itens 1 e 2 são análogas, por isso, faremos apenas a do item 1. Suponhamos que $l(A)=1$. Suponha que existem elementos $x, y \in A$ tais que $x y \notin\langle x, y\rangle$, então fixamos o conjunto $\{x, y\}$. Por um lado, se o conjunto $\{x, y\}$ é linearmente dependente, podemos escrever $x=k y$ para algum $k \in K$, o que implica em $\langle x, y\rangle=\langle x\rangle$ e nesse caso $x^{2} \notin\langle x\rangle$, então o conjunto $\left\{x, x^{2}\right\}$ está contido em uma base $B$ de $A$ e o conjunto $S=B \backslash\left\{x^{2}\right\}$ é gerador de comprimento 2 , contradizendo a hipótese $l(A)=1$. Por outro lado, se o conjunto $\{x, y\}$ é linearmente independente, então o conjunto $\{x, y, x y\}$ pode ser estendido para uma base $B^{\prime}$ de $A$. O conjunto $S=B^{\prime} \backslash\{x y\}$ é gerador e tem comprimento 2, novamente contradizendo a hipótese. Reciprocamente se $x y \in\langle x, y\rangle$ para quaisquer $x, y \in A$, então $\mathcal{L}_{1}(S)=\mathcal{L}_{2}(S)$ para qualquer conjunto gerador $S$, o que implica em $l(A)=1$.

\subsubsection{Dimensão 1}

Se $A$ é uma $K$-álgebra de dimensão 1 , as únicas possibilidades de cadeias para um conjunto gerador são $a_{0}=1$ se a álgebra for unitária e $0 \rightarrow 1$ se a álgebra não for unitária. Desse modo, $A$ possui comprimento 1 se não for unitária ou comprimento 0 se for unitária.

\subsubsection{Dimensão 2}

O objetivo desta subseção é demonstrar o seguinte resultado:

Teorema 3.1. Se A é uma K-álgebra de dimensão 2, então $l(A)=2$ se, e somente se, A não é unitária e existe $x \in A$ tal que $\left\{x, x^{2}\right\}$ é base de A.

Se $A$ é uma álgebra unitária e $S$ é um conjunto gerador, então existe $x \in S$ tal que o conjunto $\left\{1_{A}, x\right\}$ é linearmente independente, do contrário, pelo Corolário 2.1, $S$ não seria gerador. Isso mostra que se $A$ é unitária e tem dimensão 2 , todo conjunto gerador possui comprimento 1 . Seja então $A$ uma álgebra de dimensão $2 \operatorname{com} l(A)=2$. Fixando um conjunto gerador $S$ tal que $l(S)=2$. Assim, $0 \rightarrow 1 \rightarrow 2$ é a cadeia de $S$. Desse modo, existe $x \neq 0$ tal que $\mathcal{L}_{1}(S)=\langle x\rangle$ e $\mathcal{L}_{2}(S)=\left\langle x, x^{2}\right\rangle=A$. Portanto, $\left\{x, x^{2}\right\}$ é uma base de $A$. Reciprocamente, suponha que exista $x \in A$ tal que $\left\{x, x^{2}\right\}$ seja uma base para $A$. Considerando $S=\{x\}$, temos que $l(S)=2$, pois $\mathcal{L}_{1}(S) \neq A$ e $\mathcal{L}_{2}(S)=A$. Assim $l(A) \geq 2$. Por outro lado, pelo Lema $2.1, l(S) \leq 2$ para qualquer conjunto gerador refinado $S$ de $A$. Portanto $l(A)=2$. 


\subsubsection{Dimensão 3}

Neste caso, decorre do Teorema 2.2 que $l(A) \leq 4$ se $A$ não for unitária, e $l(A) \leq 2$ se $A$ for unitária.

Exemplo 3.1. (Álgebra de dimensão 3 e comprimento 4).

Considere a álgebra $A$ com base $\left\{e_{1}, e_{2}, e_{3}\right\}$ cuja tabela de multiplicação é dada a seguir:

\begin{tabular}{|l|l|l|l|}
\hline$*$ & $e_{1}$ & $e_{2}$ & $e_{3}$ \\
\hline$e_{1}$ & $e_{2}$ & $e_{1}$ & $e_{3}$ \\
\hline$e_{2}$ & $e_{1}$ & $e_{3}$ & $e_{1}$ \\
\hline$e_{3}$ & $e_{3}$ & $e_{1}$ & $e_{3}$ \\
\hline
\end{tabular}

Considerando o conjunto $S=\left\{e_{1}\right\}$ temos $\mathcal{L}_{0}(S)=\{0\}, \mathcal{L}_{1}(S)=\left\langle e_{1}\right\rangle, \mathcal{L}_{2}(S)=\left\langle e_{1}, e_{2}\right\rangle=\mathcal{L}_{3}(S)$ e $\mathcal{L}_{4}(S)=\left\langle e_{1}, e_{2}, e_{3}\right\rangle=A$. Desse modo $l(A)=4$.

Agora, seja $A$ uma álgebra que não é unitária. Supomos inicialmente que $l(A)=4$. Se $S$ for um conjunto gerador refinado de $A$ tal que $l(S)=4$, vamos determinar as possíveis cadeias para $S$. A princípio já sabemos que $\mathcal{L}_{4}(S)=A$ e que $\mathcal{L}_{3}(S) \neq A$. Desse modo, se $a_{0}=0 \rightarrow a_{1} \rightarrow a_{2} \rightarrow a_{3} \rightarrow a_{4}$ é uma cadeia de $S$ temos que $a_{4}=3$ mas $a_{3}<3$. Note que se $a_{1} \geq 2$, então $a_{2} \geq 3$ pelo Lema 2.1, o que é uma contradição pois $a_{3}<3$. Portanto $a_{1}=1$. Novamente pelo Lema 2.1 sabemos que $a_{2}>a_{1}$. Assim $a_{2}=2$, o que nos permite concluir que $a_{3}=2$. Logo $0 \rightarrow 1 \rightarrow 2 \rightarrow 2 \rightarrow 3$, cuja sequência característica é $(0,1,2,4)$, é a única cadeia possível para $S$. Desse modo, existe $x \in S$ tal que $\mathcal{L}_{1}(S)=\langle x\rangle, \mathcal{L}_{2}(S)=\mathcal{L}_{3}(S)=\left\langle x, x^{2}\right\rangle$ e $\mathcal{L}_{4}(S)=\left\langle x, x^{2}, x^{2} x^{2}\right\rangle=A$ e consequentemente $\left\{x, x^{2}, x^{2} x^{2}\right\}$ é uma base de $A$. Reciprocamente, se $A$ possui um conjunto gerador $S$ cuja sequência característica é $(0,1,2,4)$, então $\operatorname{dim} \mathcal{L}_{4}(S)-\operatorname{dim} \mathcal{L}_{3}(S)=1$, portanto $l(S)=4=l(A)$.

Suponhamos que $l(A)=3$. Assim, existe um conjunto gerador refinado $S$ tal que $l(S)=3$. Desse modo, $\mathcal{L}_{3}(S)=A$ e $\mathcal{L}_{2}(S) \neq A$. Se $a_{0}=0 \rightarrow a_{1} \rightarrow a_{2} \rightarrow a_{3}=3$ é uma cadeia de $S$, então como $a_{2}<3$ e $a_{1}<a_{2}$ pelo Lema 2.1, as únicas possibilidades são $a_{1}=1$ e $a_{2}=2$. Portanto $0 \rightarrow 1 \rightarrow 2 \rightarrow 3$ é a única cadeia possível para $S$. Consequentemente existe $x \in A$ tal que $\mathcal{L}_{1}(S)=\langle x\rangle, \mathcal{L}_{2}(S)=\left\langle x, x^{2}\right\rangle$, e $\mathcal{L}_{3}(S)=\left\langle x, x^{2}, x^{2} x\right\rangle$ ou $\mathcal{L}_{3}(S)=\left\langle x, x^{2}, x x^{2}\right\rangle$ (isto é, $x^{2} x=x x^{2}$ ou $x^{2} x \in \mathcal{L}_{2}(S)$ e $x x^{2} \notin \mathcal{L}_{2}(S)$ ou $x^{2} x \notin \mathcal{L}_{2}(S)$ e $x x^{2} \in \mathcal{L}_{2}(S)$. Logo, existe $x \in A$ tal que um dos conjuntos $\left\{x, x^{2}, x^{2} x\right\}$ ou $\left\{x, x^{2}, x x^{2}\right\}$ é uma base de $A$. Pode-se verificar que a recíproca é falsa, considerando-se o conjunto $S=\left\{e_{2}\right\}$ no Exemplo 3.1.

Supomos que $A$ seja uma álgebra unitária tal que $l(A)=2$. Assim, existe um conjunto gerador refinado $S$ de $A$ tal que $l(S)=2$. A única cadeia possível para $S$ é $1 \rightarrow 2 \rightarrow 3$, pois $A$ é unitária e $\operatorname{dim} \mathcal{L}_{1}(S)>\operatorname{dim} \mathcal{L}_{0}(S)$ e $\operatorname{dim} \mathcal{L}_{2}(S)>\operatorname{dim} \mathcal{L}_{1}(S)$ pelo Lema 2.1. Desse modo, existe $x \in S$ tal que $\left\{1, x, x^{2}\right\}$ é uma base de $A$. Reciprocamente, se existir $x \in A$ tal que $\left\{1, x, x^{2}\right\}$ é uma base para $A$, então $S=\{x\}$ é um conjunto gerador tal que $l(S)=2$, o que implica $l(A) \geq 2$. Portanto, $l(A)=2$.

Por fim, assumimos que $l(A)=2$ e que $A$ não possui identidade. Existe um conjunto gerador refinado $S$ tal que $l(S)=2$. Desse modo, $\mathcal{L}_{2}(S)=A$ mas $\mathcal{L}_{1}(S) \neq A$. Se $a_{0}=0 \rightarrow a_{1} \rightarrow a_{2}=3$ 
é uma cadeia de $S$, então como $a_{0}<a_{1}<a_{2}$, devemos ter $a_{1}=2$ pois se $a_{1}=1$ teríamos que $a_{2} \leq 2$. Portanto, $0 \rightarrow 2 \rightarrow 3$ é a única cadeia possível para $S$. Assim, existem $x, y \in A$ tais que $\mathcal{L}_{1}(S)=\langle x, y\rangle, \mathcal{L}_{2}(S)=\left\langle x, x^{2}, y\right\rangle$ ou $\mathcal{L}_{2}(S)=\langle x, y, x y\rangle$. Portanto, existem elementos $x, y \in A$ tais que um dos conjuntos $\left\{x, y, x^{2}\right\}$ ou $\{x, y, x y\}$ é uma base de $A$. A recíproca é falsa como pode ser comprovado considerando-se $S=\left\{e_{1}, e_{3}\right\}$ e $S=\left\{e_{2}, e_{3}\right\}$ no Exemplo 3.1, respectivamente.

A discussão acima demonstra o resultado a seguir.

Teorema 3.2. Seja A uma K-álgebra de dimensão 3. São verdadeiras as seguintes afirmações:

- A tem comprimento 4 se, e somente se, não possui identidade e existe um conjunto gerador $S$ cuja sequência característica é $(0,1,2,4)$. Neste caso, existe $x \in A$ tal que $\left\{x, x^{2},(x x)(x x)\right\}$ é base de $A$;

- Se A tem comprimento 3, então A não possui identidade e existe $x \in A$ tal que um dos conjuntos $\left\{x, x^{2}, x x^{2}\right\}$ ou $\left\{x, x^{2}, x^{2} x\right\}$ é base de $A$;

- Se A não possui identidade e tem comprimento 2, então A possui elementos $x$ e y tais que um dos conjuntos $\{x, y, x y\}$ ou $\left\{x, y, x^{2}\right\}$ é base de A;

- Se A é unitária, então tem comprimento 2 se, e somente se, existe $x \in A$ tal que $\left\{1, x, x^{2}\right\}$ é base de A.

\subsubsection{Dimensão 4}

O objetivo desta subseção é demonstrar o seguinte resultado:

Teorema 3.3. Seja A uma K-álgebra de dimensão 4. São verdadeiras as seguintes afirmações:

- A tem comprimento 8 se, e somente se, A não possui identidade e existe um conjunto gerador $S$ cuja sequência característica é $(0,1,2,4,8)$. Neste caso, existe $x \in A$ tal que $\left\{x, x^{2},\left(x^{2}\right)^{2},\left(x^{2}\right)^{2}\left(x^{2}\right)^{2}\right\}$ é base de A;

- A não pode ter comprimento 7;

- Se A tem comprimento 6 então A não possui identidade e existe $x \in A$ tal que um dos conjuntos $\left\{x, x^{2},\left(x^{2}\right)^{2}, x^{2}\left(x^{2}\right)^{2}\right\} ;\left\{x, x^{2},\left(x^{2}\right)^{2},\left(x^{2}\right)^{2} x^{2}\right\} ;\left\{x, x^{2}, x x^{2},\left(x x^{2}\right)^{2}\right\}$ ou $\left\{x, x^{2}, x^{2} x,\left(x^{2} x\right)^{2}\right\}$ é base de A;

- Se A tem comprimento 5, então A não possui identidade e existe $x \in A$ tal que um dos conjuntos $\left\{x, x^{2}, x x^{2}, x^{2}\left(x x^{2}\right)\right\} ;\left\{x, x^{2}, x x^{2},\left(x x^{2}\right) x^{2}\right\} ;\left\{x, x^{2}, x^{2} x, x^{2}\left(x^{2} x\right)\right\} ;\left\{x, x^{2}, x^{2} x,\left(x^{2} x\right) x^{2}\right\}$ $\left\{x, x^{2}, x^{2} x^{2},\left(x^{2} x^{2}\right) x\right\}$ ou $\left\{x, x^{2}, x^{2} x^{2}, x\left(x^{2} x^{2}\right)\right\}$ é base de A;

- Se A é unitária, então A tem comprimento 4 se, e somente se, existe um conjunto gerador $S$ que tem como sequência característica $(0,1,2,4)$ e, nesse caso, possui um elemento $x$ tal que o conjunto $\left\{1, x, x^{2}, x^{2} x^{2}\right\}$ é base de A;

- Se A não possui identidade, então tem comprimento 4 se existe um elemento $x \in A$ tal que um dos conjuntos

$\left\{x, x^{2}, x x^{2}, x\left(x x^{2}\right)\right\} ;\left\{x, x^{2}, x x^{2},\left(x x^{2}\right) x\right\} ;\left\{x, x^{2}, x^{2} x, x\left(x^{2} x\right)\right\} ;\left\{x, x^{2}, x^{2} x,\left(x^{2} x\right) x\right\}$ é base de $A$ ou elementos $x, y \in A$ tal que um dos conjuntos $\left\{x, y, x^{2},\left(x^{2}\right)^{2}\right\}$ ou $\left\{x, y, x y,(x y)^{2}\right\}$ é base de $A$; 
- Se A é unitária, então A tem comprimento 3 se existe um elemento $x \in A$ tal que um dos conjuntos $\left\{1, x, x^{2}, x(x x)\right\}$ ou $\left\{1, x, x^{2},(x x) x\right\}$ é base de $A$;

- Se A não possui identidade, então tem comprimento 3 se existe um elemento $x \in A$ tal que $\left\{x, x^{2}, x x^{2}, x^{2} x\right\}$ é base de A ou elementos $x, y \in A$ tais que um dos conjuntos $\left\{x, y, x^{2}, x^{2} x\right\} ;\left\{x, y, x^{2}, x x^{2}\right\} ;\left\{x, y, x^{2}, x^{2} y\right\}$; $\left\{x, y, x^{2}, y x^{2}\right\} ;\{x, y, x y, x(x y)\} ;\{x, y, x y,(x y) x\} ;\{x, y, x y, y(x y)\}$ ou $\{x, y, x y,(x y) y\}$ é base de A;

- Se A não possui identidade, então tem comprimento 2 se existem elementos $x, y, z \in A$ tal que um conjunto na forma $\left\{x, y, z, x^{2}\right\} ;\{x, y, z, x y\} ;\{x, y, z, x z\}$ ou $\{x, y, z, y z\}$ é uma base de A ou elementos $x, y \in A$ tais que um conjunto na forma $\left\{x, y, x^{2}, y^{2}\right\} ;\left\{x, y, x^{2}, x y\right\}$ ou $\left\{x, y, x^{2}, y x\right\}$ é uma base de A;

- Se A é unitária, então tem comprimento 2 se existem elementos $x$ e y tais que um dos conjuntos $\{1, x, y, x y\}$ ou $\left\{1, x, y, x^{2}\right\}$ é base de $A$.

Antes de demonstrar o Teorema 8, provaremos o seguinte resultado auxiliar.

Lema 3.1. Seja $A$ uma $K$-álgebra de dimensão 4. São verdadeiras as seguintes afirmações:

- $l(A)=8$ se, e somente se, existe um conjunto gerador $S$ que tem a sequência característica $(0,1,2,4,8)$;

- Se $l(A)=6$, então existe um conjunto gerador $S$ que tem como sequência característica $(0,1,2,4,6)$ ou $(0,1,2,3,6)$;

- Se $l(A)=5$, então existe um conjunto gerador $S$ que tem como sequência característica $(0,1,2,3,5)$ ou $(0,1,2,4,5)$;

- Se $l(A)=4$ e $A$ não é unitária, então existe um conjunto gerador $S$ que tem como sequência característica $(0,1,2,3,4)$ ou $(0,1,1,2,4)$;

- Se $A$ é unitária, então $l(A)=4$ se, e somente se, existe um conjunto gerador $S$ que tem como sequência característica $(0,1,2,4)$;

- Se $l(A)=3$ e $A$ não é unitária, então existe um conjunto gerador $S$ que tem como sequência característica $(0,1,1,2,3)$ ou $(0,1,2,3,3)$;

- Se $l(A)=3$ e $A$ é unitária, então existe um conjunto gerador $S$ que tem como sequência característica $(0,1,2,3)$;

- Se $l(A)=2$ e $A$ não é unitária, então existe um conjunto gerador $S$ que tem como sequência característica $(0,1,1,1,2)$ ou $(0,1,1,2,2)$;

- Se $l(A)=2$ e $A$ é unitária, então existe um conjunto gerador $S$ que tem como sequência característica $(0,1,1,2)$.

Demonstração. Pelo Teorema 2.2 as únicas álgebras que podem atingir comprimentos maiores que 4 são aquelas que não possuem identidade. Supomos que $l(A)=8$. Neste caso, existe um conjunto gerador refinado $S$ que tem a cadeia $0=a_{0} \rightarrow a_{1} \rightarrow \cdots \rightarrow a_{8}=4$. Pelo Lema 2.1 concluímos 
que $a_{1}=1, a_{2}=2$ e $a_{4}=3$. Desse modo, a única possibilidade para cadeia de $S$ é $0 \rightarrow 1 \rightarrow 2 \rightarrow 2 \rightarrow$ $3 \rightarrow 3 \rightarrow 3 \rightarrow 3 \rightarrow 4$, cuja sequência característica correspondente é $(0,1,2,4,8)$. Reciprocamente, se existir um conjunto gerador $S$ cuja sequência característica é dada por $(0,1,2,4,8)$, então, pela definição de sequência característica temos que $\operatorname{dim} \mathcal{L}_{8}(S)-\operatorname{dim} \mathcal{L}_{7}(S)=1$ e portanto $l(S)=8$, além disso, $S$ contém apenas um elemento.

Se $l(A)=6$, existe um conjunto gerador refinado $S$ com cadeia $0=a_{0} \rightarrow a_{1} \rightarrow \cdots \rightarrow a_{6}=4$. Utilizando o Lema 2.1 como no parágrafo anterior concluímos que $a_{1}=1$ e $a_{2}=2$. Se $a_{3}=2$ então $a_{4}=a_{5}=3$ pois $3 \leq a_{4} \leq a_{5}<4$ e obtemos a cadeia $0 \rightarrow 1 \rightarrow 2 \rightarrow 2 \rightarrow 3 \rightarrow 3 \rightarrow 4$. Por outro lado, se $a_{3}=3$ então $3=a_{3} \leq a_{4} \leq a_{5} \leq 4$ e obtemos a cadeia $0 \rightarrow 1 \rightarrow 2 \rightarrow 3 \rightarrow 3 \rightarrow 3 \rightarrow 4$.

Para o caso $l(A)=5$, prosseguimos da mesma forma que no caso anterior e a cadeia de um conjunto gerador $S$ deve ser da forma $0 \rightarrow 1 \rightarrow 2 \rightarrow a_{3} \rightarrow a_{4} \rightarrow a_{5}=4$. Novamente, se $a_{3}=2$ teremos $a_{4}=3$, e quando $a_{3}=3$ teremos $a_{4}=3$. Obtemos assim as cadeias $0 \rightarrow 1 \rightarrow 2 \rightarrow 2 \rightarrow 3 \rightarrow 4 \mathrm{e}$ $0 \rightarrow 1 \rightarrow 2 \rightarrow 3 \rightarrow 3 \rightarrow 4$, respectivamente.

Supomos agora que $l(A)=4$ e que $A$ não é unitária. Assim, existe um conjunto gerador $S$ cuja cadeia é $0 \rightarrow a_{1} \rightarrow a_{2} \rightarrow a_{3} \rightarrow a_{4}=4$. Se $a_{1}=1$ então $2 \leq a_{2} \leq a_{3}<4$. Desse modo, se fosse $a_{2}=2=a_{3}$ haveria apenas uma palavra irredutível de comprimento quatro, impossibilitando $a_{4}=4$. Assim obtemos a cadeia $0 \rightarrow 1 \rightarrow 2 \rightarrow 3 \rightarrow 4$. Se $a_{1}=2$ então $a_{2}=a_{3}=3$ e obtemos a cadeia $0 \rightarrow 2 \rightarrow 3 \rightarrow 3 \rightarrow 4$. Por outro lado, se $l(A)=4$ e $A$ é unitária, um conjunto gerador refinado $S$ deve ter como cadeia $1 \rightarrow a_{1} \rightarrow a_{2} \rightarrow a_{3} \rightarrow a_{4}=4$. Segue do Lema 2.1 que $1 \rightarrow 2 \rightarrow 3 \rightarrow 3 \rightarrow 4$ é a cadeia de $S$. Reciprocamente, se existe um conjunto gerador cuja sequência característica é dada por $(0,1,2,4)$, devemos ter que $\operatorname{dim} \mathcal{L}_{4}(S)-\operatorname{dim} \mathcal{L}_{3}=1$, o que implica em $4=l(S)=l(A)$.

Quando $l(A)=3$ e $A$ é unitária aplicamos novamente o Lema 2.1 que nos fornece a única cadeia possível para um conjunto gerador, a saber $1 \rightarrow 2 \rightarrow 3 \rightarrow 4$. Analogamente, se $A$ não é unitária e tem comprimento 3 , um conjunto gerador tem por cadeia $0 \rightarrow a_{1} \rightarrow a_{2} \rightarrow a_{3}=4$. Desse modo, se $a_{1}=2$, devemos ter $a_{2}=3$; e se $a_{1}=1$, como não é possível produzir duas palavras irredutíveis de comprimento 2 , segue que $a_{2}=2$. Obtemos assim, as cadeias $0 \rightarrow 2 \rightarrow 3 \rightarrow 4$ e $0 \rightarrow 1 \rightarrow 2 \rightarrow 4$.

Finalmente, o caso em que $A$ tem comprimento dois e não é unitária. Prosseguimos do mesmo modo que no parágrafo anterior e concluímos que as únicas cadeias possíveis são dadas por $0 \rightarrow 3 \rightarrow 4$ e $0 \rightarrow 2 \rightarrow 4$, enquanto para o caso unitário a única cadeia possível é dada por $1 \rightarrow 3 \rightarrow 4$.

Utilizamos agora o Lema 3.1 para determinar as possíveis bases para cada álgebra mencionada no Teorema 3.3. Analisamos também a reciprocidade de cada afirmação e ilustramos com contraexemplos.

Se $A$ é uma álgebra de comprimento 8 então $A$ possui um conjunto gerador com uma sequência característica da forma $(0,1,2,4,8)$. Além disso, se $A$ fosse unitária teríamos que $l(A) \leq$ 4. Reciprocamente, se existe um conjunto gerador cuja sequência característica é $(0,1,2,4,8)$, o Lema 3.1 garante que $l(A)=8$. 
Exemplo 3.2. Considere a álgebra $A$ com base $\left\{e_{1}, e_{2}, e_{3}, e_{4}\right\}$ e tabela de multiplicação dada por:

\begin{tabular}{|c|c|c|c|c|}
\hline$*$ & $e_{1}$ & $e_{2}$ & $e_{3}$ & $e_{4}$ \\
\hline$e_{1}$ & $e_{2}$ & 0 & 0 & 0 \\
\hline$e_{2}$ & 0 & $e_{3}$ & $e_{1}$ & 0 \\
\hline$e_{3}$ & 0 & $e_{1}$ & $e_{4}$ & $e_{1}$ \\
\hline$e_{4}$ & 0 & 0 & $e_{1}$ & 0 \\
\hline
\end{tabular}

Fixando o subconjunto $S=\left\{e_{1}\right\}$ temos que $\mathcal{L}_{1}(S)=\left\langle e_{1}\right\rangle, \mathcal{L}_{2}(S)=\mathcal{L}_{3}(S)=\left\langle e_{1}, e_{2}\right\rangle, \mathcal{L}_{4}(S)=$ $\mathcal{L}_{5}(S)=\mathcal{L}_{6}(S)=\mathcal{L}_{7}(S)=\left\langle e_{1}, e_{2}, e_{3}\right\rangle, \mathcal{L}_{8}(S)=\left\langle e_{1}, e_{2}, e_{3}, e_{4}\right\rangle=A$, então vale que $l(A)=8$.

Considerando agora uma álgebra de comprimento 7, seguimos o mesmo raciocínio empregado na demonstração do Lema 3.1 e concluímos que um conjunto gerador tem como cadeia $0 \rightarrow 1 \rightarrow 2 \rightarrow a_{3} \rightarrow \ldots \rightarrow a_{7}=4$. Por um lado, se $a_{3}=2$, então devemos ter $a_{4}=a_{5}=a_{6}=3$ e $a_{7}=4, \mathrm{o}$ que é impossível, pois se $A$ não possui palavras irredutíveis de comprimentos 3,4,5 e 6, então $A$ também não possui um palavra irredutível de comprimento 7 . Se $a_{3}=3$ e $a_{6}=3$, então pelo Lema $3.1, a_{7}$ não seria 4 , o que é uma contradição. Desse modo, se $a_{3}=3$, então $a_{6}>3$, o que também é uma contradição, pois $l(A)=7$. Portanto $a_{3} \neq 3$. Isto mostra que não há uma álgebra de dimensão 4 que tenha comprimento 7 .

Tomamos agora uma álgebra de comprimento 6, pelo Lema 3.1 existe um conjunto gerador que possui sequência característica dada por $(0,1,2,4,6)$ ou $(0,1,2,3,6)$ e, nesse caso, sabemos que existe $x \in A$ tal que $\left\{x, x^{2},\left(x^{2}\right)^{2}, x^{2}\left(x^{2}\right)^{2}\right\},\left\{x, x^{2},\left(x^{2}\right)^{2},\left(x^{2}\right)^{2} x^{2}\right\},\left\{x, x^{2}, x x^{2},\left(x x^{2}\right)^{2}\right\}$ ou $\left\{x, x^{2}, x^{2} x,\left(x^{2} x\right)^{2}\right\}$ é base de $A$. Cada uma das condições da recíproca é satisfeita por pelo menos um dos conjuntos $S=\left\{e_{2}\right\}$ e $T=\left\{e_{3}\right\}$ na álgebra do Exemplo 3.2, no entanto, vale a igualdade $l(A)=8$.

Exemplo 3.3. Considere a álgebra $A$ com base $\left\{e_{1}, e_{2}, e_{3}, e_{4}\right\}$ e tabela de multiplicação dada por:

\begin{tabular}{|c|c|c|c|c|}
\hline$*$ & $e_{1}$ & $e_{2}$ & $e_{3}$ & $e_{4}$ \\
\hline$e_{1}$ & $e_{2}$ & 0 & 0 & 0 \\
\hline$e_{2}$ & 0 & $e_{3}$ & $e_{4}$ & 0 \\
\hline$e_{3}$ & 0 & $e_{4}$ & $e_{1}$ & $e_{1}$ \\
\hline$e_{4}$ & 0 & 0 & $e_{1}$ & $e_{2}$ \\
\hline
\end{tabular}

Fixando $S=\left\{e_{1}\right\}$ temos as relações $\mathcal{L}_{1}(S)=\left\langle e_{1}\right\rangle, \mathcal{L}_{2}(S)=\mathcal{L}_{3}(S)=\left\langle e_{1}, e_{2}\right\rangle, \mathcal{L}_{4}(S)=\mathcal{L}_{5}(S)=$ $\left\langle e_{1}, e_{2}, e_{3}\right\rangle, \mathcal{L}_{6}(S)=\left\langle e_{1}, e_{2}, e_{3}, e_{4}\right\rangle=A$, o que implica em $l(A) \geq 6$.

Se $l(A)=5$, existe $x \in A$ tal que um dos conjuntos $\left\{x, x^{2}, x x^{2}, x^{2}\left(x x^{2}\right)\right\},\left\{x, x^{2}, x x^{2},\left(x x^{2}\right) x^{2}\right\}$, $\left\{x, x^{2}, x^{2} x, x^{2}\left(x^{2} x\right)\right\},\left\{x, x^{2}, x^{2} x,\left(x^{2} x\right) x^{2}\right\},\left\{x, x^{2}, x^{2} x^{2},\left(x^{2} x^{2}\right) x\right\}$ ou $\left\{x, x^{2}, x^{2} x^{2}, x\left(x^{2} x^{2}\right)\right\}$ é base de $A$. A recíproca é falsa. Com efeito, cada uma das condições da recíproca é satisfeita por pelo menos um dos conjuntos $S=\left\{e_{2}\right\}$ e $U=\left\{e_{4}\right\}$ na álgebra do Exemplo 3.3, no entanto, vale a desigualdade $l(A) \geq 6>5$. 
Exemplo 3.4. Considere a álgebra $A$ com base $\left\{1_{A}, e_{1}, e_{2}, e_{3}\right\}$ cuja tabela de multiplicação é dada a seguir:

\begin{tabular}{|c|c|c|c|c|}
\hline$*$ & $1_{A}$ & $e_{1}$ & $e_{2}$ & $e_{3}$ \\
\hline $1_{A}$ & $1_{A}$ & $e_{1}$ & $e_{2}$ & $e_{3}$ \\
\hline$e_{1}$ & $e_{1}$ & $e_{2}$ & $e_{3}$ & 0 \\
\hline$e_{2}$ & $e_{2}$ & $e_{3}$ & $e_{3}$ & 0 \\
\hline$e_{3}$ & $e_{3}$ & 0 & 0 & 0 \\
\hline
\end{tabular}

Nesse caso, o conjunto $\left\{1_{A}, e_{1}, e_{1}^{2}, e_{1}^{2} e_{1}^{2}\right\}$ é base mas $l(A)=3$.

Se $A$ é uma álgebra que não é unitária e tem comprimento 4 , então existe $x \in A$ tal que um dos conjuntos $\left\{x, x^{2}, x x^{2}, x\left(x x^{2}\right)\right\} ;\left\{x, x^{2}, x x^{2},\left(x x^{2}\right) x\right\} ;\left\{x, x^{2}, x^{2} x, x\left(x^{2} x\right)\right\}$ ou $\left\{x, x^{2}, x^{2} x,\left(x^{2} x\right) x\right\}$ é base enquanto para o segundo caso, existem vetores $x, y \in A$ tal que um dos conjuntos $\left\{x, y, x^{2},\left(x^{2}\right)^{2}\right\}$ ou $\left\{x, y, x y,(x y)^{2}\right\}$ é base de $A$. A recíproca é falsa e para verificação é suficiente considerar os conjuntos $S=\left\{e_{2}\right\}, T=\left\{e_{1}, e_{4}\right\}$ e $U=\left\{e_{2}, e_{3}\right\}$ na álgebra do Exemplo 3.6. Para o caso em que $A$ é unitária e de comprimento 4 existe $x \in A$ tal que $\left\{1_{A}, x, x^{2},\left(x^{2}\right)^{2}\right\}$ é base de $A$. A recíproca é imediata pois $l(A) \leq 4$ para qualquer álgebra unitária de dimensão 4 .

Exemplo 3.5. Considere a álgebra unitária $A$ com base $\left\{1_{A}, e_{1}, e_{2}, e_{3}\right\}$ e tabela de multiplicação dada por:

\begin{tabular}{|c|c|c|c|c|}
\hline$*$ & $1_{A}$ & $e_{1}$ & $e_{2}$ & $e_{3}$ \\
\hline $1_{A}$ & $1_{A}$ & $e_{1}$ & $e_{2}$ & $e_{3}$ \\
\hline$e_{1}$ & $e_{1}$ & $e_{2}$ & 0 & 0 \\
\hline$e_{2}$ & $e_{2}$ & 0 & $e_{3}$ & $e_{1}$ \\
\hline$e_{3}$ & $e_{3}$ & 0 & $e_{1}$ & $e_{1}$ \\
\hline
\end{tabular}

Fixando $S=\left\{e_{1}\right\}$ obtemos as igualdades $\mathcal{L}_{1}(S)=\left\langle 1_{A}, e_{1}\right\rangle, \mathcal{L}_{2}(S)=\mathcal{L}_{3}(S)=\left\langle 1_{A}, e_{1}, e_{2}\right\rangle, \mathcal{L}_{4}(S)=$ $\left\langle 1_{A}, e_{1}, e_{2}, e_{3}\right\rangle$, o que implica na igualdade $l(A)=4$.

Para as álgebras de comprimento 3 que não possuem identidade, existem elementos $x, y \in A$ tais que um dos conjuntos $\left\{x, y, x^{2}, x^{2} x\right\} ;\left\{x, y, x^{2}, x x^{2}\right\} ;\left\{x, y, x^{2}, x^{2} y\right\}\left\{x, y, x^{2}, y x^{2}\right\} ;\{x, y, x y, x(x y)\}$; $\{x, y, x y,(x y) x\} ;\{x, y, x y, y(x y)\}$ ou $\{x, y, x y,(x y) y\}$ é base de $A$, ou existe $x \in A$ tal que $\left\{x, x^{2}, x x^{2}, x^{2} x\right\}$ é base de $A$. A recíproca é falsa pois cada uma das condições da recíproca é satisfeita por pelo menos um dos conjuntos $S=\left\{e_{1}, e_{4}\right\}, T=\left\{e_{1}, e_{3}\right\}, U=\left\{e_{2}, e_{3}\right\}, V=\left\{e_{4}\right\}$ na álgebra do Exemplo 3.6. Supomos agora que $A$ é unitária e tem comprimento 3. Então existe um elemento $x \in A$ tal que $\left\{1_{A}, x, x^{2}, x x^{2}\right\}$ ou $\left\{1_{A}, x, x^{2}, x^{2} x\right\}$ é base de $A$. O conjunto $S=\left\{e_{2}\right\}$ no Exemplo 3.5 satisfaz as condições da recíproca, no entanto vale a igualdade $l(A)=4$.

Por fim, para as álgebras não unitárias de comprimento 2 temos que existem elementos $x, y, z \in A$ tais que um conjunto na forma $\left\{x, y, z, x^{2}\right\}$ ou $\{x, y, z, x y\}$ é uma base de $A$ ou elementos 
$x, y \in A$ tais que um conjunto na forma $\left\{x, y, x^{2}, y^{2}\right\} ;\left\{x, y, x^{2}, x y\right\} ;\left\{x, y, x^{2}, y x\right\}$ ou $\{x, y, x y, y x\}$ é uma base de $A$. A recíproca é falsa pois cada uma as condições da recíproca é satisfeita por pelo menos um dos conjuntos $S=\left\{e_{2}, e_{4}, e_{1}\right\}, T=\left\{e_{2}, e_{4}, e_{3}\right\}, V=\left\{e_{4}, e_{2}\right\}, W=\left\{e_{3}, e_{2}\right\}$ e $U=\left\{e_{4}, e_{1}\right\}$ na álgebra do Exemplo 3.6, no entanto vale a igualdade $l(A)=6$. Analogamente se a álgebra é unitária e de comprimento 2, existem elementos $x, y \in S$ tais que um dos conjuntos $\left\{1_{A}, x, y, x y\right\}$ ou $\left\{1_{A}, x, y, x^{2}\right\}$ é base de $A$. A recíproca é falsa como mostra o Exemplo 3.5 tomando os conjuntos $S=\left\{e_{2}, e_{3}\right\}$ e $T=\left\{e_{1}, e_{3}\right\}$, respectivamente.

Exemplo 3.6. Considere a álgebra com base $\left\{e_{1}, e_{2}, e_{3}, e_{4}\right\}$ e tabela de multiplicação dada por

\begin{tabular}{|c|c|c|c|c|}
\hline$*$ & $e_{1}$ & $e_{2}$ & $e_{3}$ & $e_{4}$ \\
\hline$e_{1}$ & $e_{2}$ & $e_{3}$ & 0 & $e_{2}$ \\
\hline$e_{2}$ & $e_{3}$ & $e_{3}$ & $e_{4}$ & $e_{1}$ \\
\hline$e_{3}$ & 0 & $e_{4}$ & $e_{1}$ & $e_{1}$ \\
\hline$e_{4}$ & $e_{3}$ & $e_{1}$ & $e_{1}$ & $e_{1}$ \\
\hline
\end{tabular}

Fixando $S=\left\{e_{1}\right\}$ temos que $\mathcal{L}_{1}(S)=\left\langle e_{1}\right\rangle, \mathcal{L}_{2}(S)=\left\langle e_{1}, e_{2}\right\rangle, \mathcal{L}_{3}(S)=\mathcal{L}_{4}(S)=\left\langle e_{1}, e_{2}, e_{3}\right\rangle, \mathcal{L}_{5}(S)=$ $\left\langle e_{1}, e_{2}, e_{3}, e_{4}\right\rangle$ e $\operatorname{assim} l(S)>4$. 


\section{Capítulo 4}

\section{O comprimento em Álgebras Associativas}

\subsection{A conjectura de Paz}

A análise das propriedades matriciais é um dos mais importantes ramos de pesquisa das álgebras associativas, além de presentes na modelagem de fenômenos na física, a utilização de matrizes para o estudo de estruturas como grupos e álgebras ganhou notoriedade através da teoria da representação.

O estudo do comportamento da dimensão de subálgebras comutativas nas álgebras de matrizes são datadas de 1905 nos trabalhos de Schur [23]. No entanto, instrumentos para avaliar o comportamento de uma álgebra associativa tornam-se cada vez mais escassos à medida que a dimensão se torna maior. Posto isso, é fundamental estabelecer uma relação entre dimensão e comprimento.

No Capítulo 2 foi estabelecido que uma álgebra não necessariamente associativa $A$ tem comprimento inferior a $2^{\operatorname{dim} A-1}$. Veremos nesta seção que esta cota superior na forma exponencial pode ser reduzida, assumindo associatividade, para a cota $\operatorname{dim} A-1$. Embora esse resultado represente uma melhora significativa, ao tratarmos do caso da álgebra das matrizes quadradas de ordem $n$ cujas entradas são elementos pertencentes a um corpo $K$, denotada por $M_{n}(K)$, temos um limitante na forma $l\left(M_{n}(K)\right) \leq n^{2}-1$. A tentativa de estabelecer uma cota superior para o comprimento de uma álgebra de matrizes na forma $l\left(M_{n}\right)=a n+b$ onde $a, b \in \mathbb{R}$, motivou Paz a estabelecer seguinte conjectura:

Conjectura 1 (Conjectura de Paz [20]). Sejam $K$ um corpo qualquer e $n$ um inteiro positivo. Então $l\left(M_{n}(K)\right) \leq 2 n-2$.

A validade da conjectura para os casos em que $n=2,3$ e 4 foi demonstrada por Paz em [20]. Adaptando o método utilizado por Pappacena [19], Shitov [24] demonstrou que para $n=5$ a conjectura também é verdadeira. O caso geral ainda segue em aberto, no entanto os trabalhos de Guterman e Markova [8] demonstram que, sob a hipótese do conjunto gerador possuir uma matriz não degenerada, a conjectura é verdadeira. 
No decorrer deste capítulo estudaremos, à luz dos teoremas vistos no Capítulo 3, como se comportam álgebras em dimensões inferiores a 6, refinando os resultados obtidos por meio do uso da associatividade.

\subsection{Resultados Complementares}

Relembramos nesta seção que uma álgebra $A$ é associativa quando vale a igualdade $(x y) z-$ $x(y z)=0$ para quaisquer elementos $x, y, z \in A$ e que consideraremos apenas álgebras de dimensão finita.

Vimos no Capítulo 2 que a presença de comutatividade não ocasiona alterações estruturais suficientes para que o limite $2^{\operatorname{dim} A-1}$ seja reduzido. Veremos, a seguir, como a associatividade afeta a estrutura dos espaços obtidos a partir de um conjunto gerador $S$, exemplificando, inicialmente, tal influência com o seguinte resultado.

Lema 4.1. [14] Sejam $A$ uma $K$-álgebra unitária e associativa e $S \subset A$ um conjunto gerador. Então $S_{i+j}=S_{i} \cdot S_{j} \cup S_{1}$ e, consequentemente $\mathcal{L}_{i+j}(S)=\left\langle\left(\mathcal{L}_{i}(S) \cdot \mathcal{L}_{j}(S)\right)+\mathcal{L}_{1}(S)\right\rangle$.

Demonstração. Suponha, sem perda de generalidade, que $i \leq j$. Segue imediatamente da aditividade do comprimento a inclusão $S_{i} \cdot S_{j} \cup S_{1} \subseteq S_{i+j}$. Reciprocamente, dado $x \in S_{i+j}$, se o comprimento de $x$ for 0 ou 1 então vale que $x \in S_{1}$; por outro lado, se for $l(x) \leq j$ então $x \in S_{j}$ enquanto se $i \leq l(x)=p \leq i+j$ escrevemos $x=x_{1} x_{2} \ldots x_{p}=\left(x_{1} \ldots x_{p-j}\right)\left(x_{p-j+1} \ldots x_{p}\right) \in S_{i} S_{j}$ o que demonstra a inclusão contrária. Tomando $x \in \mathcal{L}_{i+j}(S)$, escrevemos $x$ como combinação linear de palavras de comprimento menor que $p+1$, ou seja, $x=\left(\alpha_{p, 1} w_{p, 1}+\ldots+\alpha_{p, n_{p}} w_{p, n_{p}}\right)+\ldots+\left(\alpha_{1,1} w_{1,1}+\right.$ $\left.\ldots+\alpha_{1, n_{1}} w_{1, n_{1}}\right)+\alpha_{0} 1_{A}$, para alguns $\alpha_{i, j} \in K$ e com $w_{\lambda, r}$ denotando a $r$-ésima palavra de comprimento $\lambda$ presente na expressão de $x$. Como cada fator $\alpha_{t, m} w_{t, m}$ pertence ao espaço gerado por $S_{i} \cdot S_{j} \subset \mathcal{L}_{i}(S) \cdot \mathcal{L}_{j}(S)$, concluímos que $x$ é uma combinação linear de elementos de $\mathcal{L}_{i}(S) \cdot \mathcal{L}_{j}(S)$, assim $x \in\left\langle\mathcal{L}_{i}(S) \cdot \mathcal{L}_{j}(S)\right\rangle \subseteq\left\langle\left(\mathcal{L}_{i}(S) \cdot \mathcal{L}_{j}(S)\right)+\mathcal{L}_{1}(S)\right\rangle$. Para a inclusão contrária fixamos uma base $\left\{b_{1}, \ldots, b_{r}\right\}$ de $\left\langle\left(\mathcal{L}_{i}(S) \cdot \mathcal{L}_{j}(S)\right)+\mathcal{L}_{1}(S)\right\rangle$, escrevemos $b_{i}=\left(\sum_{k} \alpha_{k, i} z_{k}\right)\left(\sum_{t} \theta_{t, i} v_{t}\right)+\sum_{s} c_{s, i} u_{s}$, desse modo concluímos que $l\left(b_{i}\right) \leq i+j$, isto é, $b_{i} \in\left\langle S_{i} \cdot S_{j}\right\rangle=\mathcal{L}_{i+j}(S)$.

O Lema 4.1 mostra que as diferenças entre os elementos consecutivos da cadeia de um conjunto finito $S$ não podem ser arbitrariamente pequenas sem que isso remova a possibilidade de que $S$ seja um conjunto gerador. Veremos no lema a seguir que se dois elementos consecutivos da cadeia de um subconjunto finito $S$ coincidem, então $S$ não pode ser um conjunto gerador.

Lema 4.2. [7] Sejam $A$ uma $K$-álgebra associativa e $S$ um subconjunto finito de $A$. $\operatorname{Se} \operatorname{dim} \mathcal{L}_{n}(S)=$ $\operatorname{dim} \mathcal{L}_{n+1}(S)$, então $\operatorname{dim} \mathcal{L}_{n}(S)=\operatorname{dim} \mathcal{L}_{h}(S)$ para todo $h \geq n$.

Demonstração. Pelo Lema 4.1 temos $\mathcal{L}_{n+2}(S)=\left\langle\left(\mathcal{L}_{1}(S) \cdot \mathcal{L}_{n+1}(S)\right)+\mathcal{L}_{1}(S)\right\rangle=\left\langle\left(\mathcal{L}_{1}(S) \cdot \mathcal{L}_{n}(S)\right)+\right.$ $\left.\mathcal{L}_{1}(S)\right\rangle=\mathcal{L}_{n+1}(S)$. Prosseguimos por indução no acréscimo $k$ ao índice $n$ em $\operatorname{dim} \mathcal{L}_{n}(S)$ e concluí$\operatorname{mos}$ que $\operatorname{dim} \mathcal{L}_{n}(S)=\operatorname{dim} \mathcal{L}_{n+k}(S)$.

Podemos utilizar o Lema 4.1 para demonstrar o seguinte teorema.

Teorema 4.1. [19] Seja A uma K-álgebra associativa com $\operatorname{dim} A=n$, então $l(A) \leq n-1$ se A for unitária el $(A) \leq n$ caso contrário. 
Demonstração. Pelo Lema 4.2, se $a_{0} \rightarrow a_{1} \rightarrow \cdots \rightarrow a_{k}=n$ é uma cadeia de um conjunto gerador $S$ de $A$, então $a_{l}<a_{l+1}$, para todo $l$ tal que $l+1 \leq k$, isto é, cada termo da cadeia recebe o acréscimo de no mínimo uma unidade em relação ao termo anterior. Portanto, a cadeia de $S$ terá no máximo $n-a_{0}$ termos, ou seja, $l(S) \leq n-a_{0}$. Logo, $l(A) \leq n-1$ se $A$ for unitária e $l(A) \leq n$, caso contrário.

Os próximos resultados são modificações de algumas das conclusões encontradas no trabalho de Guterman e Markova em [9], onde eles estudaram as propriedades do comprimento em álgebras de matrizes e derivaram diversas relações.

Corolário 4.1. [13] Seja $A$ uma $K$-álgebra associativa unitária e de dimensão $n$. Então $l(A)=n-1$ se, e somente se, $A$ é comutativa e existe um elemento $v \in A$ tal que $A$ é a álgebra gerada por $v$.

Demonstração. Vamos mostrar que se $l(A)>n-2$ então todo conjunto gerador $S$ possui apenas um elemento. De fato, observe que $n>a_{n-2}=a_{1}+\sum_{i=2}^{n-2}\left(a_{i}-a_{i-1}\right) \geq a_{1}+(n-3)=|S|+1+(n-3)$, o que implica $2>|S|>0$. Desse modo, existe um elemento $v \in A$ tal que $S=\{v\}$ e a álgebra gerada pelo vetor $v$ é $A$. Assim, como $A$ é associativa, também é de potências associativas e então as potências de $v$ comutam, ou seja, a álgebra gerada por $v$ é comutativa. A recíproca é imediata.

Corolário 4.2. [13] Se $A$ é uma $K$-álgebra associativa de dimensão $n$ que não é unitária, então $l(A)=n$ se, e somente se, $A$ é comutativa e existe um elemento $v \in A$ tal que $A$ é a álgebra gerada por $v$.

O Teorema 4.1 pode ser utilizado para demonstrar um caso semelhante ao enunciado na conjectura de Paz, obtido se considerarmos que a álgebra em questão pode ser gerada por um par de matrizes que comutam. Este resultado será o conteúdo do Corolário 4.3, no entanto antes de demonstrá-lo, precisaremos do seguinte teorema auxiliar.

Teorema 4.2. [5] Se duas matrizes $P, Q \in M_{n}(K)$ comutam, então a subálgebra de $M_{n}(K)$ gerada por $P$ e $Q$ tem dimensão no máximo $n$.

Pelo Teorema 4.1 o comprimento da álgebra $A$ considerada no teorema acima não excede o valor $\operatorname{dim} A$ - 1 . Complementando o resultado obtido com a relação $\operatorname{dim} A \leq n$ fornecida pelo Teorema 4.2 concluímos que o comprimento de $A$ não excede o valor $n-1$, demonstrando o corolário a seguir.

Corolário 4.3. [9] Sejam $P$ e $Q$ duas matrizes pertencentes a $M_{n}(K)$ que comutam. Então a subálgebra $\mathcal{A} \subset A$ gerada por $P$ e $Q$ satisfaz $l(\mathcal{A}) \leq n-1$.

O Corolário 4.3 demonstra que a conjectura de Paz é verdadeira se considerada para subálgebras de $M_{n}(K)$ que são geradas por dois elementos que comutam. Mais geralmente, se uma subálgebra comutativa está contida em uma álgebra associativa, o seguinte resultado é verdadeiro.

Teorema 4.3. [9] Sejam K um corpo arbitrário e A uma subálgebra comutativa de $M_{n}(K)$. Então $l\left(M_{n}(K)\right) \leq$ $n-1$.

Corolário 4.4. [9] Seja $D_{n}(K)$ a álgebra das matrizes quadradas e diagonais de ordem $n$ sobre um corpo $K$. Então $l\left(D_{n}(K)\right) \leq n-1$. Além disso, se $|K| \geq n$ a cota é atingida. 
Demonstração. Pelo Teorema 4.1 concluímos que $l\left(D_{n}(K)\right) \leq n-1$. Sejam $a_{1}, \ldots, a_{n}$ elementos distintos de $K$ e considere a matriz $D=\left\{d_{i j}\right\}$ satisfazendo $d_{i j}=0$ se $i \neq j$ e $\left\{d_{i i}=a_{i}\right\}$, isto é, $D=\operatorname{diag}\left\{a_{1}, \ldots, a_{n}\right\}$. Note que como o polinômio minimal e o polinômio característico de $D$ coincidem, se o conjunto $\left\{I, D, \ldots, D^{n-1}\right\}$ fosse linearmente dependente existiria um polinômio $p(T)$ de grau menor que $n$ que anula $D$, o que contraria a minimalidade do polinômio minimal de $D$. Portanto $D$ satisfaz a igualdade $l(D)=n-1$.

Lema 4.3. [14] Sejam $A$ e $B$ duas $K$-álgebras associativas e $f: A \rightarrow B$ um homomorfismo sobrejetivo. Então $l(B) \leq l(A)$.

Demonstração. Pelo teorema fundamental dos homomorfismos de álgebras sabemos que $B \simeq A / \operatorname{Ker} f$. Fixe um conjunto gerador $S_{B}=\left\{b_{1}, \ldots, b_{m}\right\}$ de $B$ com comprimento $l(B)=l\left(S_{B}\right)$. Sejam $a_{1}, \ldots, a_{m}$ elementos de $A$ tais que $f\left(a_{j}\right)=b_{j}, j=1, \ldots, m$, e $\left\{e_{1}, \ldots, e_{k}\right\}$ uma base $\operatorname{de} \operatorname{Ker} f$. Temos que $S_{A}=\left\{a_{1}, \ldots, a_{m}, e_{1}, \ldots, e_{k}\right\}$ é um conjunto gerador de $A$.

Seja $n=l\left(S_{A}\right)$. Afirmamos que $l\left(S_{B}\right) \leq n$. Para provar a afirmação, vamos mostrar que $w \in \mathcal{L}_{n}\left(S_{B}\right)$ para qualquer elemento $w \in \mathcal{L}_{n+1}\left(S_{B}\right)$. Pela definição de $w$, existe um polinômio $P_{n+1} \in K\left[x_{1}, \ldots, x_{m}\right]$ de grau menor que $n+2$ tal que $w=P_{n+1}\left(b_{1}, \ldots, b_{m}\right)$. Então $f(v)=w$, onde $v=P_{n+1}\left(a_{1}, \ldots, a_{m}\right)$. Por construção, $v \in \mathcal{L}_{n+1}\left(S_{A}\right)$. Consequentemente, temos que $v \in$ $\mathcal{L}_{n}\left(S_{A}\right)$, isto é, existe um polinômio $P_{n} \in K\left[x_{1}, \ldots, x_{m+k}\right]$ de grau menor que ou igual a $n$ tal que $v=P_{n}\left(a_{1}, \ldots, a_{m}, e_{1}, \ldots, e_{k}\right)$. Como Ker $f$ é um ideal de $A$, temos que existe um polinômio $Q_{n} \in K\left[x_{1}, \ldots, x_{m}\right]$ tal que $v=Q_{n}\left(a_{1}, \ldots, a_{m}\right)+r \operatorname{com} r \in K \subseteq \mathcal{L}_{1}\left(S_{A}\right)$. Desse modo, segue que

$$
w=f(v)=f\left(Q_{n}\left(a_{1}, \ldots, a_{m}\right)+r\right)=f\left(Q_{n}\left(a_{1}, \ldots, a_{m}\right)\right)=Q_{n}\left(b_{1}, \ldots, b_{m}\right) \in \mathcal{L}_{n}\left(S_{B}\right) .
$$

Consequentemente, $l(B)=l\left(S_{B}\right) \leq l\left(S_{A}\right) \leq l(A)$.

Quando o homomorfismo $f: A \rightarrow B$ é bijetivo, podemos aplicar o lema anterior aos homomorfismos $f$ e $f^{-1}$ para demonstrar a igualdade $l(A)=l(B)$.

Uma questão levantada naturalmente é se a existência de um homomorfismo injetivo $f: A \rightarrow$ $B$ implica na relação $l(A) \leq l(B)$. A resposta para essa pergunta é negativa. Vale ainda que para todo inteiro positivo $i$, existe um inteiro $n$ e $K$-álgebras $\mathcal{A}^{\prime} \subset \mathcal{A} \subset M_{n}(K)$ satisfazendo $l\left(\mathcal{A}^{\prime}\right)-l(\mathcal{A})=i$, esta construção pode ser consultada em [17].

A igualdade entre comprimentos não é uma garantia de isomorfismo pois, por exemplo, duas zero álgebras de dimensões distintas e sobre o mesmo corpo possuem o mesmo comprimento, mas não são isomorfas.

\section{3 Álgebras associativas de dimensões menores ou iguais a 4}

Quando $A$ é uma álgebra de dimensão 5, podemos utilizar o Teorema 4.1 para concluir que $l(A) \leq$ 4 se $A$ é unitária e $l(A) \leq 5$ caso contrário. Por outro lado, quando $A$ é uma álgebra associativa de dimensão 4, podemos utilizar a teoria desenvolvida no Capítulo 3 para obter os seguintes resultados. 
Teorema 4.4. Se A é uma K-álgebra associativa de dimensão 4, então são verdadeiras as seguintes afirmações:

- A tem comprimento 4 se, e somente se, não é unitária e possui um elemento $x$ tal que o conjunto $\left\{x, x^{2}, x^{3}, x^{4}\right\}$ é base de $A$;

- Se A possui identidade, então A tem comprimento 3 se, e somente se, possui um elemento $x$ tal que o conjunto $\left\{1, x, x^{2}, x^{3}\right\}$ é base de $A$;

- Se A não possui identidade e tem comprimento 2, então existem elementos $x, y, z \in A$ tais que $\{x, y, z, w\}$ é uma base com $w \in\left\{x^{2}, x y, x z, y z\right\}$ ou existem elementos $x, y \in A$ tais que $\left\{x, y, w_{1}, w_{2}\right\}$ é base de $A$, onde $w_{1}, w_{2} \in\left\{x^{2}, y^{2}, x y, y x\right\}$;

- Se A possui identidade e tem comprimento 2, então possui elementos x e y tais que um dos conjuntos $\{1, x, y, x y\}$ ou $\left\{1, x, y, x^{2}\right\}$ é base de $A$;

- A possui identidade e tem comprimento 1 se, e somente se, para quaisquer $x, y \in A$, vale a relação $x y \in\langle 1, x, y\rangle$;

- A não é unitária e tem comprimento 1 se, e somente se, para quaisquer $x, y \in A$ vale a relação $x y \in\langle x, y\rangle$.

A próxima etapa desse capítulo pode ser resumida no estudo do comportamento do comprimento em uma álgebra associativa de dimensão inferior a 4 . Fazemos menção que o caso $\operatorname{dim} A=1$ já foi estudado no Capítulo 3. Para o caso em que $\operatorname{dim} A=2$ o Teorema 4.1 fornece a relação $l(A) \leq 1$ e a igualdade está estabelecida nos Corolários 4.1 e 4.2, enquanto para o caso $\operatorname{dim} A=3$ vale que $l(A) \leq 3$. Revisamos o Teorema 3.2 utilizando os resultados desta seção e condensamos as conclusões no teorema a seguir.

Teorema 4.5. Se A é uma K-álgebra associativa de dimensão 3, então são verdadeiras as seguintes afirmações:

- A tem comprimento 3 se, e somente se, não possui identidade e existe um elemento $x$ tal que $\left\{x, x^{2}, x^{3}\right\}$ ébase de A;

- Se A tem comprimento 2 e não possui identidade, então existem elementos $x, y \in A$ tais que um dos conjuntos $\{x, y, x y\},\left\{x, y, x^{2}\right\}$ é base de $A$;

- Se A é unitária, então tem comprimento 2 se, e somente se, possui um elemento $x$ tal que $\left\{1, x, x^{2}\right\}$ é base de A;

- A possui identidade e tem comprimento 1 se, e somente se, para quaisquer $x, y \in A$, vale a relação $x y \in\langle 1, x, y\rangle$;

- A não é unitária e tem comprimento 1 se, e somente se, para quaisquer $x, y \in A$ vale a relação $x y \in\langle x, y\rangle$.

Embora os teoremas desta seção não ofereçam uma classificação das álgebras associativas de dimensão menor que 5 a menos de isomorfismo, oferecem a possibilidade de reduzir o problema de determinar o comprimento à busca de vetores específicos, o que por vezes representa uma saída computacional mais simples para o problema. 


\section{Capítulo 5}

\section{O comprimento em Álgebras Alternativas}

\subsection{Introdução}

Neste capítulo iremos calcular o comprimento das álgebras alternativas que não são associativas e que possuem dimensão inferior a 6, sob hipóteses adequadas, dividiremos as álgebras alternativas em classes de isomorfismos e então determinaremos o comprimento de cada classe. Além disso, mostraremos que as álgebras alternativas de dimensão inferior a 4 são associativas. Para isto, começaremos estabelecendo limites superiores para tais comprimentos, limitantes estes que seguem de propriedades tais como as identidades de Moufang e o Teorema de Artin.

Estabelecidos os limitantes, iremos construir outras ferramentas que auxiliam na determinação das álgebras que possuem comprimento 3 e na fabricação de conjuntos geradores de comprimento 3, validando assim a afirmação. Por fim, ao final da seção determinaremos os componentes de cada uma das classes de equivalência, isto é, munidos do fato de que uma álgebra alternativa de dimensão menor que 6 tem comprimento 2, esta é isomorfa a uma das álgebras mencionadas, ademais, se uma álgebra alternativa é isomorfa a uma das álgebras expostas, seremos capazes de determinar seu comprimento.

\subsection{Resultados preliminares}

Relembramos que durante esta seção faremos uso constante das propriedades das palavras irredutíveis, por vezes de forma implícita.

Lema 5.1 (Identidades de Moufang). Em uma álgebra alternativa $A$, são válidas as seguintes identidades:

$$
\begin{array}{ll}
(x a x) y=x(a(x y)) & \text { (Identidade de Moufang à esquerda) } \\
y(x a x)=((y x) a) x & \text { (Identidade de Moufang à direita) } \\
(x y)(a x)=x(y a) x & \text { (Identidade de Moufang ao meio) }
\end{array}
$$


Para a primeira igualdade observe que: $(x a x) y-x(a(x y))=(x a, x, y)+(x, a, x y)=-(x, x a, y)-$ $(x, x y, a)=-(x(x a)) y+x((x a) y)-(x(x y)) a+x((x y) a)=-\left(x^{2} a\right) y-\left(x^{2} y\right) a+x((x a) y+(x y) a)=$ $-\left(x^{2}, a, y\right)-\left(x^{2}, y, a\right)-x^{2}(a y)-x^{2}(y a)+x((x a) y+(x y) a)=x(-x(a y)-x(y a)+(x a) y+(x y) a)=$ $x((x, a, y)+(x, y, a))=0$.

Provamos a segunda de forma análoga utilizando a álgebra oposta. Para o que falta, note que a primeira igualdade nos dá que: $(x y)(a x)-x(y a) x=(x, y, a x)+x[y(a x)-(y a) x]=-(x, a x, y)-$ $x(y, a, x)=-(x a x) y+x[(a x) y-(y, a, x)]=-x[a(x y)-(a x) y+(y, a, x)]=-x[-(a, x, y)+(y, a, x)]=0$.

Teorema 5.1. (Teorema de Artin) Se A é uma K-álgebra alternativa então toda subálgebra gerada por dois elementos de A é associativa.

Demonstração. Se $x$ e y são quaisquer dois elementos de $A$, denote por $p=p(x, y)$ qualquer produto não associativo $z_{1} z_{2} \ldots z_{t}$ (com alguma distribuição de parênteses) de $t$ fatores $z_{i} \in\{x, y\}$. Denote ainda por $l(p)$ o comprimento $t$ de um tal produto. É suficiente provar que vale $(p, q, r)=0$ para todos produtos não associativos $p=p(x, y), q=q(x, y)$ e $r=r(x, y)$.

A demonstração será por indução em $n=l(p)+l(q)+l(r)$. Note que para $n<3$, pelo menos um dos produtos não associativos $p, q, r$ é nulo e o resultado é imediato. Assim, vamos supor $l(p) \geq 1, l(q) \geq 1$ e $l(r) \geq 1$. Suponha, por hipótese de indução, que vale $(p, q, r)=0$ se $l(p)+l(q)+l(r)<n$. Como $l(p)<l(p)+l(q)+l(r)=n$, podemos usar a hipótese de indução para elementos do produto $p$, ou seja, quaisquer 3 produtos de elementos de $p$ são associativos e, consequentemente, pela lei associativa generalizada, não é necessário colocar parênteses no produto $p=p(x, y)=z_{1} z_{2} \ldots z_{t}$ e podemos dizer que $p$ começa em $z_{1}$.

Sabemos que $p=p(x, y), q=q(x, y)$ e $r=r(x, y)$, assim, obrigatoriamente, dois dos produtos $p, q, r$ devem começar com a mesma letra, digamos $x$. Como associadores alternam em $A$, podemos assumir que $q$ e $r$ começam com $x$ pois se fossem $p$ e $r$, bastaria escrever $(p, q, r)=(r, p, q)$; se fossem $p$ e $r$, bastaria escrever $(p, q, r)=-(q, p, r)$. Se $l(q)>1$ e $l(r)>1$, então $q=x q^{\prime}$ e $r=x r^{\prime}$, onde $l\left(q^{\prime}\right)=l(q)-1$ e $l\left(r^{\prime}\right)=l(r)-1$. Agora $(p, q, r)=\left(p, x q^{\prime}, x r^{\prime}\right)=-\left(x r^{\prime}, x q^{\prime}, p\right)$.

Observe que $(y, x a, x)=(y(x a)) x-y(x a x)$ e pela identidade de Moufang à direita concluímos que $(y, x a, x)=-(y, x, a) x$. Linearizando esta identidade, obtemos

$$
(y, x a, z)+(y, z a, x)=-(y, x, a) z-(y, z, a) x
$$

Substituindo $y=x r^{\prime}, a=q^{\prime}$ e $z=p$, temos $-\left(x r^{\prime}, x q^{\prime}, p\right)=\left(x r^{\prime}, p q^{\prime}, x\right)+\left(x r^{\prime}, x, q^{\prime}\right) p+\left(x r^{\prime}, p, q^{\prime}\right) x=$ $\left(x r^{\prime}, p q^{\prime}, x\right)$, onde na primeira igualdade utilizamos a igualdade (5.1) e na segunda igualdade utilizamos a hipótese de indução. Em cada associador, temos

- $\left(x r^{\prime}, p q^{\prime}, x\right) \rightarrow l\left(x r^{\prime}\right)+l\left(p q^{\prime}\right)+l(x)=1+l\left(r^{\prime}\right)+l(p)+l\left(q^{\prime}\right)+1=1+(-1+l(r))+l(p)+(-1+$ $l(q))+1=l(p)+l(q)+l(r)=n$;

- $\left(x r^{\prime}, x, q^{\prime}\right) \rightarrow l\left(x r^{\prime}\right)+l(x)+l\left(q^{\prime}\right)=1+l\left(r^{\prime}\right)+1+l\left(q^{\prime}\right)=1+(-1+l(r)+1+(-1+l(q))=l(q)+$ $l(r)<l(p)+l(q)+l(r)=n$

- $\left(x r^{\prime}, p, q^{\prime}\right) \rightarrow l\left(x r^{\prime}\right)+l(p)+l\left(q^{\prime}\right)=1+(-1+l(r))+(-1+l(q))+l(p)=l(p)+l(q)+l(r)-1<$ $l(p)+l(q)+l(r)=n$. 
Observe agora que $\left(x r^{\prime}, p q^{\prime}, x\right)=-\left(p q^{\prime}, x r^{\prime}, x\right)=\left(p q^{\prime}, x, r^{\prime}\right) x=0$, pois $l(p)+l\left(q^{\prime}\right)+\nmid(x)+l\left(r^{\prime}\right)=$ $l(p)-1+l(q)+1-1+l(r)<n$. Se apenas um dos produtos $q, r$ tem grau maior que 1 , digamos $q=x q^{\prime}$, então temos $(p, q, r)=\left(p, x q^{\prime}, r\right)=-\left(p, x, q^{\prime}\right) x=0$, pois $l(p)+l(x)+l\left(q^{\prime}\right)=l(p)+1-1+$ $l(q)=l(p)+l(q)<n$. Finalmente, o caso em que $l(q)=l(r)=1$ é dado pela identidade alternativa à direita $(p, q, r)=(p, x, x)=0$.

Corolário 5.1. Uma $K$-álgebra alternativa é de potências associativas.

Lema 5.2. [6] Seja $A$ uma $K$-álgebra alternativa com um idempotente $e \neq 0$ e decomposição de Peirce dada por $A=A_{11} \oplus A_{10} \oplus A_{01} \oplus A_{00}$. Se $A_{11}$ e $A_{00}$ são associativas e $A_{10} A_{10}=A_{01} A_{01}=0$, então $A$ é associativa.

Demonstração. Sejam $A_{i j}, A_{k l}$ e $A_{m n}$ componentes de Peirce de $A$. Precisamos provar que $\left(A_{i j}, A_{k l}, A_{m n}\right)=$ 0 para quaisquer $i, j, k, l, m, n \in\{0,1\}$. Vamos dividir a demonstração em 3 casos.

Caso 1: As três componentes de um associador são iguais.

Neste caso, decorre das hipóteses que $\left(A_{11}, A_{11}, A_{11}\right)=0$ e $\left(A_{00}, A_{00}, A_{00}\right)=0$, pois $A_{11}$ e $A_{00}$ são associativas e $\left(A_{10}, A_{10}, A_{10}\right)=0$ e $\left(A_{01}, A_{01}, A_{01}\right)=0$, pois $A_{10} A_{10}=0$ e $A_{01} A_{01}=0$.

Caso 2: As três componentes de um associador são diferentes.

Permutando a ordem dos argumentos, é suficiente considerar as $\left(A_{11}, A_{10}, A_{01}\right),\left(A_{11}, A_{10}, A_{00}\right)$, $\left(A_{11}, A_{01}, A_{00}\right)$ e $\left(A_{10}, A_{01}, A_{00}\right)$. Observe que

- $\left(A_{11}, A_{10}, A_{01}\right)=-\left(A_{10}, A_{11}, A_{01}\right) \subseteq 0 \cdot A_{01}+A_{10} \cdot 0=0$;

- $\left(A_{11}, A_{10}, A_{00}\right)=-\left(A_{10}, A_{11}, A_{00}\right) \subseteq 0 \cdot A_{00}+A_{10} \cdot 0=0$;

- $\left(A_{11}, A_{01}, A_{00}\right)=0$, pois $A_{11} A_{01}=A_{01} A_{00}=0$;

- $\left(A_{10}, A_{01}, A_{00}\right) \subseteq A_{11} A_{00}-A_{10} \cdot 0=0$.

Caso 3: Exatamente duas componentes de um associador são diferentes.

Em vista de que $A$ é uma álgebra alternativa, basta analisar os seguintes 12 associadores:

- $\left(A_{11}, A_{11}, A_{10}\right)=-\left(A_{10}, A_{11}, A_{11}\right) \subseteq 0 \cdot A_{11}+A_{10} A_{11}=0$;

- $\left(A_{11}, A_{11}, A_{01}\right)=0$, pois $A_{11} A_{01}=0$;

- $\left(A_{11}, A_{11}, A_{00}\right)$, pois $A_{11} A_{00}=0$;

- $\left(A_{10}, A_{10}, A_{11}\right)$, pois $A_{10} A_{10}=0$ e $A_{10} A_{11}=0$;

- $\left(A_{10}, A_{10}, A_{01}\right)=0$, pois $A_{10} A_{10}=0$ e $A_{10} A_{11}=0$;

- $\left(A_{10}, A_{10}, A_{00}\right)=0$, pois $A_{10} A_{10}=0$;

- $\left(A_{01}, A_{01}, A_{11}\right)=0$, pois $A_{01} A_{01}=0$;

- $\left(A_{01}, A_{01}, A_{10}\right) \subseteq 0 \cdot A_{10}+A_{01} A_{00}=0$;

- $\left(A_{01}, A_{01}, A_{00}\right) \subseteq 0 \cdot A_{00}+A_{01} \cdot 0=0$;

- $\left(A_{00}, A_{00}, A_{11}\right)=0$, pois $A_{00} A_{11}=0$;

- $\left(A_{00}, A_{00}, A_{10}\right)=0$, pois $A_{00} A_{10}=0$; 
- $\left(A_{00}, A_{00}, A_{01}\right)=-\left(A_{01}, A_{00}, A_{00}\right)=0$.

O que conclui a demonstração.

Por fim, encerramos a seção com dois teoremas estruturais sobre álgebras alternativas.

Teorema 5.2. [Wedderburn-Artin [15], p.138] Sejam A uma K-álgebra associativa com dimensão finita e $J(A)$ seu radical de Jacobson. Então existem inteiros positivos $n_{1}, \ldots, n_{r}$ e álgebras com divisão $D_{1}, \ldots, D_{r}$ tais que $A / J(A) \simeq M_{n_{1}}\left(D_{1}\right) \oplus \cdots \oplus M_{n_{r}}\left(D_{r}\right)$, onde $M_{n_{s}}\left(D_{s}\right)$ denota o anel de matrizes de ordem $n_{s}$ com entradas em $D_{s}$. Além disso, se $A / J(A) \simeq M_{t_{1}}\left(D_{1}^{\prime}\right) \oplus \cdots \oplus M_{t_{s}}\left(D_{s}^{\prime}\right)$ é outra decomposição temos $r=s$, $t_{i}=n_{i}$ e $D_{i}^{\prime} \simeq D_{i}$, para $i=1, \ldots$, s reordenando os índices, se necessário.

Teorema 5.3 (Wedderburn [22]). Seja A uma K-álgebra alternativa de dimensão finita com radical $R(A)$. Se $A / R(A)$ é separável, então $A=R(A) \oplus A / R(A)$.

\subsection{Classificação das álgebras de dimensão menor que 6}

Iniciaremos esta seção com a classificação das álgebras alternativas que não são associativas e de dimensão menor que 6 sobre um corpo perfeito, e utilizaremos este resultado para determinar os possíveis comprimentos de uma álgebra alternativa de dimensão menor que 6 sobre um corpo perfeito.

Teorema 5.4. Seja K um corpo arbitrário. Toda K-álgebra alternativa de dimensão 3 é associativa.

Demonstração. Se $A$ é unitária então é uma álgebra gerada por três elementos que associam, portanto é associativa. Podemos supor então que a álgebra não possui elemento identidade. Dividiremos a demonstração em dois casos: $A$ é nilálgebra e $A$ não é nilálgebra.

Lema 5.3. Se $A$ é uma nilálgebra alternativa de dimensão 3 sobre um corpo $K$, então $A$ é associativa.

Demonstração. Suponha que exista uma nilálgebra alternativa $A$ de dimensão 3 que não é associativa. Pelo Teorema de Artin temos que $l(A)=1$ e pela Proposição 3.1 segue que $x y \in\langle x, y\rangle$ para quaisquer $x, y \in A$. Fixamos uma base $\{a, b, c\}$ para a álgebra $A$. Seja $n$ o menor inteiro positivo satisfazendo $a^{n}=0$, pela Proposição 3.1 temos que $a^{2}=p a$ com $p \in K$, e então $0=a^{n}=p^{n-1} a$ o que implica em $p=0$. Em particular, $a^{2}=b^{2}=c^{2}=0$.

Novamente pela Proposição 3.1 temos que $a b=t_{1} a+t_{2} b$ com $t_{1}, t_{2} \in K$. Segue da identidade $a^{2} b=a(a b)$ que $0=a\left(t_{1} a+t_{2} b\right)=t_{2} a b=t_{1} t_{2} a+t_{2}^{2} b$. Como $a, b$ são linearmente independentes, devemos ter $t_{2}=0$ e então $a b=t_{1} a$. De modo análogo obtemos as igualdades $a c=k_{1} a, b c=\lambda_{1} b$ e $b a=r_{1} b$, onde $r_{1}, \lambda_{1}, k_{1} \in K$. Novamente temos que $(a b)(a b) \in\langle a b\rangle$ e então podemos escrever $(a b)^{2}=p_{1} a b$ para algum $p_{1} \in K$. Segue dessas observações que $0=a^{2}=0=t_{1}^{2} a^{2}=\left(t_{1} a\right)^{2}=(a b)^{2}=$ $p_{1} a b$, ou seja, $a b=0$ ou $p_{1}=0$.

Se $a b=0$, então $(a, b, c)=(a b) c-a(b c)=-a\left(\lambda_{1} b\right)=-\lambda_{1} a b=0$ e então $A$ seria associativa. Assumindo então que $p_{1}=0$ temos que $(a b)^{2}=0$ e, pelo Teorema de Artin, $0=(a b)(a b)=$ $\left.a(b(a b))=a\left(b\left(t_{1} a\right)\right)=a\left(t_{1} r_{1} b\right)\right)=t_{1} r_{1}(a b)$. Como $a b=0$ viola a não associatividade de $A$, devemos ter $t_{1} r_{1}=0$, isto é, $r_{1}=0$ pois $t_{1}=0$ implica em $a b=0$. Se $r_{1}=0$ então $b a=0$. Assim, 
$(b, a, c)=(b a) c-b(a c)=-b(a c)=-b\left(k_{1} a\right)=-k_{1} b a=0$ e novamente $A$ seria associativa, absurdo.

Para o caso em que $A$ não é uma nilálgebra temos o seguinte resultado.

Lema 5.4. Se $A$ é uma $K$-álgebra alternativa de dimensão 3 que não é uma nilálgebra, então $A$ é associativa.

Demonstração. Suponha que exista uma álgebra $A$ com dimensão 3 que é alternativa mas que não é associativa. Pelo que foi feito, sabemos que $A$ não pode ser unitária, tem comprimento 1 e seus elementos satisfazem a relação $x y \in\langle x, y\rangle$ para quaisquer $x, y \in A$. Além disso, pelo Lema 5.3 sabemos que $A$ não é uma nilálgebra.

Como $A$ é uma álgebra alternativa que não é uma nilágebra e também não possui elemento identidade, concluímos que $A$ possui um idempotente não trivial $e$. Consideramos a decomposição de Peirce em relação ao idempotente $e, A=A_{11} \oplus A_{10} \oplus A_{01} \oplus A_{00}$. Se $\operatorname{dim} A_{11}=3$, então $A=A_{11}$, consequentemente $e$ seria o elemento identidade, um absurdo.

Suponha então que $\operatorname{dim} A_{11}=2$. Se $\operatorname{dim} A_{00}=1$ então $A_{11}, A_{00}$ são associativas pois $A_{00}$ é uma subálgebra de dimensão 1 , vale ainda que $A_{10} A_{10}=A_{01} A_{01}=0$ e pelo Lema 5.2, $A$ seria associativa. Se $\operatorname{dim} A_{11}=2$ e $\operatorname{dim} A_{00}=0$ então pelo menos um dos fatores $A_{01}$ e $A_{10}$ tem dimensão zero. Podemos supor sem perda de generalidade que $\operatorname{dim} A_{10}=0$. Segue de $A_{01} A_{01} \subseteq A_{10}=0$ que $A_{11}, A_{00}$ são associativas e $A_{01} A_{01}=0=A_{10} A_{10}$ e novamente pelo Lema 5.2, $A$ seria associativa, absurdo.

A discussão acima mostra que $\operatorname{dim} A_{11}=\operatorname{dim} A_{10}=\operatorname{dim} A_{01}=1$. Se $0 \neq A_{10} A_{01} \subseteq A_{11}$, existem elementos não nulos $c \in A_{10}$ e $b \in A_{01}$ tais que $0 \neq b c=a \in A_{11}$, contrariando o fato de que $c b \in\langle b, c\rangle$. Assim, devemos ter que $A_{10} A_{01}=0$ e $A_{01} A_{10} \subseteq A_{00}=0$. Além disso, o conjunto $\{e, c, b\}$ é uma base de $A$.

Como $A$ é alternativa, é suficiente mostrar que o associador $(e, b, c)$ é nulo. Note que $(e b) c=$ 0 e que $b c \in A_{00}=\{0\}$ portanto $e(b c)=0$. Isto mostra que $(e, b, c)=0$ e então $A$ é associativa.

Os Lemas 5.3 e 5.4 mostram que uma álgebra que não possui elemento identidade é associativa. Como uma álgebra de dimensão 3 que é unitária e alternativa é associativa, o resultado está provado.

Teorema 5.5. [6] Seja A uma K-álgebra alternativa, mas que não é associativa, de dimensão menor que 6 sobre um corpo perfeito, então A é isomorfa a uma das seguintes álgebras:

\begin{tabular}{|c|c|c|c|c|}
\hline 4.1 & $e$ & $a$ & $b$ & $c$ \\
\hline$e$ & $e$ & 0 & $b$ & $c$ \\
\hline$a$ & $a$ & 0 & 0 & 0 \\
\hline$b$ & 0 & 0 & 0 & $a$ \\
\hline$c$ & 0 & 0 & $-a$ & 0 \\
\hline
\end{tabular}

\begin{tabular}{|c|c|c|c|c|}
\hline $4.1^{\text {op }}$ & $e$ & $a$ & $b$ & $c$ \\
\hline$e$ & $e$ & $a$ & 0 & 0 \\
\hline$a$ & 0 & 0 & 0 & 0 \\
\hline$b$ & $b$ & 0 & 0 & $-a$ \\
\hline$c$ & $c$ & 0 & $a$ & 0 \\
\hline
\end{tabular}




\begin{tabular}{|l|l|l|l|l|l|}
\hline 5.1 & $e$ & $a$ & $b$ & $c$ & $d$ \\
\hline$e$ & $e$ & 0 & $b$ & $c$ & $d$ \\
\hline$a$ & $a$ & 0 & 0 & 0 & 0 \\
\hline$b$ & 0 & 0 & 0 & $a$ & 0 \\
\hline$c$ & 0 & 0 & $-a$ & 0 & 0 \\
\hline$d$ & $d$ & 0 & 0 & 0 & 0 \\
\hline
\end{tabular}

\begin{tabular}{|c|c|c|c|c|c|}
\hline $5.1^{o p}$ & $e$ & $a$ & $b$ & $c$ & $d$ \\
\hline$e$ & $e$ & $a$ & 0 & 0 & $d$ \\
\hline$a$ & 0 & 0 & 0 & 0 & 0 \\
\hline$b$ & $b$ & 0 & 0 & $-a$ & 0 \\
\hline$c$ & $c$ & 0 & $a$ & 0 & 0 \\
\hline$d$ & $d$ & 0 & 0 & 0 & 0 \\
\hline
\end{tabular}

\begin{tabular}{|c|c|c|c|c|c|}
\hline 5.3 & $e$ & $a$ & $b$ & $c$ & $d$ \\
\hline$e$ & $e$ & 0 & $b$ & $c$ & 0 \\
\hline$a$ & $a$ & 0 & 0 & 0 & 0 \\
\hline$b$ & 0 & 0 & 0 & $a$ & 0 \\
\hline$c$ & 0 & 0 & $-a$ & 0 & 0 \\
\hline$d$ & 0 & 0 & 0 & 0 & 0 \\
\hline
\end{tabular}

\begin{tabular}{|c|c|c|c|c|c|}
\hline $5.3^{\text {op }}$ & $e$ & $a$ & $b$ & $c$ & $d$ \\
\hline$e$ & $e$ & $a$ & 0 & 0 & 0 \\
\hline$a$ & 0 & 0 & 0 & 0 & 0 \\
\hline$b$ & $b$ & 0 & 0 & $-a$ & 0 \\
\hline$c$ & $c$ & 0 & $a$ & 0 & 0 \\
\hline$d$ & 0 & 0 & 0 & 0 & 0 \\
\hline
\end{tabular}

\begin{tabular}{|c|c|c|c|c|c|}
\hline 5.5 & $e$ & $a$ & $b$ & $c$ & $d$ \\
\hline$e$ & $e$ & 0 & $b$ & $c$ & 0 \\
\hline$a$ & $a$ & 0 & 0 & 0 & 0 \\
\hline$b$ & 0 & 0 & 0 & $a$ & 0 \\
\hline$c$ & 0 & 0 & $-a$ & 0 & 0 \\
\hline$d$ & $d$ & 0 & 0 & 0 & 0 \\
\hline
\end{tabular}

\begin{tabular}{|c|c|c|c|c|c|}
\hline $5.5^{o p}$ & $e$ & $a$ & $b$ & $c$ & $d$ \\
\hline$e$ & $e$ & $a$ & 0 & 0 & $d$ \\
\hline$a$ & 0 & 0 & 0 & 0 & 0 \\
\hline$b$ & $b$ & 0 & 0 & $-a$ & 0 \\
\hline$c$ & $c$ & 0 & $a$ & 0 & 0 \\
\hline$d$ & 0 & 0 & 0 & 0 & 0 \\
\hline
\end{tabular}

\begin{tabular}{|c|c|c|c|c|c|}
\hline 5.7 & $e$ & $a$ & $b$ & $c$ & $d$ \\
\hline$e$ & $e$ & 0 & $b$ & $c$ & $d$ \\
\hline$a$ & $a$ & 0 & 0 & 0 & 0 \\
\hline$b$ & 0 & 0 & 0 & $a$ & 0 \\
\hline$c$ & 0 & 0 & $-a$ & 0 & 0 \\
\hline$d$ & 0 & 0 & 0 & 0 & 0 \\
\hline
\end{tabular}

\begin{tabular}{|c|c|c|c|c|c|}
\hline $5.7^{o p}$ & $e$ & $a$ & $b$ & $c$ & $d$ \\
\hline$e$ & $e$ & $a$ & 0 & 0 & 0 \\
\hline$a$ & 0 & 0 & 0 & 0 & 0 \\
\hline$b$ & $b$ & 0 & 0 & $-a$ & 0 \\
\hline$c$ & $c$ & 0 & $a$ & 0 & 0 \\
\hline$d$ & $d$ & 0 & 0 & 0 & 0 \\
\hline
\end{tabular}

\begin{tabular}{|c|c|c|c|c|c|}
\hline 5.9 & $e$ & $a$ & $b$ & $c$ & $f$ \\
\hline$e$ & $e$ & 0 & $b$ & $c$ & 0 \\
\hline$a$ & $a$ & 0 & 0 & 0 & 0 \\
\hline$b$ & 0 & 0 & 0 & $a$ & 0 \\
\hline$c$ & 0 & 0 & $-a$ & 0 & 0 \\
\hline$f$ & 0 & 0 & 0 & 0 & $f$ \\
\hline
\end{tabular}

\begin{tabular}{|c|c|c|c|c|c|}
\hline $5.9^{\text {op }}$ & $e$ & $a$ & $b$ & $c$ & $f$ \\
\hline$e$ & $e$ & $a$ & 0 & 0 & 0 \\
\hline$a$ & 0 & 0 & 0 & 0 & 0 \\
\hline$b$ & $b$ & 0 & 0 & $-a$ & 0 \\
\hline$c$ & $c$ & 0 & $a$ & 0 & 0 \\
\hline$f$ & 0 & 0 & 0 & 0 & $f$ \\
\hline
\end{tabular}

\begin{tabular}{|l|l|l|l|l|l|}
\hline 5.0 & 1 & $e$ & $a$ & $b$ & $c$ \\
\hline 1 & 1 & $e$ & $a$ & $b$ & $c$ \\
\hline$e$ & $e$ & $e$ & $a$ & 0 & 0 \\
\hline$a$ & $a$ & 0 & 0 & 0 & 0 \\
\hline$b$ & $b$ & $b$ & 0 & 0 & $a$ \\
\hline$c$ & $c$ & $c$ & 0 & $-a$ & 0 \\
\hline
\end{tabular}

Demonstração. A demonstração a seguir é uma simplificação do caso mais geral que pode ser encontrada em detalhes em [6]. Seja $A$ uma álgebra alternativa mas não associativa sobre um corpo $K$ de dimensão menor que 6 e suponha que $A=S \oplus N$ é sua decomposição de Wedderburn, onde $N$ denota o radical de Jacobson, que neste caso é simplesmente o nil-radical de $A$ e $S$ é uma subálgebra semissimples. Badalov [2] demonstrou que $N$ é associativo pois é uma álgebra nilpotente de dimensão menor que 5, vamos mostrar que $\operatorname{dim}_{K} N>1$ e desse modo, $\operatorname{dim}_{K} S \leq 3$ e pelo Teorema 5.4 concluímos que $S$ é associativa. Suponha que $N=n K$, para algum $n$ e tome $a, b \in A$, 
teremos que $a n=\alpha n$ e $n b=\beta n$ para algum $\alpha, \beta \in K$ e segue daí que $(a n) b-a(n b)=0$ e então $A$ é associativa, absurdo. Isto mostra que $\operatorname{dim}_{K} S \leq 3$, em particular, pelo Teorema 5.2, $S$ é uma soma direta e finita de álgebras de matrizes sobre um anel com divisão. Como $\operatorname{dim}_{K} S=3$, nenhum $\operatorname{dos}$ fatores simples de $S$ pode ser uma álgebra de matrizes ou um anel que não é comutativo com divisão, isto é, $S$ é uma soma direta de anéis comutativos e com divisão, ou seja, corpos.

Dividiremos agora nos seguintes casos:

Caso 1: Suponha que $S$ é um corpo com idempotente $e$ e que $S \neq K e$, seja então $t \in S \backslash K e$ e denote por $K^{\prime}=K(t)$ o corpo gerado por 1 e $t$. Como $\operatorname{dim}_{K} S=\operatorname{dim}_{K^{\prime}} S \cdot \operatorname{dim}_{K} K^{\prime}$ e $\operatorname{dim}_{K} K^{\prime}>1$ temos que $S=K^{\prime}$, assim, para qualquer $a \in A$, a subálgebra gerada por $a$ e $S$ é associativa pelo Teorema de Artin.

Considere a decomposição de Peirce de $A$ em relação ao idempotente $e$ dada por $A=$ $A_{11} \oplus A_{01} \oplus A_{10} \oplus A_{00}$, temos que $S \subseteq A_{11}$ e podemos enxergar $A_{11}$ como um espaço vetorial sobre $S$, do mesmo modo $A_{10}$ e $A_{01}$ também são espaços vetoriais sobre $S$. Observe que $\operatorname{dim}_{S} A_{11}$ é um divisor próprio de $\operatorname{dim}_{K} A_{11} \leq 5$ e deste modo $\operatorname{dim}_{K^{\prime}} A_{11} \leq 2$, então $A_{11}$ é associativo. Seja $x \in A_{00}$, pelo parágrafo anterior podemos decompor $x=s+n, s \in S$ e $n \in N$. Como $S \subset A_{11}$ temos que $0=x e=s+n e \in S \oplus N$, então $s=0$ o que nos dá $x \in N$ e $A_{00} \subset N$ e $A_{00}$ também é associativo. Pelo Lema 5.2, $A_{10}$ e $A_{01}$ são distintos. Utilizando que $A_{10}, A_{11}, A_{01}$ são distintos, têm dimensão maior que ou igual a 1 sobre $S$, o que significa que têm dimensão maior que ou igual a 2 sobre $K$ e assim $\operatorname{dim}_{K}\left(A_{11}+A_{10}+A_{01}\right) \geq 2+2+2$, um absurdo, mostrando que $S=K e$.

Escrevendo $N_{i j}=A_{i j} \cap N$, observe que $A_{11}=S+N_{11}$ e que $A_{i j}=N_{i j}$ nos demais casos. Como $e$ é a identidade em $A_{11}$, um cálculo simples mostra que álgebra $A_{11}$ é associativa. Como anteriormente, temos $A_{00} \subset N$ que também é associativa. Pelo Lema 5.2, pelo menos um dos produtos $A_{10} A_{10}, A_{01} A_{01}$ não é nulo, podemos assumir, passando para a álgebra oposta se necessário, que $N_{10} N_{10} \neq 0$. Tome $b, c \in N_{10}$ com $0 \neq a=b c \in N_{01}$. Como $x_{i j}^{2}=0$ para $x_{i j} \in A_{i j}$ e $i \neq j$, segue, em particular, que os elementos $a, b$ e $c$ são linearmente independentes e como consequência $\operatorname{dim} N_{10} \geq 2$ adicionalmente. Portanto $N_{11}+N_{00}$ é nil e sua dimensão não supera 1 , assim $\left(N_{11}+N_{00}\right)^{2}=0$. Provaremos agora que

(1) $N_{11} N_{10}=N_{01} N_{11}=N_{10} N_{01}=0$

O resultado é óbvio se $N_{11}=0$, caso contrário, como $\operatorname{dim} N \leq 4$, devemos ter que $\operatorname{dim} N_{10}=2$, $\operatorname{dim} N_{01}=1$ e $N_{00}=0$, o que nos leva à decomposição $A=A_{11}+N_{10}+N_{01}$. Além disso, $N_{01}=N_{10} N_{10}$ pois $N_{01} \supseteq N_{10} N_{10}$ e ambos têm a mesma dimensão. Seja $u$ um elemento não nulo qualquer de $N_{10}$ e suponha que $u N_{10}=0$, já que $u A \neq 0$ mas $u A_{11}=0$ devemos ter que $u N_{01} \neq 0$ entretanto $u N_{01} \subseteq N_{10} N_{01}=N_{10} N_{10} N_{10} \subseteq N_{01} N_{10} \subseteq N_{00}=0$, mostrando que

(2) $u N_{10}=0$ e $u \in N_{10}$ implica $u=0$.

Em particular, se $u \in N_{11} N_{10}$, então $u N_{10} \subseteq N_{11} N_{10} N_{10} \subseteq N_{11} N_{01}=0$ concluímos então que $N_{11} N_{10}=0$ assim como a igualdade $N_{01} N_{11}=N_{10} N_{10} N_{11}=0$ e por fim que $N_{10} N_{01}=N_{10} N_{10} N_{10}=0$ o que conclui a prova de (1). De maneira análoga podemos provar ainda que $N_{10} N_{00}=N_{00} N_{01}=$ $N_{01} N_{10}=0$. 
Por fim, observe que $N_{01} N_{01}=0$, este resultado segue das propriedades dos elementos da decomposição de Peirce se $\operatorname{dim} N_{01} \leq 1$, caso contrário, $\operatorname{dim} N_{01}=2=\operatorname{dim} N_{10} \operatorname{assim} N_{10}=N_{00}=0$ e $N_{01} N_{01} N_{10} \subseteq N_{01} N_{00}=0$ o que implica em $N_{01} N_{01}=0$ por (2).

Recordando que a linearização de $x_{i j}^{2}=0$ nos fornece que $x_{i j} y_{i j}=-y_{i j} x_{i j}$ para quaisquer $y_{i j}, x_{i j} \in A_{i j} \operatorname{com} i \neq j$, e então $c b=-a$, $\operatorname{logo}$ o conjunto $\{e, a, b, c\}$ forma uma base para a subálgebra de dimensão 4 de $A$ com produtos descritos na Tabela 4.1 e com sua álgebra oposta descrita em $4.1^{o p}$.

\begin{tabular}{|c|c|c|c|c|}
\hline 4.1 & $e$ & $a$ & $b$ & $c$ \\
\hline$e$ & $e$ & 0 & $b$ & $c$ \\
\hline$a$ & $a$ & 0 & 0 & 0 \\
\hline$b$ & 0 & 0 & 0 & $a$ \\
\hline$c$ & 0 & 0 & $-a$ & 0 \\
\hline
\end{tabular}

\begin{tabular}{|c|c|c|c|c|}
\hline $4.1^{\text {op }}$ & $e$ & $a$ & $b$ & $c$ \\
\hline$e$ & $e$ & $a$ & 0 & 0 \\
\hline$a$ & 0 & 0 & 0 & 0 \\
\hline$b$ & $b$ & 0 & 0 & $-a$ \\
\hline$c$ & $c$ & 0 & $a$ & 0 \\
\hline
\end{tabular}

Se $\operatorname{dim} A=5$, então $\operatorname{dim}(S)=1 \mathrm{implica}$ em $\operatorname{dim} N=4$ e podemos tomar um quinto elemento da base que denotaremos por $d$ em algum $N_{i j}$. Se $d \in N_{11}$, temos a álgebra descrita em 5.1 e sua álgebra oposta $5.1^{o p}$ descritas abaixo.

\begin{tabular}{|c|c|c|c|c|c|}
\hline 5.1 & $e$ & $a$ & $b$ & $c$ & $d$ \\
\hline$e$ & $e$ & 0 & $b$ & $c$ & $d$ \\
\hline$a$ & $a$ & 0 & 0 & 0 & 0 \\
\hline$b$ & 0 & 0 & 0 & $a$ & 0 \\
\hline$c$ & 0 & 0 & $-a$ & 0 & 0 \\
\hline$d$ & $d$ & 0 & 0 & 0 & 0 \\
\hline
\end{tabular}

\begin{tabular}{|c|c|c|c|c|c|}
\hline $5.1^{o p}$ & $e$ & $a$ & $b$ & $c$ & $d$ \\
\hline$e$ & $e$ & $a$ & 0 & 0 & $d$ \\
\hline$a$ & 0 & 0 & 0 & 0 & 0 \\
\hline$b$ & $b$ & 0 & 0 & $-a$ & 0 \\
\hline$c$ & $c$ & 0 & $a$ & 0 & 0 \\
\hline$d$ & $d$ & 0 & 0 & 0 & 0 \\
\hline
\end{tabular}

Se $d \in N_{00}$ obtemos a álgebra 5.3 com sua respectiva álgebra oposta $5.3^{o p}$ com tabelas descritas abaixo.

\begin{tabular}{|c|c|c|c|c|c|}
\hline 5.3 & $e$ & $a$ & $b$ & $c$ & $d$ \\
\hline$e$ & $e$ & 0 & $b$ & $c$ & 0 \\
\hline$a$ & $a$ & 0 & 0 & 0 & 0 \\
\hline$b$ & 0 & 0 & 0 & $a$ & 0 \\
\hline$c$ & 0 & 0 & $-a$ & 0 & 0 \\
\hline$d$ & 0 & 0 & 0 & 0 & 0 \\
\hline
\end{tabular}

\begin{tabular}{|c|c|c|c|c|c|}
\hline $5.3^{\text {op }}$ & $e$ & $a$ & $b$ & $c$ & $d$ \\
\hline$e$ & $e$ & $a$ & 0 & 0 & 0 \\
\hline$a$ & 0 & 0 & 0 & 0 & 0 \\
\hline$b$ & $b$ & 0 & 0 & $-a$ & 0 \\
\hline$c$ & $c$ & 0 & $a$ & 0 & 0 \\
\hline$d$ & 0 & 0 & 0 & 0 & 0 \\
\hline
\end{tabular}

Se $d \in N_{01}$ obtemos a álgebra descrita em 5.5 com sua respectiva oposta $5.5^{\circ p}$. 


\begin{tabular}{|c|c|c|c|c|c|}
\hline 5.5 & $e$ & $a$ & $b$ & $c$ & $d$ \\
\hline$e$ & $e$ & 0 & $b$ & $c$ & 0 \\
\hline$a$ & $a$ & 0 & 0 & 0 & 0 \\
\hline$b$ & 0 & 0 & 0 & $a$ & 0 \\
\hline$c$ & 0 & 0 & $-a$ & 0 & 0 \\
\hline$d$ & $d$ & 0 & 0 & 0 & 0 \\
\hline
\end{tabular}

\begin{tabular}{|c|c|c|c|c|c|}
\hline $5.5^{o p}$ & $e$ & $a$ & $b$ & $c$ & $d$ \\
\hline$e$ & $e$ & $a$ & 0 & 0 & $d$ \\
\hline$a$ & 0 & 0 & 0 & 0 & 0 \\
\hline$b$ & $b$ & 0 & 0 & $-a$ & 0 \\
\hline$c$ & $c$ & 0 & $a$ & 0 & 0 \\
\hline$d$ & 0 & 0 & 0 & 0 & 0 \\
\hline
\end{tabular}

Suponha agora que $d \in N_{10}$, então $N_{10}$ tem base $\{b, c, d\}$, $\operatorname{dim} N_{01}=1$ e o produto em $N_{10}$ é da forma $u v=A(u, v) a$ onde $A(u, v)$ é uma forma alternada, assim podemos assumir que $d A(K b+$ $K c)=A(K b+K c) d=0$ e concluímos que $d N=N d=0$ e que $A$ é a álgebra descrita em 5.7 com oposta descrita em $5.7^{o p}$.

\begin{tabular}{|c|c|c|c|c|c|}
\hline 5.7 & $e$ & $a$ & $b$ & $c$ & $d$ \\
\hline$e$ & $e$ & 0 & $b$ & $c$ & $d$ \\
\hline$a$ & $a$ & 0 & 0 & 0 & 0 \\
\hline$b$ & 0 & 0 & 0 & $a$ & 0 \\
\hline$c$ & 0 & 0 & $-a$ & 0 & 0 \\
\hline$d$ & 0 & 0 & 0 & 0 & 0 \\
\hline
\end{tabular}

\begin{tabular}{|c|c|c|c|c|c|}
\hline $5.7^{\text {op }}$ & $e$ & $a$ & $b$ & $c$ & $d$ \\
\hline$e$ & $e$ & $a$ & 0 & 0 & 0 \\
\hline$a$ & 0 & 0 & 0 & 0 & 0 \\
\hline$b$ & $b$ & 0 & 0 & $-a$ & 0 \\
\hline$c$ & $c$ & 0 & $a$ & 0 & 0 \\
\hline$d$ & $d$ & 0 & 0 & 0 & 0 \\
\hline
\end{tabular}

Caso 2: Assuma agora que $S$ não é um corpo e que $A$ é uma álgebra alternativa de dimensão minimal (no máximo 5) que é deste tipo. Provaremos que a dimensão de $A$ tem que ser 5 . Sabemos que $S$ tem dois idempotentes ortogonais $e$ e $f$. Seja $A=A_{11} \oplus A_{01} \oplus A_{10} \oplus A_{00}$ a decomposição de Peirce em relação ao idempotente $e$. Como $A_{11}$ e $A_{00}$ são subálgebras próprias, se qualquer uma das duas não for associativa recai no Caso 1 por minimalidade, tem dimensão 4 e então será uma das álgebras descritas em 4.1 e $4.1^{\mathrm{op}}$. No caso em que $A_{11}$ é 4.1 obtemos a álgebra descrita em 5.9 abaixo, cuja álgebra oposta está descrita em $5.9^{\circ p}$ que também é a álgebra obtida no caso em que $A_{11}$ é como descrito em $4.1^{o p}$.

\begin{tabular}{|c|c|c|c|c|c|}
\hline 5.9 & $e$ & $a$ & $b$ & $c$ & $f$ \\
\hline$e$ & $e$ & 0 & $b$ & $c$ & 0 \\
\hline$a$ & $a$ & 0 & 0 & 0 & 0 \\
\hline$b$ & 0 & 0 & 0 & $\mathrm{a}$ & 0 \\
\hline$c$ & 0 & 0 & $-a$ & 0 & 0 \\
\hline$f$ & 0 & 0 & 0 & 0 & $f$ \\
\hline
\end{tabular}

\begin{tabular}{|c|c|c|c|c|c|}
\hline $5.9^{\text {op }}$ & $e$ & $a$ & $b$ & $c$ & $f$ \\
\hline$e$ & $e$ & $a$ & 0 & 0 & 0 \\
\hline$a$ & 0 & 0 & 0 & 0 & 0 \\
\hline$b$ & $b$ & 0 & 0 & $-a$ & 0 \\
\hline$c$ & $c$ & 0 & $a$ & 0 & 0 \\
\hline$f$ & 0 & 0 & 0 & 0 & $f$ \\
\hline
\end{tabular}

Assumindo agora que $A_{11}$ e $A_{00}$ são associativas, pelo Lema 5.2 e o fato de que $x_{i j}^{2}=0$ para $x_{i j} \in A_{i j}, i \neq j$, temos que $\operatorname{dim}\left(A_{10}+A_{01}\right) \geq 3$. Desse modo $A_{11}=K e$ e $A_{00}=K f$. Agora considere a decomposição de Peirce $A=A_{11}^{\prime} \oplus A_{01}^{\prime} \oplus A_{10}^{\prime} \oplus A_{00}^{\prime}$ em relação ao idempotente $f$, o que 
foi feito até aqui mostra que podemos assumir a igualdade $A_{11}^{\prime}=K f=A_{00}$ e $A_{00}^{\prime}=K e=A_{11}$. Dado $x_{i j} \in A_{i j}$ um cálculo simples nos fornece que $\left(e, x_{i i}, f\right)=0$ quando $i=0,1$. Além disso, $\left(e, x_{10}, f\right)=\left(e x_{10}\right) f-e\left(x_{10} f\right)=x_{10} f-x_{10} f=0$ pois $x_{10} f \in A_{10} A_{00} \subseteq A_{10}$. De forma similar $\left(e, x_{01}, f\right)=0$ e então $(e, A, f)=0$. Tome $x_{10} \in A_{10}$, então $f x_{10}=f\left(e x_{10}\right)=0$, e consequentemente $x_{10}=x_{01}^{\prime}+\alpha e \in A_{01}^{\prime}+A_{00}^{\prime}$. Note que $x_{01}^{\prime}=x_{01}^{\prime} f$, assim $0=x_{10} e=x_{01}^{\prime} e+\alpha e$. Como $x_{01}^{\prime} e \in A_{01}^{\prime} A_{00}^{\prime}=0$ tem-se $\alpha e=0$ e então $x_{10} \in A_{01}^{\prime}$ o que mostra a inclusão $A_{10} \subset A_{01}^{\prime}$. Argumentando de forma similar mostramos que $A_{10}=A_{01}^{\prime}$ e $A_{01}=A_{10}^{\prime}$. Em particular, $e+f=1$ e como isso vale para quaisquer idempotentes ortogonais, $S=K e+K f$. Observe agora que $A=K .1+P$ onde $P=K e+N$ o que mostra que $P$ é uma subálgebra própria de $A$ que não pode ser associativa, desse modo, deve ser do tipo 4.1 ou $4.1^{o p}$. Se ocorrer que esta é da forma $4.1^{o p}$ obtemos a álgebra descrita em 5.0, por outro lado, se $P$ é da forma 4.1 obtemos uma álgebra isomorfa a 5.0 e esta é isomorfa a sua oposta, concluindo a demonstração do teorema.

\begin{tabular}{|c|c|c|c|c|c|}
\hline 5.0 & 1 & $e$ & $a$ & $b$ & $c$ \\
\hline 1 & 1 & $e$ & $a$ & $b$ & $c$ \\
\hline$e$ & $e$ & $e$ & $a$ & 0 & 0 \\
\hline$a$ & $a$ & 0 & 0 & 0 & 0 \\
\hline$b$ & $b$ & $b$ & 0 & 0 & $a$ \\
\hline$c$ & $c$ & $c$ & 0 & $-a$ & 0 \\
\hline
\end{tabular}

Teorema 5.6. Uma álgebra alternativa que não é associativa e possui dimensão 5 não pode ter comprimento maior do que 3.

Demonstração. Utilizando o Teorema de Artin concluímos que se existir um conjunto gerador refinado $S$, este deve conter pelo menos três elementos que não associam pois, caso contrário, a álgebra gerada por $S$ seria associativa. Considere agora os casos:

Caso 1: Se $A$ é unitária, a cadeia deve começar por $x_{0}=1$. Pelo que foi mencionado acima temos $x_{1} \geq 4$ e então pelo Lema 2.1 devemos ter $x_{2}=5$. Isto mostra que $A$ tem comprimento limitado por 2 .

Caso 2: Vamos mostrar que se $A$ não é unitária e tem comprimento maior que 3, então deve ter comprimento 4. Seja $S$ um conjunto gerador de comprimento maior que 3 e $a_{0} \rightarrow a_{1} \rightarrow a_{2} \rightarrow$ $a_{3} \rightarrow a_{4} \rightarrow \cdots \rightarrow a_{p}$ sua cadeia. Sabemos que $a_{0}=0$ e que $a_{1} \geq 3$. Se $a_{1}=4$ então pelo Lema 2.1 devemos ter $a_{2}=5$, um absurdo. Assim, a cadeia é da forma $0 \rightarrow 3 \rightarrow a_{2} \rightarrow a_{3} \rightarrow a_{4} \rightarrow \cdots \rightarrow a_{p}$. Novamente pelo Lema 2.1 temos que $a_{2} \geq 4$. Se $a_{2}=5$ teríamos que $l(S)=2$, portanto $a_{2}=4$. Se $a_{4}=a_{2}$ então devemos ter $a_{i}=2$ para todo $i>2$, contrariando a hipótese de $S$ ser gerador. Desse modo, a única cadeia possível é dada por $0 \rightarrow 3 \rightarrow 4 \rightarrow 4 \rightarrow 5$, que tem comprimento 4 .

Suponha que $A$ não é unitária e que $l(A)>3$. Fixe um conjunto gerador $S$ de comprimento 4 , cuja cadeia é $0 \rightarrow 3 \rightarrow 4 \rightarrow 4 \rightarrow 5$. Tomamos então $S=\{x, y, z\}$. Deve haver uma palavra irredutível 
$n$ de comprimento 4, como não existem palavras irredutíveis de comprimento 3 e apenas uma palavra $w$ de comprimento 2, esta deve ser da forma $n=w^{2}$. Se $w=x^{2}$ (os casos em que $w=y^{2}$ e $w=z^{2}$ são análogos) devemos ter que $n=w w=(x x)(x x)=x(x(x x))$ pois $A$ é alternativa. Por outro lado, não existem palavras irredutíveis de comprimento 3 o que implica em $x(x x) \in\left\langle x, y, z, x^{2}\right\rangle$. Agora, multiplicando à esquerda por $x$ temos que $n=x(x(x x)) \in\left\langle x^{2}, x y, x z, x x^{2}\right\rangle \subset\left\langle x, y, z, x^{2}\right\rangle \subset \mathcal{L}_{2}(S)$ onde a inclusão segue do fato de $x y, x z, x(x x)$ não serem palavras irredutíveis, uma contradição.

Se $w=x y$ (os demais casos são análogos), então $n=(x y)(x y)=x(y(x y))$ prosseguimos de modo semelhante ao caso $w=x^{2}$ e obtemos $y(x y) \in\langle x, y, z, x y\rangle$ e assim $x(y(x y)) \in\left\langle x^{2}, x y, x z, x(x y)\right\rangle \subset$ $\langle x, y, z, x y\rangle \subset \mathcal{L}_{2}(S)$, um absurdo.

Com isso mostramos que para qualquer álgebra alternativa $A$, a relação $l(A) \leq 3$ é verdadeira.

Conhecer os valores dos comprimentos que podem ser realizados por uma álgebra é tão importante quanto conhecer os conjuntos geradores. No Capítulo 3 caracterizamos as bases derivadas a partir de um conjunto gerador, o teorema a seguir exibe bases para uma álgebra alternativa que não é unitária e realiza o comprimento 3.

Teorema 5.7. Seja A uma álgebra alternativa de dimensão 5, que não é unitária e que tem comprimento 3. Então um conjunto gerador é da forma $S=\{x, y, z\}$ e uma base é da forma $\left\{x, y, z, x^{2}, x^{3}\right\},\{x, y, z, x y,(x y) z\}$, $\{x, y, z, x y, z(x y)\}$.

Demonstração. Fixe um conjunto gerador $S$ de comprimento 3. Começamos observando que a única cadeia possível é dada por $0 \rightarrow 3 \rightarrow 4 \rightarrow 5$, desse modo, existem $x, y, z \in A$ tais que $\{x, y, z, w\}$ é base de $\mathcal{L}_{2}(S)$ onde $l(x)=l(y)=l(z)=1$ e $l(w)=2$. Isto significa que $S=\{x, y, z\}$ e $w$ pertence ao conjunto $\left\{x^{2}, y^{2}, z^{2}, z x, x z, y x, x y, y z, z y\right\}$. Iremos considerar os casos $w=x^{2}$ e $w=x y$ e os demais podem ser tratados por um raciocínio semelhante.

Caso 1: Vale a igualdade $w=x^{2}$. A palavra irredutível $w^{\prime}$ de comprimento 3 que completa a base satisfaz a relação $w^{\prime} \in\left\{x^{2} y, y x^{2}, x^{3}, z x^{2}, x^{2} z\right\}$. Vamos mostrar que qualquer que seja $w^{\prime}$, devemos ter que $x^{3}$ também é uma palavra irredutível.

Caso 1.1: Quando $w^{\prime} \in\left\{x^{2} y, x^{2} z\right\}$, a igualdade $(x x) y=x(x y)$ e o fato de que $x z, x y \in$ $\left\langle x, y, z, x^{2}\right\rangle$ implicam em $(x x) y=x(x y)=x\left(k_{1} x+k_{2} y+k_{3} z+k_{4} x^{2}\right)$ com $k_{i} \in K$. Assim, se $x^{3}$ não fosse irredutível, então $x^{2} y \in \mathcal{L}_{2}(S)$, o que seria uma contradição. O caso em que $w=x^{2} z$ é análogo.

Caso 1.2: Se $w^{\prime} \in\left\{y x^{2}, z x^{2}\right\}$, as relações $z(x x)=(z x) x$ e $z x \in\left\langle x, y, z, x^{2}\right\rangle$ implicam em $z x^{2}=(z x) x=\left(k_{1} x+k_{2} y+k_{3} z+k_{4} x^{2}\right) x$ com $k_{i} \in K$. Assim, se $x^{3}$ não fosse irredutível, então $z x^{2} \in \mathcal{L}_{2}(S)$, o que seria uma contradição. O caso $w^{\prime}=y x^{2}$ é análogo.

Isto mostra que se $w=x^{2}$ é irredutível, então é suficiente procurar por uma palavra irredutível na forma $x^{3}$. Além disso, quando $x^{3}$ não é uma palavra irredutível, então não há uma palavra 
irredutível de comprimento 3.

Caso 2: Vale a relação $w=x y$. Uma palavra irredutível $w^{\prime}$ de comprimento 3 deve ser da forma $w^{\prime} \in\{(x y) x, x(x y), y(x y),(x y) y, z(x y),(x y) z\}$. Vamos mostrar que as quatro primeiras possibilidades não podem ser palavras irredutíveis. Utilizamos o mesmo raciocínio dos Casos 1.1 e 1.2 e obtemos as seguintes proposições:

I. $x^{2} y$ é irredutível se, e somente se, $x y^{2}$ é irredutível;

II. Se $x y x$ é irredutível, então $x^{2} y$ é irredutível;

III. Se $y x y$ é irredutível, então $x y^{2}$ é irredutível.

Vamos mostrar que podemos assumir que $y^{3}$ não é irredutível. Se $y^{3}$ for irredutível, então o conjunto $B=\left\{x, y, z, x y, y^{3}\right\}$ é linearmente independente. Por um lado, se $y^{2}=a x+b y+c z$ para alguns $a, b, c \in K$, então $y^{3}=a(x y)+b y^{2}+c(z y) \in\langle x, y, z, x y\rangle$ o que contraria o fato de $B$ ser um conjunto linearmente independente. Por outro lado, se $y^{2} \notin\langle x, y, z\rangle$, então o conjunto $\left\{x, y, z, y^{2}\right\}$ é linearmente independente e recaímos no Caso 1.

Assumindo então que $y^{2} y$ não é uma palavra irredutível, devemos ter que $x y^{2}$ também não pode ser, com efeito, escrever $y^{2}$ como combinação linear de $x, y, z, x y$ e considerar os elementos $L_{x}\left(y^{2}\right)$ e $R_{y}\left(y^{2}\right)$. Como consequência do item I, $x^{2} y, x y^{2}$ não são irredutíveis e a contrapositiva dos itens II e III fornecem que as demais também não. Assim, podemos concluir que pelo menos um dos elementos $z(x y),(x y) z$ deve completar a base.

Com base no Teorema 5.7 podemos verificar quais álgebras do Teorema 5.5 satisfazem as condições prescritas. Podemos reduzir o número de casos a serem tratados através da proposição a seguir.

Proposição 5.1. Seja $A$ uma $K$-álgebra de comprimento $m$, então $l\left(A^{o p}\right)=m$.

Demonstração. Basta observar que se $S$ é um conjunto gerador de $A$, então $S_{n} \cap A^{o p}=S_{n} \cap A$ e então $S$ é gerador em ambas as álgebras e possui mesmo comprimento.

Vamos analisar as álgebras apresentadas nas tabelas do Teorema 5.5 e determinar seus comprimentos, em virtude da Proposição 5.1, é suficiente tratar apenas uma das álgebras dentre $A$ e $A^{\text {op }}$.

Álgebra 4.1: Considerando a adjunção de elemento identidade a esta álgebra, obtemos que $l(A) \leq 3$, pelo Teorema 5.6. Seja $S$ um conjunto gerador refinado, o Teorema de Artin garante que $|S| \geq 3$. Desse modo, as únicas cadeias possíveis para $S$ são $1 \rightarrow 4 \rightarrow 5$ ou $1 \rightarrow 5$. Para concluir que $l(A)=2$ tome o conjunto $S=\{e, b, c\}$.

Para analisarmos o caso das álgebras de dimensão 5 , utilizaremos a notação de coordenadas em relação à base $\{e, a, b, c, d\}$ para elementos arbitrários $x=\left(a_{1}, a_{2}, a_{3}, a_{4}, a_{5}\right), y=\left(b_{1}, b_{2}, b_{3}, b_{4}, b_{5}\right)$ e $z=\left(z_{1}, z_{2}, z_{3}, z_{4}, z_{5}\right)$ de $A$. Note que em todos os casos temos que $l(A)>1$. Com efeito, para as álgebras 5.1,5.3, 5.5,5.7 e 5.9 que não são unitárias temos que $a=b c \notin\langle b, c\rangle$ enquanto para a álgebra 
5.0 vale que $a=b c \notin\langle 1, b, c\rangle$ e pela Proposição 3.1 segue que $l(A)>1$.

Vamos determinar o comprimento das álgebras do Teorema 5.5.

Álgebra 5.1: As seguintes relações são verdadeiras para quaisquer $x, y \in A$

(1) $x y=a_{1} y+\left(a_{2} b_{1}-a_{1} b_{2}+a_{3} b_{4}-a_{4} b_{3}\right) a+\left(a_{5} b_{1}\right) d \in\langle y, a, d\rangle$;

(2) $x a=0, a x=a_{1} a$ e então $a x, x a \in\langle a\rangle$;

(3) $d x=a_{1} d, x d=a_{1} d$ e então $d x, x d \in\langle d\rangle$.

Vamos mostrar que esta álgebra não possui nenhuma das bases explicitadas pelo Teorema 5.7. Seja $S=\{x, y, z\}$ um conjunto gerador de comprimento 3 .

Note que $x^{2}=a_{1} x+\left(a_{5} a_{1}\right) d$ e por (3) temos $x d, d x \in\langle d\rangle$. Isto mostra que $x, x^{2}, x^{3}$ pertencem ao espaço gerado por $x$ e $d$, assim o conjunto $\left\{x, y, z, x^{2}, x^{3}\right\}$ é linearmente dependente. Escrevemos $x y=a_{1} y+\left(a_{2} b_{1}-a_{1} b_{2}+a_{3} b_{4}-a_{4} b_{3}\right) a+\left(a_{5} b_{1}\right) d=a_{1} y+m a+n d$ e supomos que o conjunto $\{x, y, z, x y\}$ é linearmente independente.

Para o que falta, precisaremos do seguinte resultado.

Lema 5.5. Se $a \in\langle x, y, z, x y\rangle$ ou $d \in\langle x, y, z, x y\rangle$, então os conjuntos $\{x, y, z, x y, z(x y)\},\{x, y, z, x y,(x y) z\}$ não são uma base de $A$. Em particular, $a$ e $d$ não podem pertencer simultaneamente a $\langle x, y, z, x y\rangle$.

Demonstração. Provaremos o caso em que $a \in\langle x, y, z, x y\rangle$, o segundo é análogo. Suponha que $a \in\langle x, y, z, x y\rangle$. Por (1) podemos escrever $x y=k_{1} y+k_{2} a+k_{3} d$ com $k_{i} \in K$ e concluir que $k_{3}=0$ ou $d \in\langle x, y, z, x y\rangle$. Se $k_{3}=0$ temos $x y=k_{1} y+k_{2} a$ de onde seguem as igualdades $z(x y)=k_{1}(z y)$ e $(x y) z=k_{1}(y z)+k_{2}(a z)$. Como $a, z y, y z \in\langle x, y, z, x y\rangle$, temos que $z(x y),(x y) z$ não são palavras irredutíveis. Se $k_{3} \neq 0$, escrevemos $\frac{1}{k_{3}}\left(x y-k_{1} y-k_{2} a\right)=d$ e então $d \in\langle x, y, z, x y\rangle$. Como as palavas $z(x y)$ e $(x y) z$ dependem apenas de $z, x y, a, d$ que são elementos de $\langle x, y, z, x y\rangle$ concluímos que não completam uma base.

Isto mostra que $m$ e $n$ não podem ser ambos nulos. Com efeito, se $m=0$ ou $n=0$ teríamos que $d \in\langle x, y, z, x y\rangle$ ou $a \in\langle x, y, z, x y\rangle$ e então $S$ não teria comprimento 3. Assim, podemos supor que $m$ e $n$ são ambos não nulos. Além disso, $a_{5} a_{1}=0$, pois como $x^{2}=a_{1} x+\left(a_{5} a_{1}\right) d$, se $a_{5} a_{1} \neq 0$, então $d \in\left\langle x^{2}, x\right\rangle \subset\langle x, y, z, x y\rangle$, o que seria uma contradição. De modo análogo, deduzimos que $b_{5} b_{1}=0$. Além disso, temos que $x y+y x=a_{1} y+b_{1} x+\left(a_{5} b_{1}+b_{5} a_{1}\right) d$ o que implica em $\left(a_{5} b_{1}+b_{5} a_{1}\right)=0$.

Se $a_{5}=0$, então $n=0$, e decorre de (1) que $a \in\langle x, y, z, x y\rangle$, o que novamente é uma contradição. Assim, como $a_{5} a_{1}=0$, devemos ter $a_{1}=0$, pois $a_{5} \neq 0$, consequentemente $\left(a_{5} b_{1}+b_{5} a_{1}\right)=a_{5} b_{1}=$ 0 e assim $b_{1}=0$. Como $b_{1}=0$ implica em $n=0$ concluímos que em quaisquer casos, $S$ satisfaz as hipóteses do Lema 5.5 e então $l(S)=2$ pois já sabíamos que $l(A)>1$. Um conjunto gerador de comprimento dois é $S=\{e, b, c, d\}$.

Álgebra 5.3: As seguintes relações são verdadeiras para quaisquer $x, y \in A$

(1) $x y=a_{1} y+\left(a_{2} b_{1}-a_{1} b_{2}+a_{3} b_{4}-a_{4} b_{3}\right) a-\left(a_{1} b_{5}\right) d \in\langle y, a, d\rangle$; 
(2) $x a=0, a x=a_{1} a$ e então $a x, x a \in\langle a\rangle$;

(3) $d x=0=x d$ e então $d x, x d \in\langle d\rangle$.

Novamente, supomos que existe um conjunto gerador $S=\{x, y, z\}$ que possui comprimento 3 . Observe que $x^{2}=a_{1} x-\left(a_{1} a_{5}\right) d$ e então $x, x^{2}, x^{3} \in\langle x, d\rangle$, em particular, $\left\{x, y, z, x^{2}, x^{3}\right\}$ não é base de $A$. Escrevemos $x y=a_{1} y+\left(a_{2} b_{1}-a_{1} b_{2}+a_{3} b_{4}-a_{4} b_{3}\right) a-\left(a_{1} b_{5}\right) d=a_{1} y+m a+n d$ e supomos que o conjunto $\{x, y, z, x y\}$ é linearmente independente.

Destacamos que o Lema 5.5 também é verdadeiro para esta álgebra e como consequência temos que $x y=a_{1} y+n a+m d$ com $n, m \neq 0$. Pelo mesmo raciocínio utilizadas na álgebra 5.1 concluímos que, $a_{5} a_{1}=0, b_{1} b_{5}=0$ e que $x y+y x=a_{1} y+b_{1} x-\left(a_{1} b_{5}+b_{1} a_{5}\right) d$ portanto $\left(a_{1} b_{5}+b_{1} a_{5}\right)=0$.

Se $a_{1}=0$, então $n=0$, e decorre de (1) que $a \in\langle x, y, z, x y\rangle$, o que novamente é uma contradição. Assim, como $a_{1} a_{5}=0$, devemos ter $a_{5}=0$, pois $a_{1} \neq 0$, consequentemente $\left(a_{5} b_{1}+b_{5} a_{1}\right)=b_{5} a_{1}=$ 0 e assim $b_{5}=0$. Como $b_{5}=0$ implica em $n=0$ concluímos que em quaisquer casos, $S$ satisfaz as hipóteses do Lema 5.5 e então $l(S)=2$ pois já sabíamos que $l(A)>1$. Um conjunto gerador de comprimento dois é $S=\{e, b, c, d\}$.

Álgebra 5.5: As seguintes relações são verdadeiras para quaisquer $x, y \in A$;

(1) $x y=a_{1} y+\left(a_{2} b_{1}+a_{3} b_{4}-a_{4} b_{3}-a_{1} b_{2}\right) a+\left(a_{5} b_{1}-a_{1} b_{5}\right) d \in\langle y, a, d\rangle$;

(2) $a x=a_{1} a, x a=0$ e então $a x, x a \in\langle a\rangle$;

(3) $d x=a_{1} d, x d=0$ e então $d x, x d \in\langle d\rangle$.

Suponha que existe um conjunto gerador $S=\{x, y, z\}$ de comprimento 3. Observe que $x^{2}=a_{1} x$ então uma base não é da forma $\left\{x, y, z, x^{2}, x^{3}\right\}$. Escrevemos $x y=a_{1} y+\left(a_{2} b_{1}-a_{1} b_{2}+a_{3} b_{4}-\right.$ $\left.a_{4} b_{3}\right) a-\left(a_{1} b_{5}\right) d=a_{1} y+m a+n d$ e supomos que o conjunto $\{x, y, z, x y\}$ é linearmente independente.

Novamente, pelo Lema 5.5 podemos supor que $x y=a_{1} y+m a+n d$ com $m, n \neq 0$, em particular, $m a+n d \in\langle x, y, z, x y\rangle$. Por um lado, $z(x y)=z\left(a_{1} y+m a+n d\right)=a_{1}(z y) \in\langle x, y, z, x y\rangle$, onde na última igualdade utilizamos (2) e (3). Por outro, novamente por (2) e (3), temos $(x y) z=$ $\left(a_{1} y+m a+n d\right) z=a_{1}(y z)+z_{1}(n a+p d)$. Como $y z, m a+n d \in\langle x, y, z, x y\rangle$, concluímos que as palavras $z(x y),(x y) z$ não são irredutíveis e assim $l(A)=2$ pois já sabíamos que $l(A)>1$.

Álgebra 5.7: As seguintes relações são verdadeiras para quaisquer $x, y \in A$;

(1) $x y=a_{1} y+\left(a_{2} b_{1}-a_{1} b_{2}+a_{3} b_{4}-a_{4} b_{3}\right) a \in\langle y, a\rangle$;

(2) $x a=0, a x=a_{1} a$ e então $a x, x a \in\langle a\rangle$;

(3) $d x=0, x d=a_{1} d$ e então $d x, x d \in\langle d\rangle$.

Suponha que existe um conjunto gerador $S=\{x, y, z\}$ de comprimento 3. Observe que $x^{2}=k_{1} x$ e então uma base não é da forma $\left\{x, y, z, x^{2}, x^{3}\right\}$. Escrevemos $x y=a_{1} y+m a$ e supomos que o conjunto $\{x, y, z, x y\}$ é linearmente independente. Se $m=0$ então $x y=a_{1} y$ e o conjunto $\{x, y, z, x y\}$ é 
linearmente dependente. Podemos então supor que $m \neq 0$ e, consequentemente $\frac{1}{m}\left(x y-a_{1} y\right)=a$ e concluímos que $a \in\langle x, y, z, x y\rangle$. Segue de (1) que $z(x y)=a_{1}(z y)$ e $(x y) z=a_{1}(y z)+\left(m z_{1}\right) a$. Como $a, z y, y z \in\langle x, y, z, x y\rangle$ concluímos que $z(x y)$ e $(x y) z$ não são palavras irredutíveis. Consequentemente, esta álgebra possui comprimento 2 pois já sabíamos que $l(A)>1$. Um conjunto gerador de comprimento dois é $S=\{e, b, c, d\}$.

Álgebra 5.9: As seguintes relações são verdadeiras para quaisquer $x, y \in A$;

(1) $x y=a_{1} y+\left(a_{2} b_{1}-a_{1} b_{2}+a_{3} b_{4}-a_{4} b_{3}\right) a+\left(a_{5} b_{5}-a_{1} b_{5}\right) f \in\langle y, a, f\rangle$;

(2) $a x=a_{1} a, x a=0$ e então $a x, x a \in\langle a\rangle$;

(3) $f x=x f=a_{5} f$ e então $f x, x f \in\langle f\rangle$.

Suponha que existe um conjunto gerador $S=\{x, y, z\}$ de comprimento 3. Observe que por (1) temos que $x^{2}=a_{1} x+\left(a_{5}^{2}-a_{1} a_{5}\right) f$ o que implica em $x, x^{2}, x^{3} \in\langle x, f\rangle$ e então uma base não pode ser da forma $\left\{x, y, z, x^{2}, x^{3}\right\}$. Escrevemos $x y=a_{1} y+\left(a_{2} b_{1}-a_{1} b_{2}+a_{3} b_{4}-a_{4} b_{3}\right) a+\left(a_{5} b_{5}-a_{1} b_{5}\right) f=$ $a_{1} y+m a+n f$ e supomos que o conjunto $\{x, y, z, x y\}$ é linearmente independente.

O seguinte lema é o caso análogo ao Lema 5.5 e será útil.

Lema 5.6. Se $a \in\langle x, y, z, x y\rangle$ ou $f \in\langle x, y, z, x y\rangle$, então os conjuntos $\{x, y, z, x y, z(x y)\}$ e $\{x, y, z, x y,(x y) z\}$ não são uma base de $A$. Em particular, $a$ e $f$ não podem pertencer simultaneamente a $\langle x, y, z, x y\rangle$.

Demonstração. Provaremos o caso em que $f \in\langle x, y, z, x y\rangle$, o segundo é análogo. Suponha que $f \in\langle x, y, z, x y\rangle$. Como $x y=a_{1} y+m a+n f$, podemos concluir que $m=0$ ou $a \in\langle x, y, z, x y\rangle$. Se $m=0$ temos que $x y=a_{1} y+n f$ e por (3) seguem as igualdades $z(x y)=a_{1}(z y)+\left(n z_{5}\right) f$ e $(x y) z=$ $a_{1}(y z)+\left(n z_{5}\right) f$. Como $z y, y z, f \in\langle x, y, z, x y\rangle$, temos que $z(x y),(x y) z$ não são palavras irredutíveis. Se $m \neq 0$, escrevemos $\frac{1}{m}\left(x y-a_{1} y-n f\right)=a$ e então $a \in\langle x, y, z, x y\rangle$. Como as palavas $z(x y)$ e $(x y) z$ dependem apenas de $z y, y z, a, f$ por (2) e (3), que são elementos de $\langle x, y, z, x y\rangle$ concluímos que $z(x y),(x y) z$ não são palavras irredutíveis.

Pelo Lema 5.6, podemos escrever $x y=a_{1} y+m a+n f$ com $m, n \neq 0$. Além disso, os coeficientes do elemento $f$ nos produtos $x^{2}=a_{1} x+\left(a_{5}^{2}-a_{1} a_{5}\right) f, y^{2}=b_{1} y+\left(b_{5}^{2}-b_{1} b_{5}\right) f$ e $x y+y x=a_{1} y+b_{1} x+$ $\left(2 a_{5} b_{5}+-b_{1} a_{5}-a_{1} b_{5}\right) f$ devem se anular, do contrário, teremos que $f \in\langle x, y, z, x y\rangle$ e a conclusão segue do Lema 5.6. Isto mostra que devemos ter $-b_{1} a_{5}-a_{1} b_{5}+2 a_{5} b_{5}=0,-b_{1} b_{5}+b_{5}^{2}=0,-a_{1} a_{5}+a_{5}^{2}=0$.

Se $b_{5}=0$, então $n=0$ o que implica em $a \in\langle x, y, z, x y\rangle$ e a conclusão segue pelo Lema 5.6. Assim, $b_{5} \neq 0$ e por $-b_{1} b_{5}+b_{5}^{2}=0$ temos que $b_{1}=b_{5}$. Substituindo $b_{1}=b_{5}$ em $2 a_{5} b_{5}-b_{1} a_{5}-a_{1} b_{5}=0$ obtemos $a_{5} b_{5}-a_{1} b_{5}=0$ e como $b_{5} \neq 0$ temos que $a_{5}=a_{1}$. Concluímos então que $b_{1}=b_{5}$ o que implica em $a_{5}=a_{1}$. Substituindo $a_{5}=a_{1}$ e $b_{5}=b_{1} \mathrm{em}-a_{1} b_{5}+a_{5} b_{5}=a_{1} b_{1}+a_{1} b_{5}=0$ temos que $n=0$, e o resultado segue pelo Lema 5.6. Isto mostra que $l(A)=2$ pois já sabíamos que $l(A)>1$. Um conjunto gerador de comprimento dois é dado por $S=\{e, b, c, f\}$.

Álgebra 5.0: Pelo Teorema 5.6 concluímos que $l(A) \leq 3$. Como a álgebra 5.0 é alternativa mas não é associativa, um conjunto gerador refinado deve ter pelo menos três elementos que não associam. Assim, considerando uma cadeia de $S$, devemos ter $x_{1}=4$ e pelo Lema 2.1 segue que 
$x_{2}=5$, portanto $l(A) \leq 2$. Para mostrar a igualdade, considere o conjunto $S=\{e, b, c\}$.

Por fim, condensamos os resultados obtidos na seguinte proposição.

Proposição 5.2. Se $A$ é uma $K$-álgebra alternativa e que tem dimensão menor que 6 sobre um corpo perfeito $K$, então são verdadeiras as seguintes afirmações:

1. Se $A$ tem dimensão 5 e não é associativa:

- A tem comprimento 2 se, e somente se, é isomorfa a uma das álgebras listadas no Teorema 5.5.

2. Se $A$ não é associativa e tem dimensão 4 :

- O comprimento de $A$ não excede 2;

- A tem comprimento 2 se, e somente se, é isomorfa a 4.1 ou $4.1^{\text {op }}$;

- A é unitária e tem comprimento 1 se, e somente se, para quaisquer $x, y \in A$, vale a relação $x y \in\langle 1, x, y\rangle$;

- A não é unitária e tem comprimento 1 se, e somente se, para quaisquer $x, y \in A$ vale a relação $x y \in\langle x, y\rangle$.

3. Em dimensão 3 e 2:

- Se $A$ não é associativa, então $l(A)=1$;

- $A$ é unitária e tem comprimento 1 se, e somente se, para quaisquer $x, y \in A$, vale a relação $x y \in\langle 1, x, y\rangle$;

- $A$ não é unitária e tem comprimento 1 se, e somente se, para quaisquer $x, y \in A$ vale a relação $x y \in\langle x, y\rangle$.

\subsection{O comprimento dos Octônios}

Vamos considerar o seguinte exercício. Seja $A$ uma álgebra unitária de dimensão 8 que é alternativa mas não é associativa. Quantos elementos pode ter um conjunto gerador refinado $S$ para que se possa ter $l(A)>3$ ? Em uma primeira análise, sabemos que devemos ter $8 \geq a_{4}=$ $a_{1}+\left(a_{2}-a_{1}\right)+\left(a_{4}-a_{2}\right) \geq(|S|+1)+1+1=|S|+3$ e assim $5 \geq|S| \geq 3$.

Os Octônios, que serão denotados por $\mathrm{O}$, formam uma álgebra unitária que não é associativa e que possui dimensão 8 sobre $\mathbb{R}$ cuja tabela de multiplicação é dada a seguir. 


\begin{tabular}{|c|c|c|c|c|c|c|c|c|}
\hline$*$ & 1 & $i$ & $j$ & $k$ & $l$ & $i l$ & $j l$ & $k l$ \\
\hline 1 & 1 & $i$ & $j$ & $k$ & $l$ & $i l$ & $j l$ & $k l$ \\
\hline$i$ & $i$ & -1 & $k$ & $-j$ & $i l$ & $-l$ & $-k l$ & $j l$ \\
\hline$j$ & $j$ & $-k$ & -1 & $i$ & $j l$ & $k l$ & $-l$ & $-i l$ \\
\hline$k$ & $k$ & $j$ & $-i$ & -1 & $k l$ & $-j l$ & $i l$ & $-l$ \\
\hline$l$ & $l$ & $-i l$ & $-j l$ & $-k l$ & -1 & $i$ & $j$ & $k$ \\
\hline$i l$ & $i l$ & $l$ & $-k l$ & $j l$ & $-i$ & -1 & $-k$ & $j$ \\
\hline$j l$ & $j l$ & $k l$ & $l$ & $-i l$ & $-j$ & $k$ & -1 & $-i$ \\
\hline$k l$ & $k l$ & $-j l$ & $i l$ & $l$ & $-k$ & $-j$ & $i$ & -1 \\
\hline
\end{tabular}

Os Octônios foram descobertos por John Graves [11], em 1843, e dois anos depois foram redescobertos por Arthur Cayley [3].

Podemos mostrar diretamente que os Octônios não possuem divisores de zero e que todo elemento que não é nulo possui um inverso. Fixado $x=a_{0}+a_{1} i+a_{2} j+a_{3} k+\ldots+a_{7} k l \operatorname{com} a_{i} \in \mathbb{R}$ e nem todos $a_{i}$ nulos. Denotamos por $\operatorname{Re}(x)$ o coeficiente $a_{0}$ e definimos $\operatorname{Im}(x)$ por $\operatorname{Im}(x)=$ $\operatorname{Re}(x)-x$. Desse modo, o inverso do elemento $x$, denotado por $x^{-1}$ é dado por

$$
x^{-1}=(\operatorname{Re}(x)-\operatorname{Im}(x)) /\left(a_{0}^{2}+\ldots+a_{k}^{2}\right)
$$

Considerando os Octônios como álgebra unitária sobre os reais e $S$ um conjunto gerador com três elementos, os demais casos podem ser tratados de forma análoga. É possível que se tenha $a_{2}-a_{1}=1$ ? Para responder esta questão enunciaremos um teorema e listaremos algumas de suas consequências.

Teorema 5.8 (Frobenius). [18] Seja A uma $\mathbb{R}$-álgebra de dimensão finita, associativa e com divisão. Então A é isomorfa a álgebra dos números Reais, Complexos ou Quatérnios.

Das álgebras mencionadas no Teorema de Frobenius, as álgebras dos números reais e complexos são comutativas e possuem dimensão 1 e 2, respectivamente, enquanto os Quatérnios não são comutativos e possuem dimensão 4 .

Proposição 5.3. Se $x \in \mathbb{O}$ então $x^{2} \in\langle 1, x\rangle$.

Demonstração. Se o conjunto $\left\{1, x, x^{2}\right\}$ fosse linearmente independente, a álgebra gerada por $x$ seria uma álgebra com divisão, comutativa e de dimensão superior a 2, o que contradiz o Teorema de Frobenius.

A Proposição 5.3 nos diz que uma palavra irredutível nunca é potência de uma outra, fato que será usado sem menção explícita nos procedimentos abaixo.

Proposição 5.4. Se $x \in \mathbb{O}$ então existem $k_{1}, k_{2} \in \mathbb{R}$ tais que $x^{-1}=k_{1}+k_{2} x$.

Demonstração. Pela proposição anterior temos que $x^{2}=t_{1}+t_{2} x$ com $t_{1}, t_{2} \in \mathbb{R}$. Multiplicando ambos os lados por $x^{-1}$ temos que $x=t_{1} x^{-1}+t_{2}$ assim, se $t_{1}=0$ temos que $x$ é unidade; caso contrário, $t_{1} \neq 0$ e podemos escrever $t_{1}^{-1} x-t_{2} t_{1}^{-1}=x^{-1}$. 
Se $x^{2}=t x$, então $x=\left(x^{2}\right) x^{-1}=(t x) x^{-1}$ e então $x \in \mathbb{R}$. Vamos destacar duas consequências úteis da Proposição 5.4:

- Sejam $S$ um conjunto gerador refinado e $x, y \in S$. Se o produto $x y$ pertence ao espaço gerado por $\{1, x, y\}$ então vale que $x y=a_{1}+a_{2} x+a_{3} y$. Com efeito, se $x y=k_{1}+k_{2} x+k_{3} y$ então $\left(x-k_{3}\right) y=k_{1}+k_{2} x$, escrevemos $\left(x-k_{3}\right)^{-1}=a+b x$ e obtemos $y=(a+b x)\left(k_{1}+k_{2} x\right)=$ $\left(a k_{1}\right)+\left(a k_{2}+b k_{1}\right) x+\left(b k_{2}\right) x^{2} \in\langle 1, x\rangle$. Consequentemente existem escalares $c_{1}, c_{2}$ tais que $y=c_{1}+c_{2} x$. Em particular, o conjunto $S \backslash\{y\}=R \subset S$ ainda é gerador, além disso, pelo Corolário 2.2 vale que $l(R) \geq l(S)$. Com efeito, escrevemos $S=\left\{x, y, x_{1}, \ldots, x_{s}\right\}$ e provamos por indução que $\left\langle S_{n}\right\rangle$ está contido em $\left\langle R_{m}\right\rangle$ para algum $m \geq n$. Observamos que é suficiente mostrar que as palavras irredutíveis em $S_{n}$ pertencem ao espaço gerado por $R_{m}$ pois assim este deverá conter uma base de $\left\langle S_{n}\right\rangle$. Prosseguimos por indução no comprimento das palavras irredutíveis.

Caso base: Se $w$ é uma palavra irredutível em $S_{2}$ cujos fatores não envolvem o elemento $y$, então $w \in R_{2} \subset\left\langle R_{2}\right\rangle$. Por outro lado, se $w=$ ay para algum $a \in S$ temos que $w=a y=$ $a\left(c_{1}+c_{2} x\right)=c_{1} a+c_{2}(a x)$ que pertence a $\left\langle R_{2}\right\rangle$ pois $a$ e $x$ pertencem a $R$.

Hipótese de indução: Suponha que toda palavra irredutível de comprimento inferior a $n$ pertença a $\left\langle R_{s}\right\rangle$ para algum $s$.

Passo indutivo: Seja $w$ uma palavra irredutível de $S$ e de comprimento $n$, podemos escrever $w=w_{1} w_{2} \operatorname{com} w_{1}$ e $w_{2}$ irredutíveis. Pela hipótese de indução $w_{1}, w_{2} \in\left\langle R_{s}\right\rangle$ e então $w_{1} w_{2}$ é combinação linear de palavras cujos comprimentos não excedem o valor $2 s$, isto é, $w \in\left\langle R_{2 s}\right\rangle$.

- Fixando $S=\left\{x_{1}, \ldots, x_{m}\right\}$ e $R=S \backslash\left\{x_{1}\right\}$. Podemos argumentar de modo similar ao caso anterior para concluir que se $x_{1} \in\left\langle R_{i}\right\rangle$ para algum $i \geq 2$, então $R$ também é um conjunto gerador e satisfaz $l(R) \geq l(S)$.

Podemos generalizar a última observação através do lema a seguir.

Lema 5.7. Seja $\left\{x_{1}, \ldots, x_{n}\right\}=S \subset \mathrm{O}$ um conjunto gerador refinado. Se existe um inteiro positivo $k$ tal que $x_{1} \in \mathcal{L}_{k}(R)$, onde $R=S \backslash\left\{x_{1}\right\}$, então $R$ é um conjunto gerador e $l(R) \geq l(S)$.

Demonstração. Uma vez provado que $R$ também é um conjunto gerador, podemos utilizar o Corolário 2.2 para concluir que $l(R) \geq l(S)$. Vamos demonstrar que para cada $n>0$ existe um inteiro positivo $m$ tal que as palavras irredutíveis em $S_{n}$ pertencem a $\left\langle R_{m}\right\rangle$. Tomando $n=l(S)$ deve existir um índice $m^{\prime}$ tal que $\left\langle R_{m^{\prime}}\right\rangle$ contém todas as palavras irredutíveis de comprimento $l(S)$. Observamos que $\left\langle R_{m}\right\rangle \subset\left\langle R_{m^{\prime}}\right\rangle$, deste modo, $\left\langle R_{m^{\prime}}\right\rangle$ contém uma base de $\left\langle S_{l(S)}\right\rangle=\mathcal{L}_{l(S)}(S)=\mathbb{O}$, concluindo que $R$ é gerador. Novamente, faremos por indução no comprimento das palavras irredutíveis.

Caso base: Tome $w \in S_{2}$ irredutível e de comprimento 2. Quando $w=x_{1} x_{j}$, por hipótese, podemos escrever $x_{1}$ como combinação linear de palavras cujo comprimento não excede $k$. Seja então $x_{1}=\left(\alpha_{k, 1} w_{k, 1}+\ldots+\alpha_{k, n_{k}} w_{k, n_{k}}\right)+\ldots+\left(\alpha_{1,1} w_{1,1}+\alpha_{1, n_{1}} w_{1, n_{1}}\right)+\alpha_{0} 1$, para alguns $\alpha_{i, j} \in \mathbb{R}$ e com $w_{i, j}$ representando a j-ésima palavra de comprimento $i$ que aparece na expressão de $x_{1}$. Concluímos que $x_{1} x_{j}=\left(\left(\alpha_{k, 1} w_{k, 1}+\ldots+\ldots+\alpha_{k, n_{k}} w_{k, n_{k}}\right)+\ldots+\left(\alpha_{1,1} w_{1,1}+\alpha_{1, n_{1}} w_{1, n_{1}}\right)+\alpha_{0} 1\right) x_{j}$. Como $x_{j} \in R$, podemos decompor $x_{1} x_{j}$ em uma combinação linear de palavras em $R$ cujo comprimento não excede o valor $k+1$, isto é, $x_{1} x_{j} \in\left\langle R_{k+1}\right\rangle$. Se $w=x_{i} x_{j} \operatorname{com} i, j \neq 1$ então $w \in R_{2}$ pela construção de $R$. Isso mostra que $S_{2} \subset\left\langle R_{k+1}\right\rangle$. 
Hipótese de Indução: Suponha que exista um índice $m$ tal que $S_{n-1} \subset\left\langle R_{m}\right\rangle$.

Passo indutivo: Seja $w \in S_{n}$ uma palavra irredutível de comprimento $n$. Pelo Lema 2.2, $w$ é o produto de outras duas palavras irredutíveis de comprimentos menores. Escrevemos $w=$ $w_{1} w_{2}$ e supomos que $l\left(w_{1}\right) \geq l\left(w_{2}\right)$. Por hipótese de indução, $w_{1} \in\left\langle R_{p_{1}}\right\rangle$ para algum inteiro positivo $p_{1}$. Consequentemente, $l\left(w_{1}\right) \geq l\left(w_{2}\right)$ implica em $w_{2} \in\left\langle R_{p_{1}}\right\rangle$. Podemos então expressar o produto $w=w_{1} w_{2}$ como o produto de combinações lineares de palavras irredutíveis de $R$ cujos comprimentos não excedem o valor $p_{1}$. Como esse produto deve pertencer a $\left\langle R_{2 p_{1}}\right\rangle$ concluímos que $w \in\left\langle R_{2 p_{1}}\right\rangle$.

Nosso objetivo é determinar o maior comprimento possível para um conjunto gerador, desse modo, chamaremos de conjunto gerador $P$-refinado um conjunto refinado que também está livre de elementos que satisfaçam as condições do Lema 5.7. Como observado anteriormente, para que $l(S)>3$ devemos ter que $5 \geq|S| \geq 3$, isto nos deixa em posição de demonstrar a proposição a seguir.

Proposição 5.5. Se $S \subset \mathrm{O}$ é um subconjunto gerador $P$-refinado e que satisfaz $|S|=5$, então $\operatorname{dim} \mathcal{L}_{2}(S)-\operatorname{dim} \mathcal{L}_{1}(S) \geq 2$

Demonstração. Seja $S=\left\{x_{1}, x_{2}, x_{3}, x_{4}, x_{5}\right\}$. Se $\operatorname{dim} \mathcal{L}_{2}(S)-\operatorname{dim} \mathcal{L}_{1}(S)=1$ podemos supor que $x_{1} x_{2}$ é uma palavra irredutível. Neste caso, qualquer palavra $w$ de comprimento dois pertence ao espaço gerado pelo conjunto $\left\{1, x_{1}, x_{2}, x_{3}, x_{4}, x_{5}, x_{1} x_{2}\right\}$ pela Proposição 5.4. Em particular, existem escalares $p_{i} \in \mathbb{R}$ tais que

$$
x_{3} x_{2}=p_{1}+p_{2} x_{1}+p_{3} x_{2}+p_{4} x_{3}+p_{5} x_{4}+p_{6} x_{5}+p_{7}\left(x_{1} x_{2}\right)
$$

Obtemos então a igualdade $x_{3} x_{2}-p_{3} x_{2}-p_{7}\left(x_{1} x_{2}\right)=p_{1}+p_{2} x_{1}+p_{4} x_{3}+p_{5} x_{4}+p_{6} x_{5}$, que pode ser reescrita como

$$
\left(x_{3}-p_{3}-p_{7} x_{1}\right) x_{2}=p_{1}+p_{2} x_{1}+p_{4} x_{3}+p_{5} x_{4}+p_{6} x_{5}
$$

De acordo com a Proposição 5.4 o elemento $\left(x_{3}-p_{3}-p_{7} x_{1}\right)^{-1}$ depende apenas de $1, x_{1}$ e $x_{3}$. Multiplicando ambos os lados da igualdade 5.3 à esquerda por $\left(x_{3}-p_{3}-p_{7} x_{1}\right)^{-1}$ concluímos que $x_{2}$ pode ser expresso como combinação linear de palavras que dependem apenas de $1, x_{1}, x_{3}, x_{4}, x_{5}$ cujos comprimentos não excedem o valor 2, isto é, $x_{2} \in\left\langle R_{2}\right\rangle$, com $R=S \backslash\left\{x_{2}\right\}$, contradizendo a hipótese de que $S$ é $P$-refinado.

Isto mostra que para qualquer conjunto gerador $P$-refinado $S$ satisfazendo $5 \geq|S| \geq 3$ temos que $\operatorname{dim} \mathcal{L}_{2}(S)-\operatorname{dim} \mathcal{L}_{1}(S) \geq 2$, em particular, se $|S|=5$ então $l(S) \leq 2$. Precisamos mostrar ainda que se $|S| \in\{3,4\}$ então também não pode ser que $l(S)>3$.

Proposição 5.6. Se $S \subset \mathbb{O}$ é um subconjunto gerador $P$-refinado e que satisfaz $4 \geq|S| \geq 3$. Então $\operatorname{dim} \mathcal{L}_{2}(S)-\operatorname{dim} \mathcal{L}_{1}(S) \geq 3$.

Demonstração. Podemos então supor que $\operatorname{dim} \mathcal{L}_{2}(S)-\operatorname{dim} \mathcal{L}_{1}(S)=2$ e que $|S|=4$, o caso em que $|S|=3$ pode ser tratado de modo similar. Apelando para uma reordenação dos elementos de $S$ se necessário, podemos supor que $x_{1} x_{2}$ é uma palavra irredutível e então o conjunto $\left\{1, x_{1}, x_{2}, x_{3}, x_{4}, x_{1} x_{2}\right\}$ é linearmente independente. Temos então os casos a seguir. 
Caso 1: A palavra que completa uma base para $\mathcal{L}_{2}(S)$ possui como um dos fatores o elemento $x_{1}$ ou o elemento $x_{2}$.

Observe que $x_{2} x_{1}$ não pode completar uma base pois nesse caso a álgebra gerada por $x_{1} \mathrm{e}$ $x_{2}$ seria associativa e com dimensão superior a 4 , contrariando o teorema de Frobenius.

Suponha que a palavra $x_{j} x_{1}, \operatorname{com} j \in\{3,4\}$ complete uma base, o caso $x_{j} x_{2}$ é análogo. Afirmamos que podemos substituir $x_{j} x_{1}$ por $x_{1} x_{j}$. Com efeito, a subálgebra gerada por $x_{j}$ e $x_{1}$ tem dimensão superior a três, é associativa, unitária e com divisão então deve ter dimensão 4 e consequentemente $\left\{1, x_{1}, x_{j}, x_{j} x_{1}\right\}$ é uma base para esta subálgebra. Podemos escrever $x_{1} x_{j}=q_{1}+q_{2} x_{1}+$ $q_{3} x_{j}+q_{4}\left(x_{j} x_{1}\right)$ com $q_{1}, q_{2}, q_{3}, q_{4}, q_{5} \in \mathbb{R}$. Se $q_{4}=0$ então $\left(x_{1}-q_{3}\right) x_{j}=q_{1}+q_{2} x_{1}$ e poderíamos ter eliminado $x_{j}$, contrariando o fato de $S$ ser $P$-refinado. Assim, se o conjunto $\left\{1, x_{1}, x_{2}, x_{3}, x_{4}, x_{1} x_{2}, x_{1} x_{j}\right\}$ é linearmente dependente, existem escalares $k_{i} \in \mathbb{R}$ tais que $x_{1} x_{j}=k_{1}+k_{2} x_{1}+k_{3} x_{2}+k_{4} x_{3}+k_{5} x_{4}+$ $k_{6}\left(x_{1} x_{2}\right)$. Como $q_{4} \neq 0$, concluímos que o conjunto $\left\{1, x_{1}, x_{2}, x_{3}, x_{4}, x_{1} x_{2}, x_{j} x_{1}\right\}$ também deve ser linearmente dependente, contrariando a hipótese de ser uma base de $\mathcal{L}_{2}(S)$.

Tomamos então a base de $\mathcal{L}_{2}(S)$ dada por $\left\{1, x_{1}, x_{2}, x_{3}, x_{4}, x_{1} x_{2}, x_{1} x_{j}\right\}$. Como $x_{4} x_{2} \in \mathcal{L}_{2}(S)$, existem escalares $r_{i} \in \mathbb{R}$ tais que $x_{1} x_{4}=r_{1}+r_{2} x_{1}+r_{3} x_{2}+r_{4} x_{3}+r_{5} x_{4}+r_{6}\left(x_{1} x_{2}\right)+r_{7}\left(x_{1} x_{j}\right)$ e então

$$
x_{1}\left(x_{4}-r_{2}-r_{6} x_{2}-r_{7} x_{j}\right)=r_{1}+r_{3} x_{2}+r_{4} x_{3}+r_{5} x_{4}
$$

Multiplicando à direita ambos os lados da igualdade 5.4 por $\left(x_{4}-r_{2}-r_{6} x_{2}-r_{7} x_{j}\right)^{-1}$ podemos concluir que $x_{1}$ pode ser escrito como combinação linear de palavras que utilizam apenas os elementos $1, x_{2}, x_{3}, x_{4}$ e cujos comprimentos não excedem o valor 2 , contrariando a hipótese de $S$ ser $P$-refinado. Então devemos ter $\operatorname{dim} \mathcal{L}_{2}(S)-\operatorname{dim} \mathcal{L}_{1}(S)>2$.

Caso 2: A palavra que completa uma base para $\mathcal{L}_{2}(S)$ não possui $x_{1}$ ou $x_{2}$ como fator, ou seja, é da forma $x_{3} x_{4}$ ou $x_{4} x_{3}$.

Podemos supor que $\left\{1, x_{1}, x_{2}, x_{3}, x_{4}, x_{1} x_{2}, x_{3} x_{4}\right\}$ é base de $\mathcal{L}_{2}(S)$. Como $x_{1} x_{4} \in \mathcal{L}_{2}(S)$, existem escalares $t_{i}$ tais que $x_{1} x_{4}=t_{1}+t_{2} x_{1}+t_{3} x_{2}+t_{4} x_{3}+t_{5} x_{4}+t_{6}\left(x_{1} x_{2}\right)+t_{7}\left(x_{3} x_{4}\right)$, concluímos então que

$$
x_{1}\left(x_{4}-t_{2}-t_{6} x_{2}\right)=t_{1}+t_{3} x_{2}+t_{4} x_{3}+t_{5} x_{4}+t_{7}\left(x_{3} x_{4}\right)
$$

Multiplicando à direita ambos os lados da igualdade (5.5) por $\left(x_{4}-t_{2}-t_{6} x_{2}\right)^{-1}$ concluímos que $x_{1}$ pode ser escrito como combinação linear de palavras que utilizam apenas os elementos $1, x_{2}, x_{3}, x_{4}$ contrariando a hipótese de que $S$ é $P$-refinado. Isso mostra que $\operatorname{dim} \mathcal{L}_{2}(S)-\operatorname{dim} \mathcal{L}_{1}(S)>2$.

Por fim, pelo que foi estabelecido nos Casos 1 e 2 , se $|S| \in\{3,4\}$ então $\operatorname{dim} \mathcal{L}_{2}(S)-\operatorname{dim} \mathcal{L}_{1}(S) \geq 3$, demonstrando a proposição.

Se $|S|=4$, a Proposição 5.6 estabelece que a cadeia de $S$ deve ser da forma $a_{0}=1 \rightarrow a_{1}=5 \rightarrow$ $a_{2}=5+3=8$ e teríamos que $l(S)<3$.

Vamos analisar o caso em que $|S|=3$. Pela Proposição 5.6 devemos ter uma cadeia na forma $a_{0}=1 \rightarrow a_{1}=4 \rightarrow a_{2}=7$, implicando em $a_{3}=7$ e $a_{4}=8$. Seja então $S=\left\{x_{1}, x_{2}, x_{3}\right\}$. Novamente, 
podemos supor que $x_{1} x_{2}$ é uma palavra irredutível em $S_{2}$, além disso, as palavras irredutíveis $w_{1}, w_{2}$ de comprimento dois que completam uma base para $\mathcal{L}_{2}(S)$ devem satisfazer as relações $w_{1} \in\left\{x_{1} x_{3}, x_{3} x_{1}\right\}$ e $w_{2} \in\left\{x_{2} x_{3}, x_{3} x_{2}\right\}$. Argumentando de modo similar ao Caso 1 da Proposição 5.6 podemos supor que $\left\{1, x_{1}, x_{2}, x_{3}, x_{1} x_{2}, x_{1} x_{3}, x_{2} x_{3}\right\}$ é uma base de $\mathcal{L}_{2}(S)$.

Se $w$ é uma palavra irredutível de comprimento 4, então deve ser produto de outras duas palavras irredutíveis de comprimento 2. Vamos mostrar que $\left(x_{1} x_{2}\right)\left(x_{1} x_{3}\right)$ é irredutível se, e somente se, $\left(x_{1} x_{2}\right)\left(x_{3} x_{1}\right)$ é irredutível. Pelo Teorema de Frobenius, sabemos que existem escalares $p_{i}$ tais que $x_{3} x_{1}=p_{1}+p_{2} x_{1}+p_{3} x_{3}+p_{4}\left(x_{1} x_{3}\right)$. Além disso, é possível considerar $p_{4} \neq 0$, pois caso contrário poderíamos utilizar o Lema 5.7 para remover $x_{1}$ e então $S$ não seria $P$-refinado. Obtemos então as igualdades

$$
\begin{gathered}
\left(x_{1} x_{2}\right)\left(x_{3} x_{1}\right)=\left(x_{1} x_{2}\right)\left(p_{1}+p_{2} x_{1}+p_{3} x_{3}+p_{4}\left(x_{1} x_{3}\right)\right)= \\
p_{1}\left(x_{1} x_{2}\right)+p_{2}\left(\left(x_{1} x_{2}\right) x_{1}\right)+p_{3}\left(\left(x_{1} x_{2}\right) x_{3}\right)+p_{4}\left(\left(x_{1} x_{2}\right)\left(x_{1} x_{3}\right)\right)
\end{gathered}
$$

que podem ser reescritas como

$$
\left(x_{1} x_{2}\right)\left(x_{3} x_{1}\right)-p_{4}\left(x_{1} x_{2}\right)\left(x_{1} x_{3}\right)=p_{1}\left(x_{1} x_{2}\right)+p_{2}\left(\left(x_{1} x_{2}\right) x_{1}\right)+p_{3}\left(\left(x_{1} x_{2}\right) x_{3}\right)
$$

Quando o lado esquerdo da igualdade (5.6) é zero, o resultado está provado pois um múltiplo escalar não nulo de um vetor linearmente independente é ainda um vetor linearmente independente. Suponha então que $\left(x_{1} x_{2}\right)\left(x_{3} x_{1}\right)-p_{4}\left(x_{1} x_{2}\right)\left(x_{1} x_{3}\right) \neq 0$. Segue da igualdade $\mathcal{L}_{2}(S)=\mathcal{L}_{3}(S)$ que o lado direito da equação 5.6 pertence a $\mathcal{L}_{2}(S)$. Consequentemente, $\left(x_{1} x_{2}\right)\left(x_{3} x_{1}\right) \in \mathcal{L}_{2}(S)$ se, e somente se, $\left(x_{1} x_{2}\right)\left(x_{1} x_{3}\right) \in \mathcal{L}_{2}(S)$. Isto mostra que podemos permutar os pares de elementos entre parênteses sem que isso torne a palavra redutível. Aplicamos este raciocínio e concluímos as seguintes relações.

- $\left(x_{1} x_{2}\right)\left(x_{2} x_{3}\right)$ é irredutível se, e somente se, $\left(x_{2} x_{1}\right)\left(x_{2} x_{3}\right)$ é irredutível se, e somente se, $\left(x_{2} x_{1}\right)\left(x_{3} x_{2}\right)$ é irredutível;

- $\left(x_{1} x_{2}\right)\left(x_{1} x_{3}\right)$ é irredutível se, e somente se, $\left(x_{1} x_{2}\right)\left(x_{3} x_{1}\right)$ é irredutível;

- $\left(x_{2} x_{3}\right)\left(x_{1} x_{2}\right)$ é irredutível se, e somente se, $\left(x_{2} x_{3}\right)\left(x_{2} x_{1}\right)$ é irredutível;

- $\left(x_{1} x_{3}\right)\left(x_{1} x_{2}\right)$ é irredutível se, e somente se, $\left(x_{1} x_{3}\right)\left(x_{2} x_{1}\right)$ é irredutível;

- $\left(x_{2} x_{3}\right)\left(x_{1} x_{3}\right)$ é irredutível se, e somente se, $\left(x_{3} x_{2}\right)\left(x_{1} x_{3}\right)$ é irredutível;

- $\left(x_{1} x_{3}\right)\left(x_{2} x_{3}\right)$ é irredutível se, e somente se, $\left(x_{3} x_{1}\right)\left(x_{2} x_{3}\right)$ é irredutível.

Provaremos que nenhuma palavra na forma $\left(x_{k} x_{j}\right)\left(x_{i} x_{k}\right)$ com $x_{i} \neq x_{j} \neq x_{k} \neq x_{i}$ pode ser irredutível e como consequência das observações acima poderemos concluir que não existe uma palavra irredutível de comprimento 4 , consequentemente $\operatorname{dim} \mathcal{L}_{3}(S)>\operatorname{dim} \mathcal{L}_{2}(S)=a_{2}=7$ e então $l(S) \leq 3$. Pela identidade de Moufang ao meio temos $\left(x_{k} x_{j}\right)\left(x_{i} x_{k}\right)=\left(x_{k}\left(x_{j} x_{i}\right)\right) x_{k}$. Segue da igualdade $\mathcal{L}_{2}(S)=\mathcal{L}_{3}(S)$ que $x_{k}\left(x_{j} x_{i}\right) \in\left\langle 1, x_{1}, x_{2}, x_{3}, x_{1} x_{2}, x_{1} x_{3}, x_{2} x_{3}\right\rangle$ e consequentemente $\left(x_{k}\left(x_{j} x_{i}\right)\right) x_{k} \in \mathcal{L}_{3}(S)=\mathcal{L}_{2}(S)$. Isso mostra que a cadeia $1 \rightarrow 4 \rightarrow 7 \rightarrow 7 \rightarrow 8$ é impossível, consequentemente, se $|S|=3$ então $l(S) \leq 3$. Essa discussão prova a proposição.

Proposição 5.7. Seja $S \subset \mathrm{O}$ um subconjunto gerador $P$-refinado de três elementos. Então $l(S) \leq 3$. 
Corolário 5.2. O comprimento da $\mathbb{R}$-álgebra dos Octônios é três.

Prosseguimos da seguinte forma. Se $S$ é um subconjunto gerador, podemos tomar seu refinamento para obter um conjunto de igual ou maior comprimento mas com a propriedade de ser $P$-refinado. Deste modo, limitamos o comprimento de um conjunto gerador $S$ de cardinalidade maior que 3 por $l(S) \leq 2$. Para conjuntos geradores de cardinalidade 3 , aplicamos o mesmo processo e pela Proposição 5.7, o maior comprimento que este pode atingir é 3 . O conjunto $S=\{i, j, l\}$ tem comprimento 3. Com efeito, $\mathcal{L}_{0}(S)=\langle 1\rangle, \mathcal{L}_{1}(S)=\langle 1, i, j, l\rangle, \mathcal{L}_{2}(S)=\langle 1, i, j, l, k, i l, j l\rangle$, $\mathcal{L}_{3}(S)=\langle 1, i, j, l, k, i l, j l, k l\rangle=\mathrm{O}$, provando o resultado. 


\section{Apêndice A}

\section{Programas Auxiliares em linguagem C}

Para auxiliar nas verificações e conjecturar resultados fizemos uso de métodos computacionais. Para entender como o algoritimo foi construído, fixamos a álgebra 5.1 e denotamos $x=x_{1} e+x_{2} a+$ $x_{3} b+x_{4} c+x_{5} d$ por $x=\left(x_{1}, x_{2}, x_{3}, x_{4}, x_{5}\right)$; e $y=y_{1} e+y_{2} a+y_{3} b+y_{4} c+y_{5} d$ por $y=\left(y_{1}, y_{2}, y_{3}, y_{4}, y_{5}\right)$. Observando agora que $x y=a_{1} y+\left(a_{2} b_{1}-a_{1} b_{2}+a_{3} b_{4}-a_{4} b_{3}\right) a+\left(a_{5} b_{1}\right) d$ retornamos à notação vetorial somando coordenada à coordenada. Definimos então as variáveis auxiliares $r_{i}$ onde $r_{i}$ será a $i$ ésima coordenada do produto $x y$, por exemplo $r_{2}=x_{1} y_{2}+x_{2} y_{1}-x_{1} y_{2}+x_{3} y_{4}-x_{4} y_{3}$ e o programa retorna o valor do produto em notação vetorial. Abaixo exemplificamos o resultado da aplicação do método. Ressaltamos que pela Proposição 5.1 não é necessário encontrar um algoritmo que calcule o produto nas álgebras opostas pois o comprimento destas álgebras coincidem. 


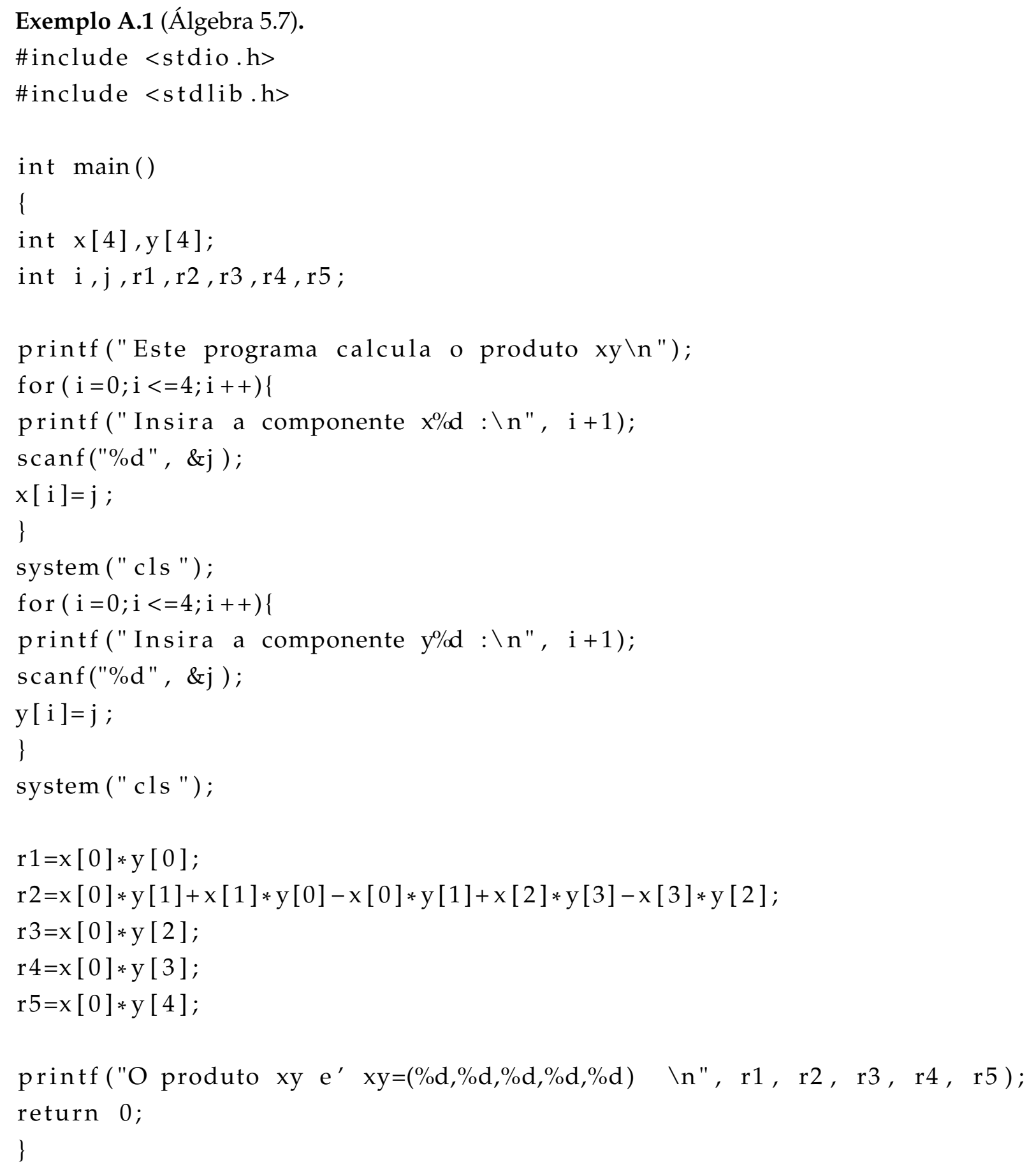




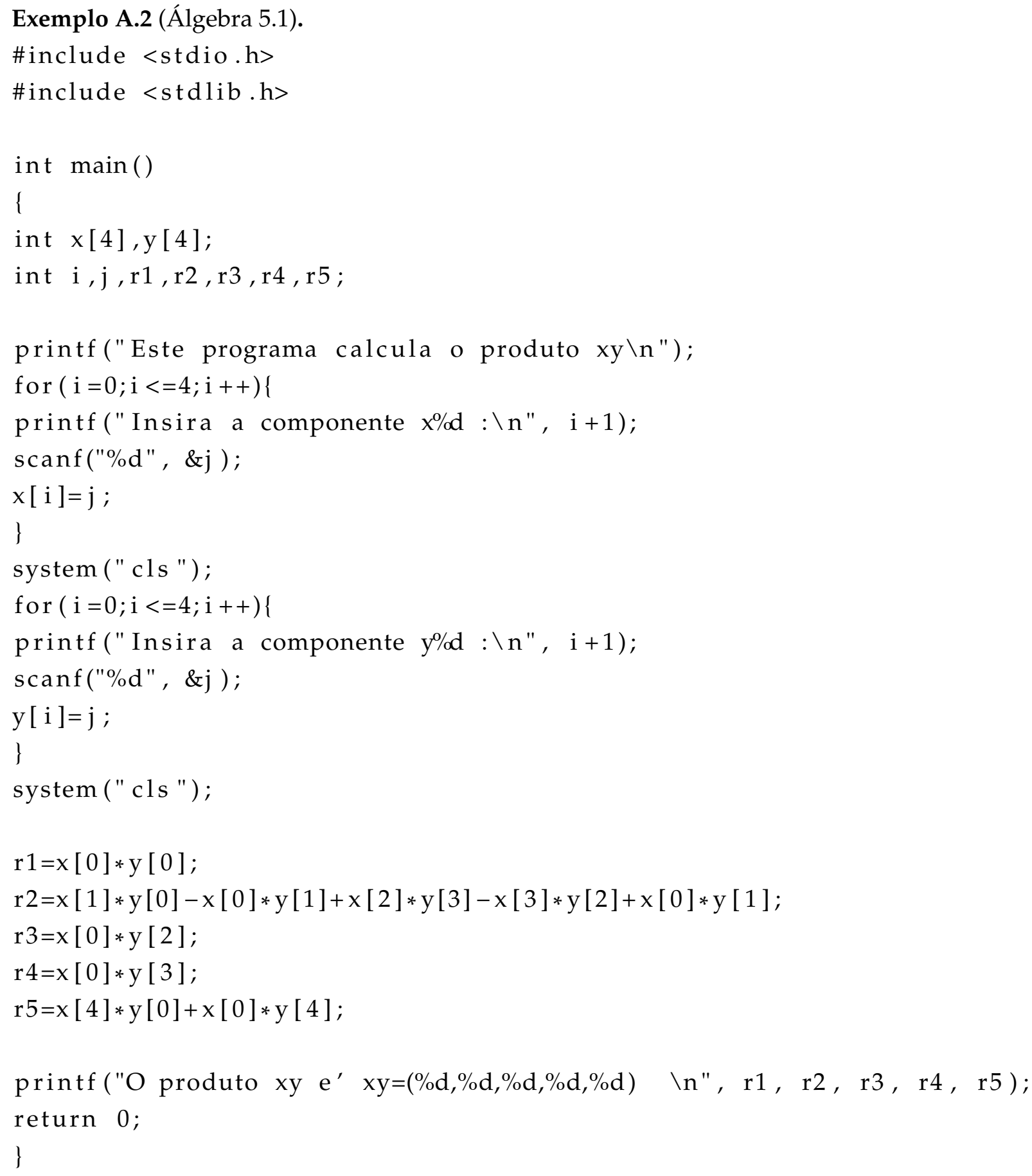


Exemplo A.3 (Álgebra 5.3).

\#include <stdio.h>

\#include <stdlib.h>

int main ()

\{

int $x[4], y[4]$;

int $\mathrm{i}, \mathrm{j}, \mathrm{r} 1, \mathrm{r} 2, \mathrm{r} 3, \mathrm{r} 4, \mathrm{r} 5$;

printf("Este programa calcula o produto $x y \backslash n ")$;

for $(\mathrm{i}=0 ; \mathrm{i}<=4 ; \mathrm{i}++)\{$

printf("Insira a componente $\mathrm{x} \% \mathrm{~d}: \backslash \mathrm{n} ", \mathrm{i}+1)$;

$\operatorname{scanf}(" \% d ", \& j)$;

$x[i]=j$;

\}

system (" cls ");

for $(\mathrm{i}=0 ; \mathrm{i}<=4 ; \mathrm{i}++)\{$

printf("Insira a componente $\mathrm{y} \% \mathrm{~d}: \backslash \mathrm{n} ", \mathrm{i}+1)$;

$\operatorname{scanf}(" \% d ", \& j)$;

$y[i]=j$;

\}

system ("cls");

$\mathrm{r} 1=\mathrm{x}[0] * \mathrm{y}[0]$;

$\mathrm{r} 2=\mathrm{x}[1] * \mathrm{y}[0]-\mathrm{x}[0] * \mathrm{y}[1]+\mathrm{x}[2] * \mathrm{y}[3]-\mathrm{x}[3] * \mathrm{y}[2]+\mathrm{x}[0] * \mathrm{y}[1] ;$

$\mathrm{r} 3=\mathrm{x}[0] \mathrm{y}[2]$;

$\mathrm{r} 4=\mathrm{x}[0] \mathrm{y}[3]$;

printf ("O produto $\left.x y e^{\prime} x y=(\% d, \% d, \% d, \% d, 0) \quad \backslash n ", r 1, r 2, \quad r 3, \quad r 4\right)$;

return 0 ;

\} 
Exemplo A.4 (Álgebra 5.5).

\#include <stdio.h>

\#include <stdlib.h>

int $\operatorname{main}()$

\{

int $\mathrm{x}[4], \mathrm{y}[4]$;

in $\mathrm{t} i, \mathrm{j}, \mathrm{r} 1, \mathrm{r} 2, \mathrm{r} 3, \mathrm{r} 4, \mathrm{r} 5$;

printf("Este programa calcula o produto $x y \backslash n ")$;

for $(\mathrm{i}=0 ; \mathrm{i}<=4 ; \mathrm{i}++)\{$

printf("Insira a componente $\mathrm{x} \% \mathrm{~d}: \backslash \mathrm{n} ", \quad \mathrm{i}+1)$;

scanf $(" \% d ", \& j)$;

$x[i]=j$;

\}

system (" cls ");

for $(\mathrm{i}=0 ; \mathrm{i}<=4 ; \mathrm{i}++)\{$

printf("Insira a componente $\mathrm{y} \% \mathrm{~d}: \backslash \mathrm{n} ", \quad \mathrm{i}+1)$;

$\operatorname{scanf}(" \% d ", \& j)$;

$y[i]=j$;

\}

system (" cls ");

$\mathrm{r} 1=\mathrm{x}[0] \mathrm{y}[0] ;$

$\mathrm{r} 2=\mathrm{x}[0] \mathrm{y}[1]+\mathrm{x}[1] \mathrm{y}[0]+\mathrm{x}[2] \mathrm{y}[3]-\mathrm{x}[3] \mathrm{y}[2]-\mathrm{x}[0] \mathrm{y}[1]$;

$\mathrm{r} 3=\mathrm{x}[0] \mathrm{y}[2]$;

$\mathrm{r} 4=\mathrm{x}[0] \mathrm{y}[3]$;

r5 $=x[4]$ y [0] ;

printf ("O produto $\left.x y e^{\prime} x y=(\% d, \% d, \% d, \% d, \% d) \quad \backslash n ", r 1, r 2, r 3, r 4, r 5\right)$; return 0 ;

\} 


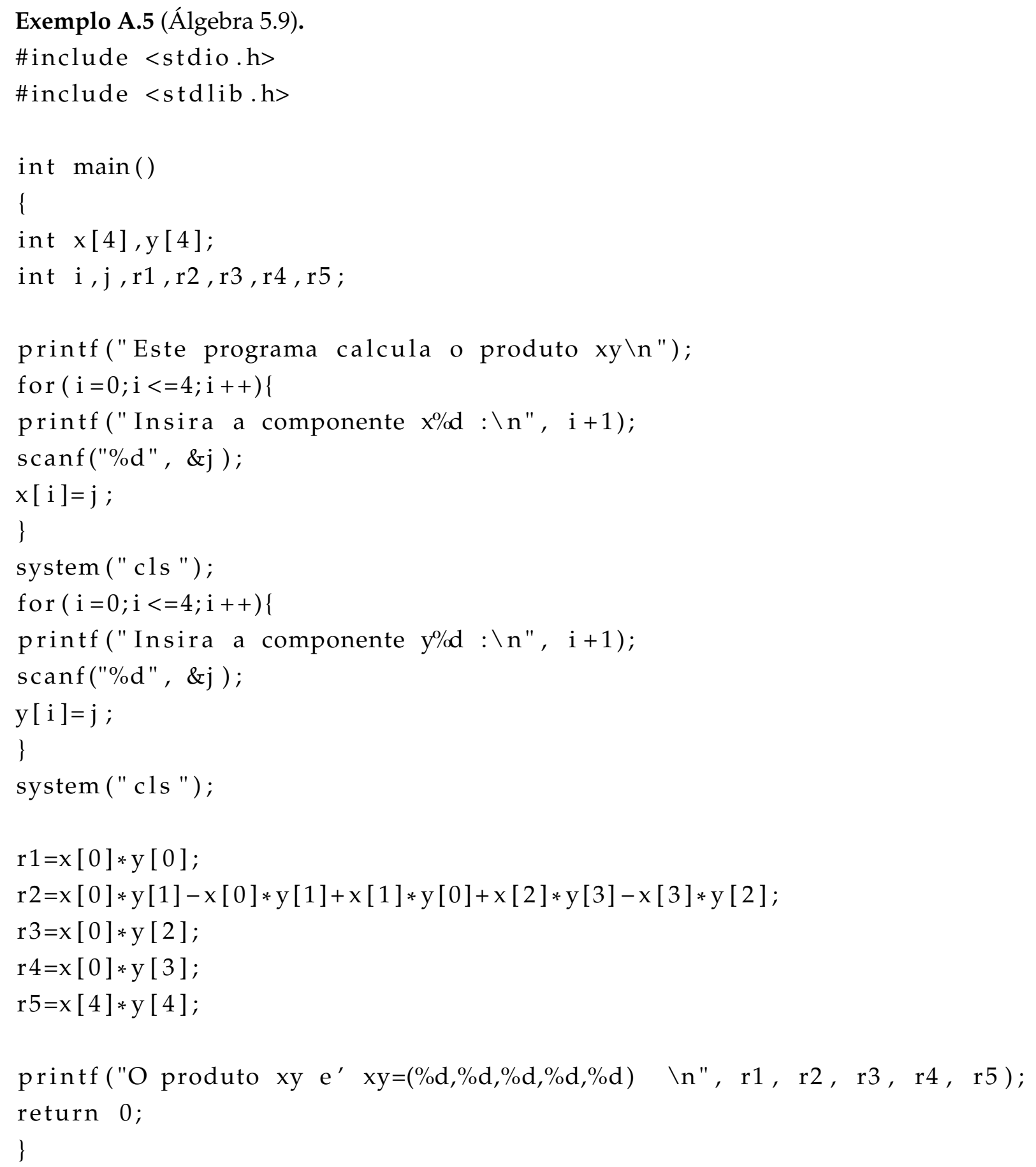


Exemplo A.6 (Álgebra 5.0).

\#include <stdio.h>

\#include <stdlib.h>

int $\operatorname{main}()$

\{

int $\mathrm{x}[4], \mathrm{y}[4]$;

int $\mathrm{i}, \mathrm{j}, \mathrm{r} 1, \mathrm{r} 2, \mathrm{r} 3, \mathrm{r} 4, \mathrm{r} 5$;

printf("Este programa calcula o produto $x y \backslash n ")$;

for $(\mathrm{i}=0 ; \mathrm{i}<=4 ; \mathrm{i}++)\{$

printf("Insira a componente $\mathrm{x} \% \mathrm{~d}: \backslash \mathrm{n} ", \quad \mathrm{i}+1)$;

scanf $(" \% d ", \& j)$;

$x[i]=j$;

\}

system (" cls ");

for $(\mathrm{i}=0 ; \mathrm{i}<=4 ; \mathrm{i}++)\{$

printf("Insira a componente $\mathrm{y} \% \mathrm{~d}: \backslash \mathrm{n} ", \mathrm{i}+1)$;

$\operatorname{scanf}(" \% d ", \& j)$;

$\mathrm{y}[\mathrm{i}]=\mathrm{j}$;

\}

system (" cls ");

$\mathrm{r} 1=\mathrm{y}[0] * \mathrm{x}[0] ;$

$\mathrm{r} 2=\mathrm{y}[1] * \mathrm{x}[0]+\mathrm{y}[0] * \mathrm{x}[1]+\mathrm{x}[3] * \mathrm{y}[1]+\mathrm{x}[1] * \mathrm{y}[1] ;$

$\mathrm{r} 3=\mathrm{x}[0] * \mathrm{y}[2]+\mathrm{x}[2] * \mathrm{y}[0]+\mathrm{x}[1] * \mathrm{y}[2]$;

$\mathrm{r} 4=\mathrm{y}[0] * \mathrm{x}[3]+\mathrm{x}[0] * \mathrm{y}[3]$;

$\mathrm{r} 5=\mathrm{x}[0] * \mathrm{y}[4]+\mathrm{x}[4] * \mathrm{y}[0]+\mathrm{x}[4] * \mathrm{y}[1]$;

printf ("O produto $\left.x y e^{\prime} x y=(\% d, \% d, \% d, \% d, \% d) \backslash n ", r 1, r 2, r 3, r 4, r 5\right)$; return 0 ;

\} 


\section{Referências Bibliográficas}

[1] A. Albert. Structure of Algebras. American Mathematical Society, 1980.

[2] M. Badalov. Nilpotent alternative algebras. Algebra i Logik, 23:167-181, 1984.

[3] A. Cayley. XXVII. on Jacobi's Elliptic functions, in reply to the Rev. Brice Bronwin; and on Quaternions: To the editos of the Philosophical magazine and Journal, 1845.

[4] A. Gainov. Alternative algebras of rank 3 and 4. Algebra i Logik, 4:41-46, 1963.

[5] M. Gerstenhaber. On dominance and varieties of commuting matrices. Annals of Mathematics, 73(2):324-348, 1961.

[6] E. Goodaire and Y. Zhou. Alternative rings of small order. Communications in Algebra, 28(7):3335-3349, 2000.

[7] A. Guterman and D. Kudryavtsev. Upper bounds for the length of non-associative algebras. Journal of Algebra, 544:483 - 497, 2020.

[8] A. Guterman, T. Laffey, O. Markova, and H. Smigoc. A resolution of Paz's conjecture in the presence of a nonderogatory matrix. Linear Algebra and its Applications, 543:234 - 250, 2018.

[9] A. Guterman and O. Markova. Commutative matrix subalgebras and length function. Linear Algebra and its Applications, 430(7):1790 - 1805, 2009.

[10] A. Guterman, O. Markova, and V. Mehrmann. Length realizability for pairs of quasicommuting matrices. Linear Algebra and its Applications, 568:135-154, 2019.

[11] W. Hamilton. Note, by Sir. W. R. Hamilton, respecting the researches of John T. Graves, Esq. Transactions of the Royal Irish Academy, 21:338-341, 1848.

[12] E. Kuzmin. Maltsev algebras of dimension five over a field of characteristic zero. Algebra $i$ Logika, 9:691-700, 1970.

[13] O. Markova. Upper bound for the length of commutative algebras. Sbornik: Mathematics, 200(12):1767-1787, 2009.

[14] O. Markova. On some properties of the length function. Mathematical Notes, 87:71-78, 2010.

[15] P. Moritz. Basic Algebra: Groups, Rings and Fields. Springer, 2002.

[16] V. Morozov. The classification of nilpotent lie algebras of order six. Izvestiya Vysshikh Uchebnykh Zavedenii. Mat., 4:161-171, 1958. 
[17] V. Olshevsky and E. Tyrtyshnikov. Matrix Methods: Theory, Algorithms and Applications. World Scientific, year $=2010$,

[18] R. Palais. The classification of real division algebras. American Mathematical Monthly, 75:366$368,1968$.

[19] C. Pappacena. An upper bound for the length of a finite-dimensional algebra. Journal of Algebra, 197(2):535-545, 1997.

[20] A. Paz. An application of the Cayley-Hamilton theorem to matrix polynomials in several variables. Linear and Multilinear Algebra, 15(2):161-170, 1984.

[21] B. Peirce. Linear associative algebra. American Journal of Mathematics, 4(1):97-229, 1881.

[22] R. Schafer. An introduction to nonassociative algebras. Academic Press, 1966.

[23] I. Schur. Zur theorie der vertauschbaren matrizen. Journal für die reine und angewandte Mathematik, 130:66-76, 1905.

[24] Y. Shitov. An improved bound for the lengths of matrix algebras. Algebra Number Theory, 13:1501-1507, 2019.

[25] A. Spencer and R. Rivlin. The theory of matrix polynomials and its applications to the mechanics of isotropic continua. Archive for Rational Mechanics Analysis., 2:309-336, 1959.

[26] K. Zhevlakov, A. Slin'ko, I. Shestakov, and A. Shirshov. Rings that are nearly associative. Academic Press, Inc, 1982. 